\title{
The On-orbit calibration of the Fermi Large Area Telescope
}

\author{
The Fermi LAT Collaboration
}
A. A. Abdo ${ }^{1,2}$, M. Ackermann ${ }^{3}$, M. Ajello ${ }^{3}$, J. Ampe ${ }^{2}$, B. Anderson ${ }^{4}$, W. B. Atwood ${ }^{4}$, M. Axelsson ${ }^{5,6}$, R. Bagagli ${ }^{7}$, L. Baldini ${ }^{7}$, J. Ballet ${ }^{8}$, G. Barbiellini ${ }^{9,10}$, J. Bartelt ${ }^{3}$, D. Bastieri ${ }^{11,12}$, B. M. Baughman ${ }^{13}$, K. Bechtol ${ }^{3}$, D. Bédérède ${ }^{14}$, F. Bellardi ${ }^{7}$, R. Bellazzinii ${ }^{7}$, F. Belli ${ }^{15,16}$, B. Berenji ${ }^{3}$, D. Bisello ${ }^{11,12}$, E. Bissaldi ${ }^{17}$, E. D. Bloom ${ }^{3}$, G. Bogaert ${ }^{18}$, J. R. Bogart ${ }^{3}$, E. Bonamente $^{19,20}$, A. W. Borgland ${ }^{3}$, P. Bourgeois ${ }^{14}$, A. Bouvier ${ }^{3}$, J. Bregeon ${ }^{7}$, A. Brez ${ }^{7}$, M. Brigida ${ }^{21,22}$, P. Bruel ${ }^{18}$, T. H. Burnett ${ }^{23}$, G. Busetto ${ }^{11,12}$, G. A. Caliandro ${ }^{21,22}$, R. A. Cameron ${ }^{3}$, M. Campell ${ }^{3}$, P. A. Caraveo ${ }^{24}$, S. Carius ${ }^{25}$, P. Carlson ${ }^{5,26}$, J. M. Casandjian ${ }^{8}$, E. Cavazzuti ${ }^{27}$, M. Ceccanti ${ }^{7}$, C. Cecchi ${ }^{19,20}$, E. Charles ${ }^{3}$, A. Chekhtman ${ }^{28,2}$, C. C. Cheung ${ }^{29}$, J. Chiang ${ }^{3}$, R. Chipaux ${ }^{30}$, A. N. Cillis ${ }^{29}$, S. Ciprini ${ }^{19,20}$, R. Claus $^{3}$, J. Cohen-Tanugi ${ }^{31}$, S. Condamoor ${ }^{3}$, J. Conrad ${ }^{5,26,32}$, R. Corbet $^{29}$, S. Cutini ${ }^{27}$, D. S. Davis ${ }^{29,33}$, M. DeKlotz ${ }^{34}$, C. D. Dermer ${ }^{2}$, A. de Angelis ${ }^{35}$, F. de Palma ${ }^{21,22}$, S. W. Digel ${ }^{3}$, P. Dizon ${ }^{36}$, M. Dormody ${ }^{4}$, E. do Couto e Silva ${ }^{3}$, P. S. Drell ${ }^{3}$, R. Dubois ${ }^{3}$, D. Dumora ${ }^{37,38}$, Y. Edmonds ${ }^{3}$, D. Fabiani ${ }^{7}$, C. Farnier ${ }^{31}$, C. Favuzzi ${ }^{21,22}$, E. C. Ferrara ${ }^{29}$, O. Ferreira ${ }^{18}$, Z. Fewtrell ${ }^{2}$, D. L. Flath ${ }^{3}$, P. Fleury ${ }^{18}$, W. B. Focke ${ }^{3}$, K. Fouts ${ }^{3}$, M. Frailis ${ }^{35}$, D. Freytag 3 , Y. Fukazawa ${ }^{39}$, S. Funk ${ }^{3}$, P. Fusco ${ }^{21,22}$, F. Gargano ${ }^{22}$, D. Gasparrini ${ }^{27}$, N. Gehrels ${ }^{29,40}$, S. Germani ${ }^{19,20}$, B. Giebels ${ }^{18}$, N. Giglietto ${ }^{21,22}$, F. Giordano ${ }^{21,22}$, T. Glanzman ${ }^{3}$, G. Godfrey ${ }^{3}$, J. Goodman ${ }^{3}$, I. A. Grenier ${ }^{8}$, M.-H. Grondin ${ }^{37,38}$, J. E. Grove ${ }^{2}$, L. Guillemot ${ }^{37,38}$, S. Guiriec ${ }^{31}$, M. Hakimi ${ }^{3}$, G. Haller ${ }^{3}$, Y. Hanabata ${ }^{39}$, P. A. Hart ${ }^{3}$, P. Hascall ${ }^{41}$, E. Hays ${ }^{29}$, M. Huffer ${ }^{3}$, R. E. Hughes ${ }^{13}$, G. Jóhannesson ${ }^{3}$, A. S. Johnson ${ }^{3}$, R. P. Johnson ${ }^{4}$, T. J. Johnson ${ }^{29,40}$, W. N. Johnson ${ }^{2}$, T. Kamae ${ }^{3}$, H. Katagiri ${ }^{39}$, J. Kataoka ${ }^{42}$, A. Kavelaars ${ }^{3}$, H. Kelly ${ }^{3}$, M. Kerr ${ }^{23}$, W. Klamra ${ }^{5,26}$, J. Knödlseder ${ }^{43}$, M. L. Kocian ${ }^{3}$, F. Kuehn ${ }^{13}$, M. Kuss ${ }^{7}$, L. Latronico ${ }^{7}$, C. Lavalley $^{31}$, B. Leas ${ }^{2}$, B. Lee ${ }^{41}$, S.-H. Lee ${ }^{3}$, M. Lemoine-Goumard ${ }^{37,38}$, F. Longo,10, F. Loparco ${ }^{21,22}$, B. Lott ${ }^{37,38}$, M. N. Lovellette ${ }^{2}$, P. Lubrano ${ }^{19,20}$, D. K. Lung ${ }^{41}$, G. M. Madejski ${ }^{3}$, A. Makeev ${ }^{28,2}$, B. Marangelli ${ }^{21,22}$, M. Marchetti ${ }^{15,16}$, M. M. Massai ${ }^{7}$, D. May², G. Mazzenga ${ }^{15,16}$, M. N. Mazziotta ${ }^{22}$, J. E. McEnery ${ }^{29}$, S. McGlynn ${ }^{5,26}$, C. Meurer ${ }^{5,32}$, P. F. Michelson ${ }^{3}$, M. Minuti ${ }^{7}$, N. Mirizzi21,22, P. Mitra ${ }^{3}$, W. Mitthumsiri ${ }^{3}$, T. Mizuno ${ }^{39}$, A. A. Moiseev ${ }^{44}$, M. Mongelli ${ }^{22}$, C. Monte ${ }^{21,22}$, M. E. Monzani ${ }^{3}$, E. Moretti ${ }^{9,10}$, A. Morselli ${ }^{15}$, I. V. Moskalenko ${ }^{3}$, S. Murgia ${ }^{3}$, D. Nelson ${ }^{3}$, L. Nilsson ${ }^{25,45}$, S. Nishino ${ }^{39}$, P. L. Nolan ${ }^{3}$, E. Nuss ${ }^{31}$, M. Ohno ${ }^{46}$, T. Ohsugi ${ }^{39}$, N. Omodei ${ }^{7}$, E. Orlando ${ }^{17}$, J. F. Ormes ${ }^{47}$, M. Ozaki ${ }^{46}$, A. Paccagnella ${ }^{11,48}$, D. Paneque ${ }^{3}$, J. H. Panetta ${ }^{3}$, D. Parent ${ }^{37,38}$, V. Pelassa ${ }^{31}$, M. Pepe ${ }^{19,20}$, M. Pesce-Rollins ${ }^{7}$, P. Picozza ${ }^{15,16}$, M. Pinchera ${ }^{7}$, F. Piron ${ }^{31}$, T. A. Porter ${ }^{4}$, S. Rainò ${ }^{21,22}$, R. Rando ${ }^{11,12}$, E. Rapposelli ${ }^{7}$, W. Raynor ${ }^{2}$, M. Razzano ${ }^{7}$, A. Reimer ${ }^{3}$, O. Reimer ${ }^{3}$, T. Reposeur ${ }^{37,38}$, L. C. Reyes ${ }^{49}$, S. Ritz ${ }^{29,40}$, S. Robinson ${ }^{50,23}$, L. S. Rochester ${ }^{3}$, A. Y. Rodriguez ${ }^{51}$, R. W. Romani ${ }^{3}$, M. Roth $^{23}$, F. Ryde ${ }^{5,26}$, A. Sacchetti2 ${ }^{22}$, H. F.-W. Sadrozinski ${ }^{4}$, N. Saggini ${ }^{7}$, D. Sanchez ${ }^{18}$, L. Sapozhnikov ${ }^{3}$, O. H. Saxton ${ }^{3}$, P. M. Saz Parkinson ${ }^{4}$, A. Sellerholm ${ }^{5,32}$, C. Sgrò ${ }^{7}$, E. J. Siskind ${ }^{52}$, D. A. Smith ${ }^{37,38}$, P. D. Smith ${ }^{13}$, G. Spandre ${ }^{7}$, P. Spinelli ${ }^{21,22}$, J.-L. Starck ${ }^{8}$, T. E. Stephens ${ }^{29}$, M. S. Strickman ${ }^{2}$, A. W. Strong ${ }^{17}$, M. Sugizaki ${ }^{3}$, D. J. Suson ${ }^{53}$, H. Tajima ${ }^{3}$, H. Takahashi ${ }^{39}$, T. Takahashii6 ${ }^{46}$ T. Tanaka ${ }^{3}$, A. Tenze ${ }^{7}$, J. B. Thayer ${ }^{3}$, J. G. Thayer ${ }^{3}$, D. J. Thompson ${ }^{29}$, L. Tibaldo ${ }^{11,12}$, O. Tibolla ${ }^{54}$,

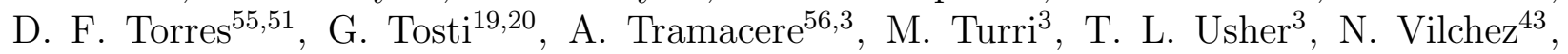


N. Virmani ${ }^{36}$, V. Vitale ${ }^{15,16}$, L. L. Wai ${ }^{3,57}$, A. P. Waite ${ }^{3}$, P. Wang ${ }^{3}$, B. L. Winer ${ }^{13}$, D. L. Wood ${ }^{2}$, K. S. Wood ${ }^{2}$, H. Yasuda ${ }^{39}$, T. Ylinen ${ }^{25,5,26}$, M. Ziegler ${ }^{4}$

1. National Research Council Research Associate

2. Space Science Division, Naval Research Laboratory, Washington, DC 20375

3. W. W. Hansen Experimental Physics Laboratory, Kavli Institute for Particle Astrophysics and Cosmology, Department of Physics and SLAC National Laboratory, Stanford University, Stanford, CA 94305

4. Santa Cruz Institute for Particle Physics, Department of Physics and Department of Astronomy and Astrophysics, University of California at Santa Cruz, Santa Cruz, CA 95064

5. The Oskar Klein Centre for Cosmo Particle Physics, AlbaNova, SE-106 91 Stockholm, Sweden

6. Department of Astronomy, Stockholm University, SE-106 91 Stockholm, Sweden

7. Istituto Nazionale di Fisica Nucleare, Sezione di Pisa, I-56127 Pisa, Italy

8. Laboratoire AIM, CEA-IRFU/CNRS/Université Paris Diderot, Service d'Astrophysique, CEA Saclay, 91191 Gif sur Yvette, France

9. Istituto Nazionale di Fisica Nucleare, Sezione di Trieste, I-34127 Trieste, Italy

10. Dipartimento di Fisica, Università di Trieste, I-34127 Trieste, Italy

11. Istituto Nazionale di Fisica Nucleare, Sezione di Padova, I-35131 Padova, Italy

12. Dipartimento di Fisica "G. Galilei”, Università di Padova, I-35131 Padova, Italy

13. Department of Physics, Center for Cosmology and Astro-Particle Physics, The Ohio State University, Columbus, OH 43210

14. IRFU/Dir, CEA Saclay, 91191 Gif sur Yvette, France

15. Istituto Nazionale di Fisica Nucleare, Sezione di Roma "Tor Vergata", I-00133 Roma, Italy

16. Dipartimento di Fisica, Università di Roma "Tor Vergata", I-00133 Roma, Italy

17. Max-Planck Institut für extraterrestrische Physik, 85748 Garching, Germany

18. Laboratoire Leprince-Ringuet, École polytechnique, CNRS/IN2P3, Palaiseau, France

19. Istituto Nazionale di Fisica Nucleare, Sezione di Perugia, I-06123 Perugia, Italy

20. Dipartimento di Fisica, Università degli Studi di Perugia, I-06123 Perugia, Italy 
21. Dipartimento di Fisica "M. Merlin" dell'Università e del Politecnico di Bari, I-70126 Bari, Italy

22. Istituto Nazionale di Fisica Nucleare, Sezione di Bari, 70126 Bari, Italy

23. Department of Physics, University of Washington, Seattle, WA 98195-1560

24. INAF-Istituto di Astrofisica Spaziale e Fisica Cosmica, I-20133 Milano, Italy

25. School of Pure and Applied Natural Sciences, University of Kalmar, SE-391 82 Kalmar, Sweden

26. Department of Physics, Royal Institute of Technology (KTH), AlbaNova, SE-106 91 Stockholm, Sweden

27. Agenzia Spaziale Italiana (ASI) Science Data Center, I-00044 Frascati (Roma), Italy

28. George Mason University, Fairfax, VA 22030

29. NASA Goddard Space Flight Center, Greenbelt, MD 20771

30. IRFU/SEDI, CEA Saclay, 91191 Gif sur Yvette, France

31. Laboratoire de Physique Théorique et Astroparticules, Université Montpellier 2, CNRS/IN2P3, Montpellier, France

32. Department of Physics, Stockholm University, AlbaNova, SE-106 91 Stockholm, Sweden

33. University of Maryland, Baltimore County, Baltimore, MD 21250

34. Stellar Solutions Inc., 250 Cambridge Avenue, Suite 204, Palo Alto, CA 94306

35. Dipartimento di Fisica, Università di Udine and Istituto Nazionale di Fisica Nucleare, Sezione di Trieste, Gruppo Collegato di Udine, I-33100 Udine, Italy

36. ATK Space Products, Beltsville, MD 20705

37. CNRS/IN2P3, Centre d'Études Nucléaires Bordeaux Gradignan, UMR 5797, Gradignan, 33175, France

38. Université de Bordeaux, Centre d'Études Nucléaires Bordeaux Gradignan, UMR 5797, Gradignan, 33175, France

39. Department of Physical Science and Hiroshima Astrophysical Science Center, Hiroshima University, Higashi-Hiroshima 739-8526, Japan

40. University of Maryland, College Park, MD 20742

41. Orbital Network Engineering, 10670 North Tantau Avenue, Cupertino, CA 95014 
42. Department of Physics, Tokyo Institute of Technology, Meguro City, Tokyo 152-8551, Japan

43. Centre d'Étude Spatiale des Rayonnements, CNRS/UPS, BP 44346, F-30128 Toulouse Cedex 4, France

44. Center for Research and Exploration in Space Science and Technology (CRESST), NASA Goddard Space Flight Center, Greenbelt, MD 20771

45. Mätfakta i Kalmar AB, 30477 Kalmar, Sweden

46. Institute of Space and Astronautical Science, JAXA, 3-1-1 Yoshinodai, Sagamihara, Kanagawa 229-8510, Japan

47. Department of Physics and Astronomy, University of Denver, Denver, CO 80208

48. Dipartimento di Ingegneria dell'Informazione, Università di Padova, I-35131 Padova, Italy

49. Kavli Institute for Cosmological Physics, University of Chicago, Chicago, IL 60637

50. Current address: Pacific Northwest National Laboratory, Richland, WA 99352

51. Institut de Ciencies de l'Espai (IEEC-CSIC), Campus UAB, 08193 Barcelona, Spain

52. NYCB Real-Time Computing Inc., Lattingtown, NY 11560-1025

53. Department of Chemistry and Physics, Purdue University Calumet, Hammond, IN 46323-2094

54. Max-Planck-Institut für Kernphysik, D-69029 Heidelberg, Germany

55. Institució Catalana de Recerca i Estudis Avançats (ICREA), Barcelona, Spain

56. Consorzio Interuniversitario per la Fisica Spaziale (CIFS), I-10133 Torino, Italy

57. Current address: Yahoo! Inc., Sunnyvale, CA 94089

\begin{abstract}
The Large Area Telescope (LAT) on-board the Fermi Gamma-ray Space Telescope began its on-orbit operations on June 23, 2008. Calibrations, defined in a generic sense, correspond to synchronization of trigger signals, optimization of delays for latching data, determination of detector thresholds, gains and responses, evaluation of the perimeter of the South Atlantic Anomaly (SAA), measurements of live time, of absolute time, and internal and spacecraft boresight alignments. Here we describe on-orbit calibration results obtained using known astrophysical sources, galactic cosmic rays, and charge injection into the front-end electronics of each detector. Instrument response functions will be described in a separate publication. This paper demonstrates the stability of calibrations and describes minor changes observed since launch. These results have been used to calibrate the LAT datasets to be publicly released in August 2009 .
\end{abstract}


Keywords: GLAST, Fermi, LAT, gamma-ray, calibrations

PACS classification codes: $07.87 .+\mathrm{v} ;$ 95.55.Ka

\section{Introduction}

The Fermi Gamma-ray Space Telescope, hereafter Fermi, represents the next generation of satellite-based high-energy gamma-ray observatory. The Fermi satellite hosts two instruments: the Large Area Telescope (LAT) [1] and the Gamma-ray Burst Monitor (GBM) [2]. The former employs a pair-conversion technique to measure photons from $20 \mathrm{MeV}$ to energies greater than $300 \mathrm{GeV}$, while the latter uses $\mathrm{NaI}$ and BGO scintillation counters to record transient phenomena in the sky in the energy range from $8 \mathrm{keV}$ to $40 \mathrm{MeV}$. The LAT has no consumables, and a very stable response unlike its predecessor, the Energetic Gamma Ray Emission Telescope (EGRET) [3]. For the energy range above $10 \mathrm{GeV}$ the sensitivity of the LAT is at least one order of magnitude greater than that of EGRET, allowing the sky to be explored at these energies essentially for the first time [1].

The LAT consists of a tracker/converter (TKR) for direction measurements [4, 5, 6, 7], followed by a calorimeter (CAL) for energy measurements [8]. Sixteen TKR and CAL modules are combined to form sixteen towers, which are assembled in a $4 \times 4$ mechanical support structure. An anticoincidence detector (ACD), enclosed by a micrometeoroid shield, surrounds the TKRs and rejects charged cosmic-ray background [9, 10]. The LAT has about one million detector readout channels.

On-orbit calibrations relate to all aspects of LAT measurements and data analysis results, from absolute timing to energy and direction measurements for individual events, to fluxes and positions of gamma-ray sources.

The accurate timestamps of the LAT are obtained using the Global Positioning System (GPS) of the Fermi spacecraft, which provides timing and position information. Those are needed for phase folding pulsars and correlating gamma-ray observations with those at other wavelengths.

As the number of photons in an observation increases, the centroid of their spatial distribution becomes better measured, and eventually the error is dominated by uncertainties in the alignment of the LAT, both internal and with respect to the Fermi spacecraft.

Source localization at $\mathrm{GeV}$ energies enables the LAT to resolve bright, adjacent sources previously labeled as unidentified [3] and will help elucidate the origin of gamma-ray emissions from galactic cosmic rays accelerated in supernova remnants.

The energy calibrations at higher energies are of utmost importance for detection of dark matter particle signals. Some extensions to the Standard Model predict narrow spectral lines due to the annihilations of as-yet unknown massive particles. For detecting and characterizing these features, accurate energy determination is vital. Even though the LAT was designed to measure gamma-rays it can also study, though not separately, the cosmic-ray electron and positron spectra. Cosmic-ray electron and positron spectra and intensities may also contain signatures for new physics. In this case, energy calibrations and position determination using extrapolated tracks into the CAL play an important role. At lower energies, broad features in the photon energy spectra of active galactic nuclei, or supernovae remnants originating from pion decays and bremsstrahlung may help unravel outstanding questions 
concerning particle acceleration in these sources.

The purpose of this paper is to document the on-orbit calibration procedures used by the LAT; it begins with an overview of calibrations in Section 2. Details on trigger, ACD, CAL and TKR calibrations are described in Sections 3, 4, 5 and 6, respectively. The evaluation and updates to the perimeter of the South Atlantic Anomaly (SAA) are presented in Section 7 and measurements of live time are discussed in Section 8 . The results from absolute timing follow in Section 9, Finally, internal and spacecraft boresight alignments are explained in Section 10. We conclude with a table that summarizes the calibration results in Section 11. Assessment of the current LAT performance is described in a separate publication [11, while tests performed at particle accelerators are presented elsewhere [12, 13, 14]. The section describing each calibration in shown in the last column of Table 1.

\section{Overview of LAT calibrations}

For this paper, the word calibration represents synchronization of trigger signals, optimization of delays for latching data, determination of detector thresholds, gains and responses, evaluation of the perimeter of the South Atlantic Anomaly (SAA), measurements of live time and of absolute time and internal and spacecraft boresight alignments.

Table 1 summarizes all calibration types, classified by category. Configuration refers to operational settings that define the state of the hardware, which are fixed before data are acquired. Calibrations are related to quantities that can change after data are processed and analyzed. As shown in Table 1, for some types of calibrations, updates are not as frequent as the acquisition of the relevant data. Operationally, calibration data are acquired in two distinct modes (see Section 3.1):

1. Dedicated, meaning that the trigger, detector and software filter settings are incompatible with nominal science data taking.

2. Continuous, meaning the trigger and software can, with only a small penalty in live time, acquire specialized data that is used to calibrate, or, more generally, monitor the performance of the LAT during nominal science data-taking.

Within a run the LAT acquires data with fixed instrument configurations. For the most part, the dedicated calibration runs are concerned with characterizing the electronics' response to known stimuli while the continuous calibrations are aimed at calibrating the electronics with a known physics input. The stability of calibrations has been such that operations in dedicated-mode amount to approximately 2.5 hours every three months.

Sea-level cosmic ray muons were used to calibrate the low-energy scales and trigger thresholds, but muons do not deposit enough energy in the detector elements to calibrate the high-energy scales and high-energy trigger thresholds. Instead, we used charge injection into the front-end electronics to calibrate the high-energy scales. Because of rise-time slewing effects, the optimal synchronization of trigger signals and optimal delays for data latching are energy dependent. We used pre-launch tests to provide a best approximation of the optimal trigger timing, and verified and corrected the synchronization and delays with on-orbit data. All these calibrations are revisited with on-orbit data selected from galactic cosmic rays and, 


\begin{tabular}{|c|c|c|c|c|c|}
\hline Category & Title & Type & $\begin{array}{l}\text { Frequency to } \\
\text { acquire data }\end{array}$ & $\begin{array}{c}\text { Frequency of } \\
\text { updates } \\
\end{array}$ & Sec. \\
\hline Trigger & Time coincidence window & config & 1 year & 1 year & 3.2 \\
\hline Trigger & Fast trigger delays & config & 1 year & 1 year & 3.2 \\
\hline Trigger & Delays for latching data & config & 1 year & 1 year & 3.2 \\
\hline $\mathrm{ACD}$ & Pedestal & both & continuous & 3 months & 4.1 \\
\hline $\mathrm{ACD}$ & Coherent noise & calib & 3 months & 3 months & 4.1 \\
\hline $\mathrm{ACD}$ & MIP peak & calib & continuous & 3 months & 4.2 \\
\hline $\mathrm{ACD}$ & High range (CNO) & calib & continuous & 3 months & 4.2 \\
\hline $\mathrm{ACD}$ & Veto threshold & config & 3 months & 3 months & 4.3 \\
\hline $\mathrm{ACD}$ & High level discriminator & config & 3 months & 3 months & 4.3 \\
\hline CAL & Pedestal & both & continuous & 3 months & 5.1 \\
\hline CAL & Electronics linearity & calib & 3 months & 3 months & 5.2 \\
\hline CAL & Energy scales & calib & continuous & 6 months & 5.2 \\
\hline CAL & Light asymmetry & calib & continuous & 6 months & 5.3 \\
\hline $\mathrm{CAL}$ & Zero-suppression threshold & config & continuous & 3 months & 5.4 \\
\hline CAL & Low-energy threshold & config & continuous & 6 months & 5.4 \\
\hline $\mathrm{CAL}$ & High-energy threshold & config & continuous & 6 months & 5.4 \\
\hline $\mathrm{CAL}$ & Upper level discriminators & config & continuous & 1 year & 5.4 \\
\hline TKR & Noisy channels & config & continuous & 3 months & 6.1 \\
\hline TKR & Trigger threshold & config & 3 months & 3 months & 6.2 \\
\hline TKR & Data latching threshold & calib & 3 months & 3 months & 6.2 \\
\hline TKR & ToT conversion parameters & calib & 1 year & 1 year & 6.3 \\
\hline TKR & MIP scale & calib & continuous & 3 months & 6.4 \\
\hline SAA & SAA polygon & config & continuous & 1 year & 7 \\
\hline Timing & LAT timestamps & calib & continuous & continuous & 9 \\
\hline Alignment & Intra tower & calib & continuous & 1 year & 10.1 \\
\hline Alignment & Inter tower & calib & continuous & 1 year & 10.2 \\
\hline Alignment & LAT boresight & calib & continuous & 1 year & 10.3 \\
\hline
\end{tabular}

Table 1: List of LAT calibrations (calib) and configurations (config) that can impact LAT scientific results. Note that pedestals are used both as a configuration on-board and as a calibration on the ground. Detailed descriptions are given in the sections listed in the last column. Frequency of updates correspond to current best estimates for the period beyond the first year of operations. 
as it will be shown later, there are only minor deviations when comparing results prior and after launch.

\section{Overview of trigger and readout}

The trigger and data readout system controls the composition and flow of data from the source in the detector elements to the Solid State Recorder (SSR) of the Fermi spacecraft. There are two distinct stages: the latching and movement of data from the front-end detector elements to a LAT Event Processing Unit (EPU), and the processing of the data on the EPU and its subsequent transfer to the SSR. The first stage is controlled by hardware, namely the trigger, and the second stage by software. A full description of the LAT multilevel trigger and data readout can be found elsewhere [1, 15].

\subsection{Acquisition modes: dedicated or continuous}

During dedicated calibration runs the trigger system and detector electronics are configured to acquire data useful for calibrating the thresholds and responses of detectors and synchronizing the arrival times of signals from various parts of the detectors. We call these runs dedicated because the trigger and detector configurations necessary to acquire these specialized data are incompatible with acquiring our primary science data (i.e. high-energy gamma rays from celestial sources). During continuous calibration acquisitions, the trigger system and detector electronics are configured to detect and latch not only those events thought to be gamma rays, but also those useful for calibration.

As used on orbit, the LAT trigger system takes inputs from the ACD, TKR, and CAL front-end electronics and from a programmable, internal periodic trigger. Under control of LAT flight software, the periodic trigger can be used with a programmable charge-injection system to calibrate the detector electronics, or it can be used simply to read out the detector front-ends at a specified cadence.

The programmed control under the charge injection system is used only in dedicated calibration runs. There the trigger is configured to collect a specified number of events at a particular rate, regardless of input from the detectors. In most cases, the trigger is configured to instruct the detector electronics to inject a known, programmable amount of charge at a specified time relative to each trigger. In other cases, no charge is injected. For each sequence of these events, flight software sets configuration registers throughout the instrument that control, for example, the amount of charge injected. By collecting a number of events with the same injected charge, and then varying the amount of injected charge, the electronic response can be accurately calibrated.

Other dedicated calibration runs and all continuous calibration runs have the instrument triggered by signals from the detectors, not a programmed sequence, and charge injection is not used. For example, dedicated calibration runs in this mode are those used to synchronize trigger signals from the detectors. Data are collected using a sequence of configurations that sweep values through the various registers that control the delay times of the trigger signals. The data captured are then analyzed on the ground to identify the delay settings that best synchronize the arrival times of the trigger signals from each detector system. 
During continuous calibration runs, the trigger is configured to detect and latch both events thought to be gamma rays and other events useful for calibration. Based on the signals recieved from the detectors, the trigger specifies the type of ACD and CAL readout. There are four types (see Table 2) formed by enabling or disabling zero-suppression, in which values are committed to the data stream only if they exceed a programmable threshold, and selecting CAL single-range or four-range mode, in which the electronics takes respectively only the "best" range or all four values (see Section 5.4 for details). The ACD does not support a readout mode to select both readout ranges. It does, however, respect the zerosuppress mode.

\begin{tabular}{|c|c|}
\hline \hline Purpose & Readout Mode \\
\hline \hline Select photons & $\begin{array}{c}\text { Zero-suppressed } \\
\text { and best range }\end{array}$ \\
\hline Cross-calibration of the energy ranges (CAL) & $\begin{array}{c}\text { Zero-suppressed } \\
\text { and four-range }\end{array}$ \\
\hline Calibration of the MIP peak (CAL,ACD) & $\begin{array}{c}\text { Zero-suppressed } \\
\text { and best range }\end{array}$ \\
\hline Monitor pedestals (CAL,ACD) and noise occupancy (TKR) & $\begin{array}{c}\text { Non-zero-suppressed } \\
\text { and four-range }\end{array}$ \\
\hline \hline
\end{tabular}

Table 2: Readout modes used for continuous calibrations (nominal science operations).

After an event passes the hardware trigger it is inspected by on-board software filters, each configured to identify events likely to be useful for one or more scientific or calibration purposes. If any filter accepts an event, it is included in the LAT data stream and forwarded to the SSR for transmission to the ground. In addition, the filters can be configured to allow a fraction of events that would otherwise have been rejected to be included in the data stream. Such events are used primarily to study filter performance. The input trigger rate of about $2.2 \mathrm{kHz}$, averaged over many orbits, is reduced to about $450 \mathrm{~Hz}$ by the software filters. Table 3 describes filter types, purpose and average output rate.

\subsection{Synchronization of trigger signals and delays for latching data}

Fast trigger signals ( few hundred ns) from the detectors must be synchronized with respect to each other for the trigger to operate efficiently. There are five such signals: veto and high level discriminator in the ACD (see Section 4), low and high-energy in the CAL (see Section 5 ) and TKR (see Sec 6). The earliest arriving trigger signal initiates a readout. The arrival times of the other trigger signals relative to this signal are captured in the event data with 50 ns precision (i.e. the period of the LAT system clock), allowing a direct comparison of trigger signals on an event-by-event basis. Once the relative timing is determined, the settings of various delay registers (i.e. the instrument configuration) are modified to synchronize these trigger signals in subsequent data acquisitions. There are two types of delay associated with the trigger: the fast trigger signal delay and the delay for latching the data. To synchronize 


\begin{tabular}{|c|c|c|}
\hline \hline Filter Type & Purpose & Average Rate (Hz) \\
\hline \hline Gamma & $\begin{array}{c}\text { select gamma-ray candidates } \\
\text { and events }>20 \mathrm{GeV}\end{array}$ & 410 \\
\hline Heavy ion & calibration of high-energy scales, & 2.5 \\
\hline MIP & select non-interacting protons & $\begin{array}{c}0 \text { (nominal) } \\
\text { (dedicated-mode) }\end{array}$ \\
\hline Diagnostic & filter performance, background & 22 \\
\hline \hline
\end{tabular}

Table 3: On-board filters used to select events for calibration acquisitions. Rates depend on geomagnetic and other orbital variations. Here we list average rates.

\begin{tabular}{|c||c|c|c|}
\hline Measurement & ACD Delay & TKR Delay & CAL Delay \\
\hline \hline Ground & 800 ns $(16$ ticks $)$ & 250 ns $(5$ ticks $)$ & 0 \\
\hline Orbit & 750 ns $(15$ ticks $)$ & 200 ns $(4$ ticks $)$ & 0 \\
\hline
\end{tabular}

Table 4: Trigger signal delays for measurements on the ground and on orbit.

the detectors we use the time of arrival of trigger signals, referred to as condition arrival times. This is event information containing the number of 50 ns clock ticks that have passed between the opening of the coincidence time window and the arrival of a given trigger signal. Since the trigger signals for TKR and CAL have significant time walk depending on the ratio of signal size to threshold value, it is important to choose an appropriate dataset to optimize the timing. We select MIP data for the relative timing of ACD and TKR, since the main purpose of the ACD is to reject charged particle background. The relative timing between TKR and CAL is optimized using photon candidates selected by the gamma filter. The CAL low and high-energy signals are controlled by a single delay and cannot be tuned independently.

Figure 1 shows the arrival times for CAL with respect to the TKR. Negative values indicate that the TKR trigger arrived earlier than the CAL trigger signal. Distributions are fully contained inside the time coincidence window $\oplus^{\circ} 700 \mathrm{~ns}$ (14 ticks). The arrival times are affected by the ratio of the crystal energy over the threshold, thus influencing the shape of the trigger signal curves. The spectrum is slightly altered from its original form due to a trigger event selection. There is no difference in arrival times between CAL high- and CAL low-energy triggers at energies very far above the two thresholds.

On the ground, only muons were available for timing calibrations so a small change in parameters was observed on orbit. The results from the synchronization of the trigger signals are shown in Table 4 .

The optimal delay is obtained after analyzing data acquired with fixed delay values. For the CAL we fit the MIP peak for each dataset with a fixed delay. Figure 2 a shows an example for a delay setting of 750 ns (25 ticks). Figure $2 \mathrm{~b}$ shows CAL MIP peak positions for six

\footnotetext{
${ }^{\dagger}$ Prior to launch the time coincidence window was set to $600 \mathrm{~ns}$ (12 ticks).
} 


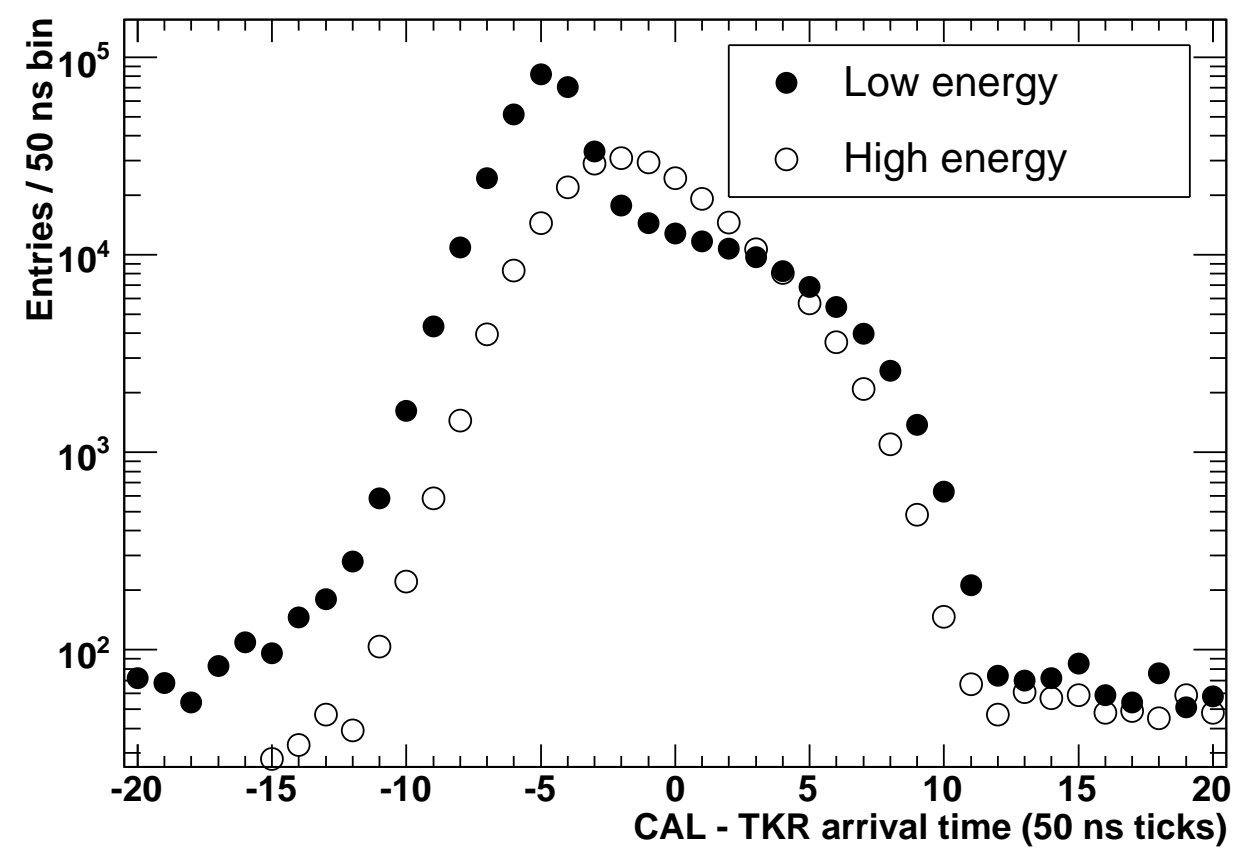

Figure 1: On-orbit arrival times of the CAL low-energy (FLE) and high-energy (FHE) trigger signals with respect to the TKR trigger. This special dataset was acquired with the time coincidence window of $1550 \mathrm{~ns}$ (31 ticks). Most of the entries fall within the window used for nominal science operations (700 ns or \pm 13 ticks). 

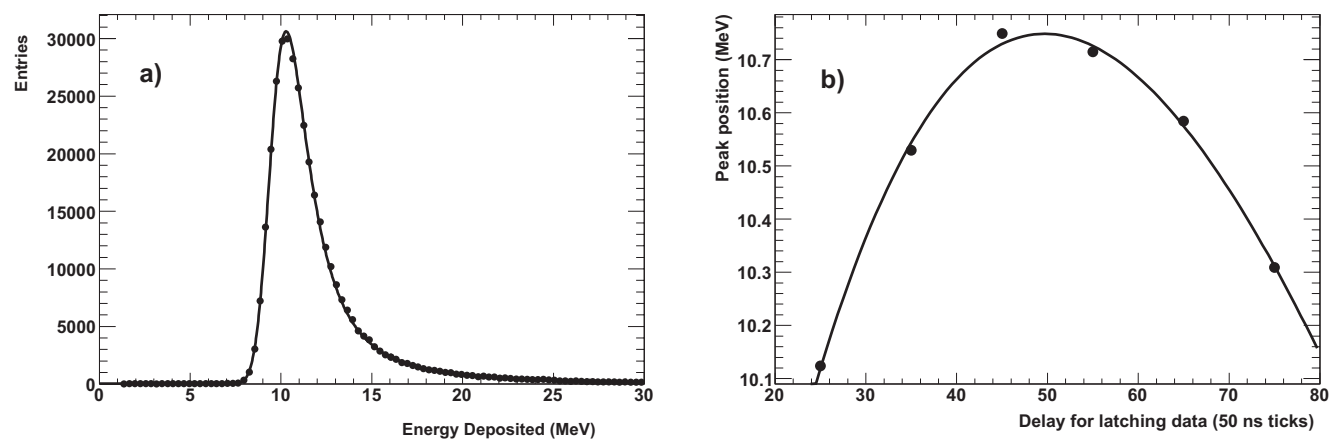

Figure 2: a) Energy deposited in a single CAL crystal (averaged over all towers) for a fixed value of the delay for latching data. The fit (curve) is used to determine the MIP peak position in the CAL, b) MIP peak positions for different CAL delay values (all towers). The curve is a parabola fit to the data.

delay values for latching data. The optimal setting is the peak position obtained from the fit to the data. There is a data point slightly off the curve due to changes in geomagnetic conditions along the orbit. Since data were not recorded at the same location in orbit, the MIP selection cuts are designed to keep variations to $<0.1 \%$ of the MIP peak value. A similar procedure is applied to the ACD. For the TKR, the quantity of interest is not the MIP peak position but instead the detector efficiency, which is defined as the number of layer hits between the first and the last hit of the track divided by the expected number of layers

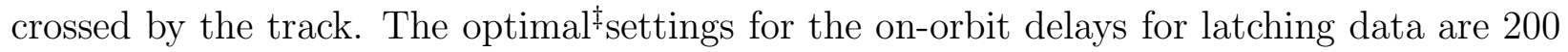
ns (4 ticks), 0 and 2450 ns (49 ticks) for the ACD, CAL and TKR delays, respectively.

\section{ACD calibrations}

The ACD is the LAT first-level defense against the charged particle cosmic-ray background that outnumbers the gamma-ray signals by 3-5 orders of magnitude. It consists of 97 separate plastic scintillating detectors - 89 scintillator tiles and 8 scintillator ribbons, each viewed by two photomultiplier tubes (PMTs) for redundancy. The overall ACD detection efficiency is $>0.9997$, which is provided by ensuring high uniformity of the detectors' response and a large number of photoelectrons. The segmentation is needed in order to minimize pulse height variations over the ACD area and to minimize unwanted self-veto due to backsplash of soft photons from the developing electromagnetic shower in the CAL. Such self-veto caused a significant reduction in effective area in EGRET at high energies [3, 16]. Detailed information about the ACD can be obtained elsewhere [9, 10].

ACD calibrations include the determination of the mean values of pedestals, of the signal pulse heights produced by single MIP particles in each ACD scintillator, and the veto threshold settings; and the high-energy and coherent noise calibrations. All those parameters are determined for each PMT, so that each ACD tile or ribbon has two calibrated values. Every ACD channel has two ranges, low and high, in order to expand the dynamic range

\footnotetext{
${ }^{\ddagger}$ The efficiency for latching TKR data is unchanged up to $\geq 750 \mathrm{~ns}$ (15 ticks).
} 

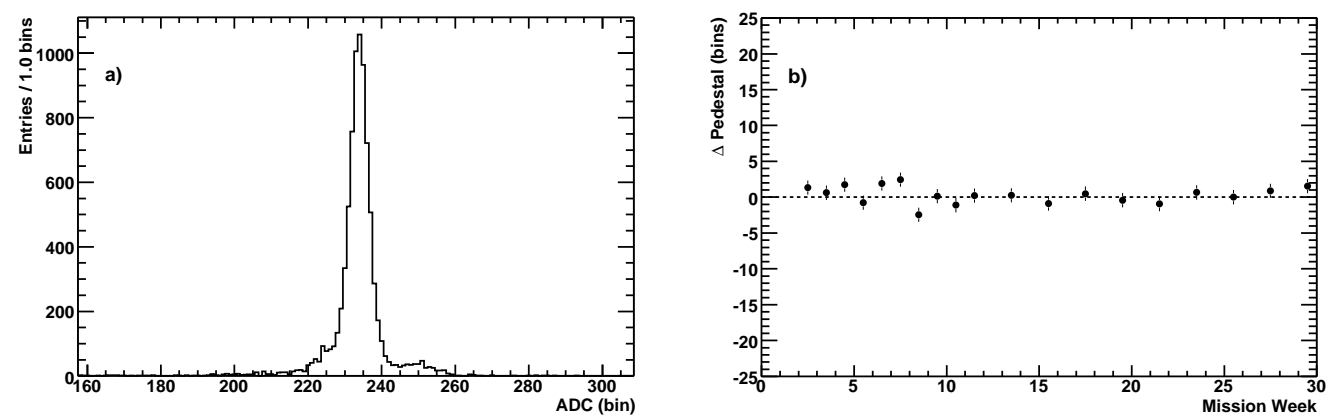

Figure 3: a) Pedestal distribution from periodic triggers for a single PMT, b) Long term pedestal trending for a single PMT.

of processed signals. The low range covers the signals below 4-8 MIPs (depending on the channel), and the high range extends well above 1000 MIPs. The switching between ranges occurs automatically depending on the amplitude of the signal.

In the future there may be changes to the MIP peak positions due to degradation of PMT photocathodes, scintillators or in an optical path between them. A possible way to mitigate these effects is to raise the high voltage for the PMTs. Since each high voltage is common to groups of 16 or 17 PMTs, this requires re-calibration of all channels belonging to that particular PMT group.

\subsection{Pedestals and coherent noise}

Pedestals are offset voltages present at the Analog-to-Digital Converter (ADC) inputs in the low and high range readouts. We extract the ACD pedestals for the low range readout from the $2 \mathrm{~Hz}$ periodic triggers, which currently provide approximately 10,000 random samples per orbit. Since a small fraction of these events contains particle signals or electronics noise or tails from previous signals in the ACD, we extract the pedestal values by performing a Gaussian fit to the central $80 \%$ of the pulse height distribution (truncated mean). Figure. 33 shows a typical pedestal distribution for a single PMT, where the width of the Gaussian (truncated) is about $2-4$ pulse height bins ( $\sim 0.01 \mathrm{MIPs})$. The narrow core of the distribution shows the intrinsic electronic noise; tails are residual signals from particles near in time to the periodic trigger. The small peak at about 250 is dominated by coherent noise contributions. Extracting the pedestal for the high range readout requires a special data-taking configuration, which forces a series of randomly triggered events to be read out in the high range. Since this configuration is incompatible with regular data taking and the pedestals are reasonably stable, these data are only acquired during the quarterly calibration periods where the LAT is in dedicated-mode. The data analysis is similar to that of the low range pedestals. Figure $3 \mathrm{~b}$ shows the long term pedestal trending data for a single PMT. All values are plotted relative to the calibration being used, at the time of writing, in the offline reconstruction software (Mission week 25). To reduce data volume, we use the low range pedestal values on-board. We reject all signals less than 25 counts above pedestal, which corresponds to approximately 8 times the electronics noise, or 0.05 times the MIP signal in 


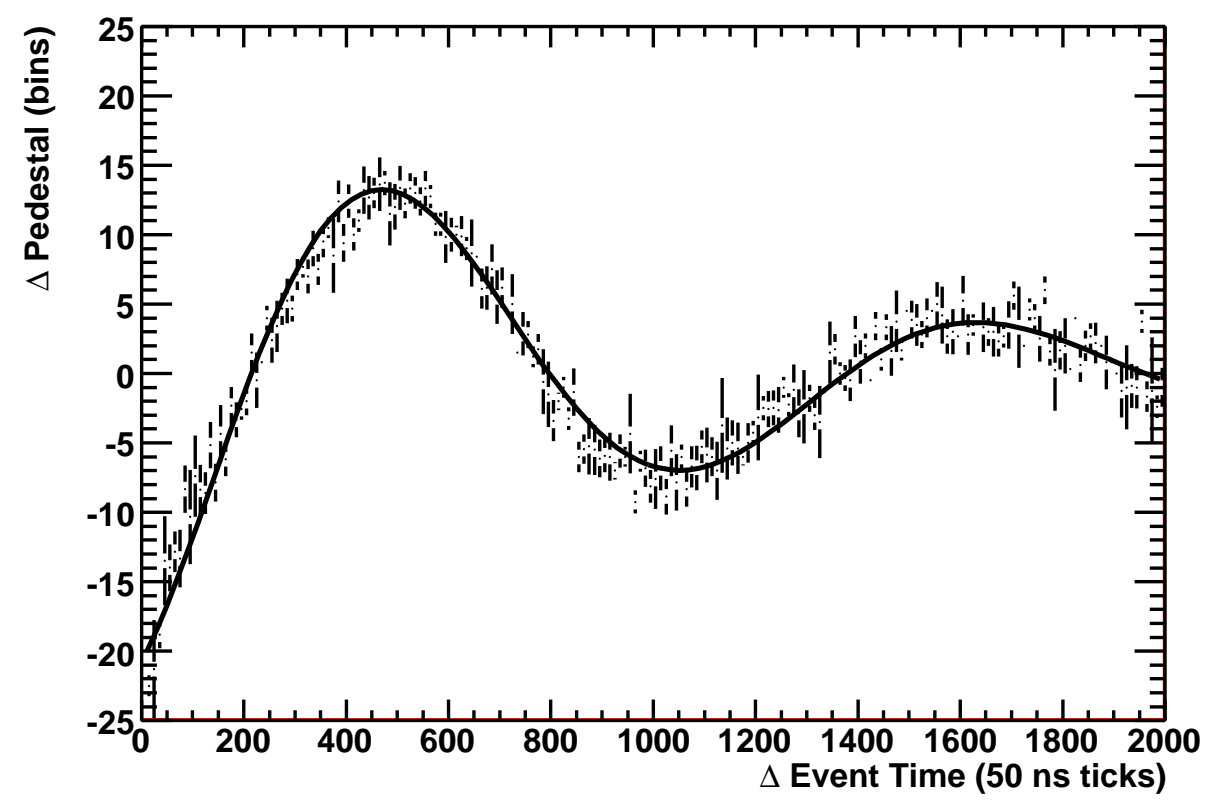

Figure 4: Readout related pedestal ringing in a single PMT. The truncated mean and RMS ADC values obtained using periodic triggers versus the time difference between consecutive readout cycles in 50 ns clock ticks. The curve corresponds to a fit of a sinusoidal oscillation inside a decaying exponential envelope.

a typical tile. We also use pedestal values in the flight software data compression algorithm (for both $\mathrm{ACD}$ and $\mathrm{CAL}$ ). In this case we reduce the data size by referencing signals against pedestal values rather than against zero.

During ground testing of the ACD we discovered that the readout process causes the electronics pedestals to ring. These oscillations occur when elements of its internal circuitry resonate at their characteristic frequency. This reproducible effect can be quantified. Figure 4 shows the difference ( $\Delta$ Pedestal) between the coherent noise and the regular pedestal values for a single PMT. This truncated mean (see Section 4.1) is displayed versus the time difference between consecutive readout cycles in 50 ns clock ticks. Data were obtained using periodic triggers and the error bars correspond to the RMS ADC values. The curve represents a fit to a sinusoidal oscillation inside a decaying exponential envelope, which falls to the electronics noise after about $200 \mu \mathrm{s}$.

As this effect is present in every channel of the ACD, events read out when the pedestal peaks at about $50 \mu \mathrm{s}$ (1000 ticks) after the previous readout can lead to small signals in many PMTs. This correction is applied to the offline data to avoid overestimating the total energy in the ACD and compromising background rejection and photon selection. For events taken at the peak of the coherent oscillation $(\sim 500$ ticks $)$, this calibration reduces the coherent noise contribution from 0.05 MIPs per PMT to less that 0.005 MIPs per PMT. The effect is so small that we do not need to apply corrections to the on-board processing. Temperature dependences in ACD pedestals are negligible. 

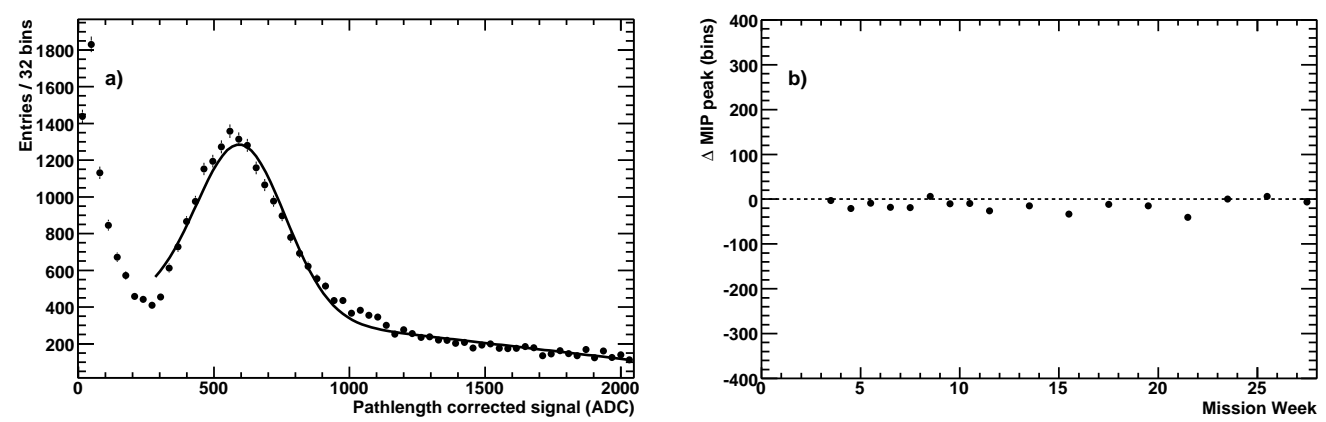

Figure 5: a) Distribution of pedestal subtracted and pathlength corrected signals for a single PMT in the ACD; the MIP peak is clearly visible and the curve is a fit to the data, b) Long term trending of the MIP peak versus mission week.

\subsection{MIP peak and high range calibrations}

The MIP peak values are determined using reconstructed tracks pointing at an ACD tile or ribbon that recorded a signal. The peak value of the pathlength corrected pulse-height distribution for each PMT is the MIP peak for that channel. This calibration is a heuristic attempt to quantify the average MIP signal seen in the ACD and not a precise determination of all details of the energy deposition in the ACD sensors. Figure 5 a shows a distribution of pedestal subtracted and pathlength corrected signals for a single PMT in the ACD. The MIP peak at about 600 is clearly seen and the peak below 100 corresponds to soft Xray background. Figure $5 \mathrm{~b}$ displays the long term trending of the MIP peak for a single PMT, where pedestals from mission week 3 are used as a reference. The stability of the measurement is about $10 \%$ of the MIP peak for a period of 20 weeks. During the offline reconstruction we use the MIP peak calibration values to express raw signal pulse height measurements in MIP equivalent values. These are converted into energy using the energy deposition in the scintillator $(\sim 2 \mathrm{MeV} / \mathrm{cm})$. MIP peaks in the ACD tiles occur between 400 and 1000 pulse height bins above pedestal, and are determined with an accuracy better than $5 \%$.

The high and low range readout are calibrated in an analogous way. The main difference is that for the high-range readout we use tracks identified as carbon nuclei by the CAL, since the proton MIP-like signals are too small. The algorithm requires the deposited energy in the CAL, which is pathlength corrected, to be consistent with that of carbon and the first few layers to have similar energy to avoid carbon interacting events. Also, in converting from raw pulse heights to MIP equivalent values we allow for non-linearities caused by signal saturation in the electronics and scintillators. Figure 6 shows the deposited energy for the full dynamic range of a single PMT. The solid line shows events read out in the low range and the dotted line shows events read out in the high range. Figure 7 shows a distribution of pedestal subtracted and pathlength corrected signals for carbon in a single PMT. 


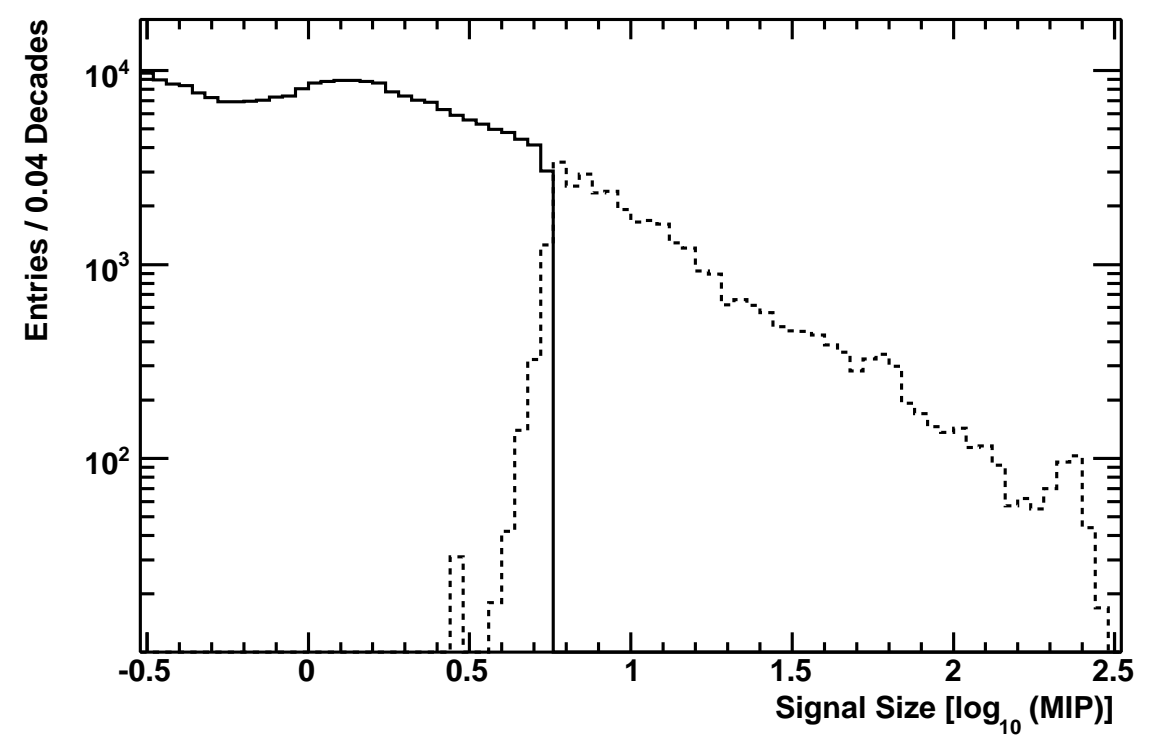

Figure 6: Deposited energy for the full dynamic range of a single PMT. The solid line shows events read out in the low range, the dotted line shows events read out in the high range. The MIP peak occurs $\sim 1.3$ MIPs because pathlength corrections are not applied.

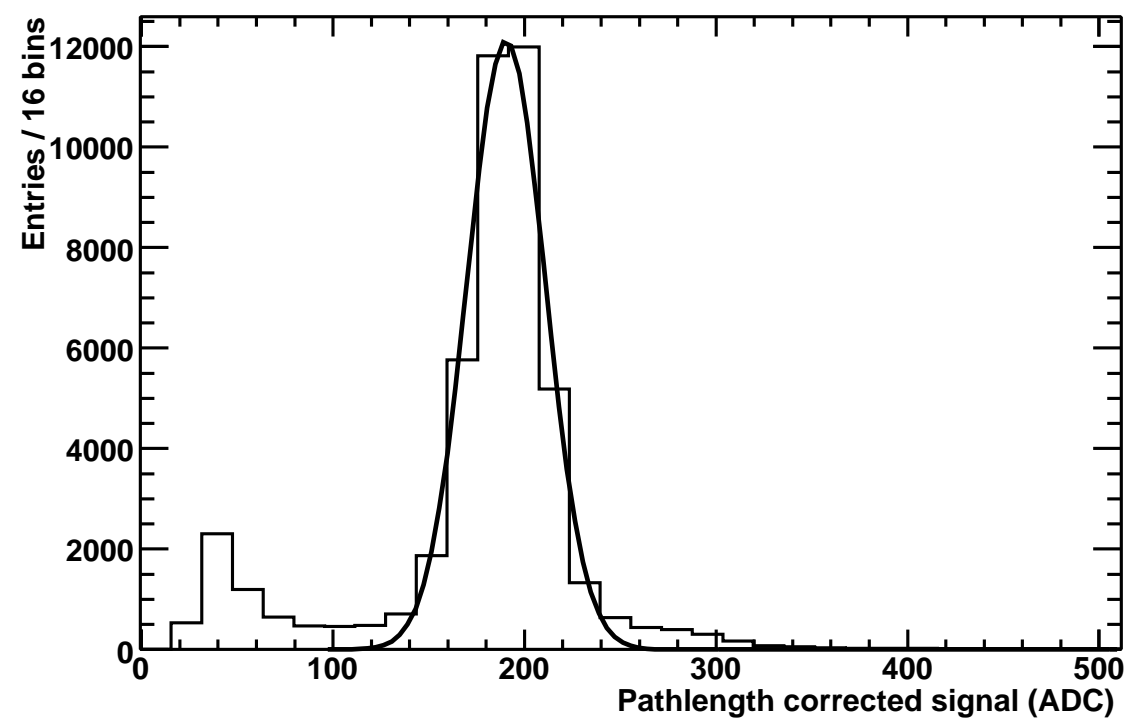

Figure 7: Distribution of pedestal subtracted and pathlength corrected carbon signals for a single PMT in the ACD in the high range. The carbon peak is clearly visible at $\sim 200$ counts and the curve shows the fit to the data. 


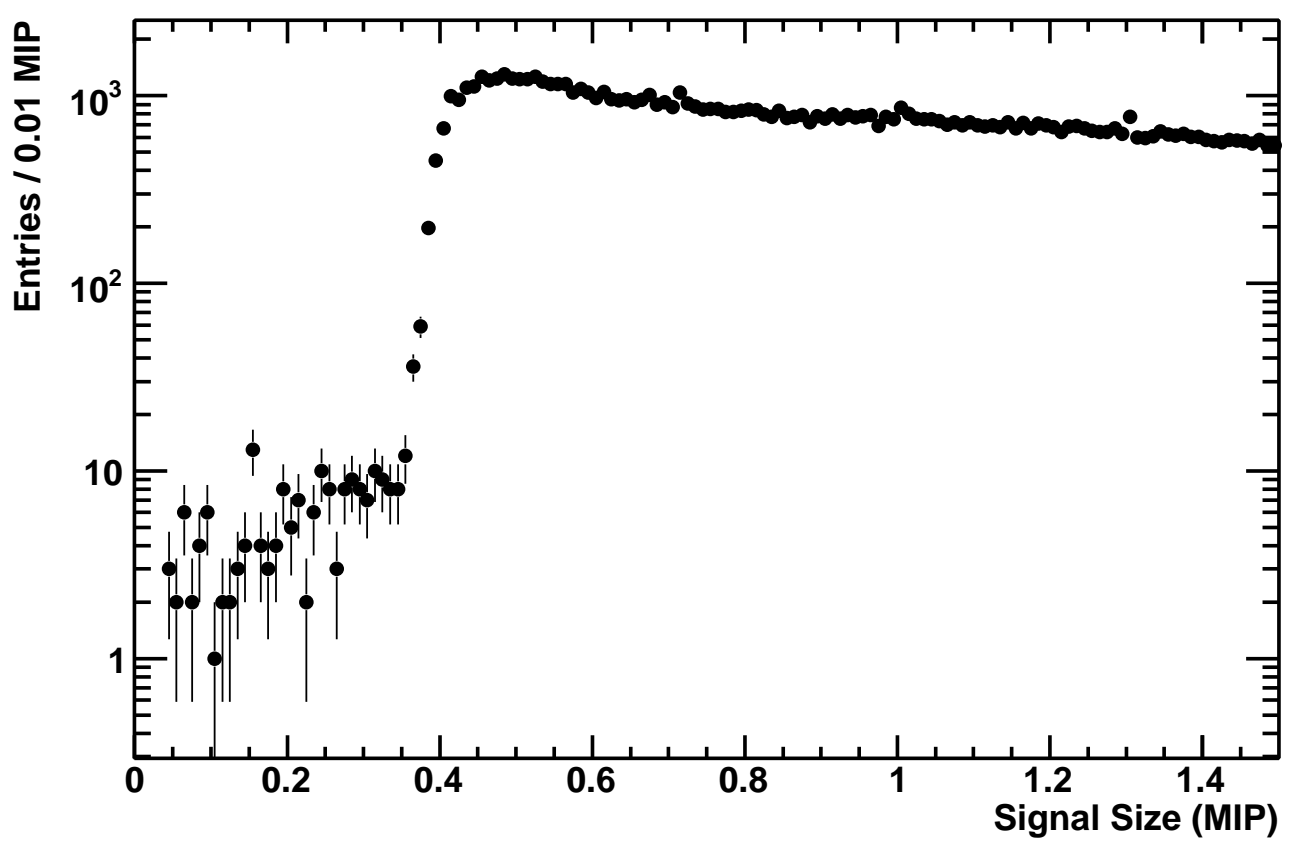

Figure 8: Veto turn-on curve for a single PMT. The small number of events $(0.1 \%)<0.4$ MIPs correspond to overshoots in the ADC value from a previous event.

\subsection{Veto threshold and high-level discriminator}

The on-board thresholds are controlled by DAC settings in the front-end electronics. These are calibrated by scanning three settings and measuring the resulting veto threshold in pulse height bins. We combine that information with the MIP peak to pulse height scale, or carbon peak for the high range, and set the veto and high-level discriminator thresholds as a fraction of the MIP and carbon signals, respectively. The veto thresholds for each PMT are set separately to an accuracy of about 0.01 MIPs $(\sim 20 \mathrm{keV})$ relative to the calibrated MIP peak. As shown in Fig 8 , the veto turns on between 0.4-0.5 MIPs and the 50\% efficiency point is close to 0.45 MIP. For events below $<0.4$ MIPs the ADC values can be artificially small, because the readout electronics are relatively slow when compared to the veto electronics. Events arriving when the electronics are overshooting the return to baseline tend to fire the veto discriminator, even though they are not expected to have any ADC counts. The turn-on for the high level discriminator occurs between 24-26 MIPs and the RMS width of the carbon peak is $20 \%$ of the mean value. We have not yet monitored the stability of the carbon peak since it needs large statistical samples. At least four months of data are required to obtain a carbon peak value with reasonable statistics.

\section{CAL calibrations}

The CAL is designed to measure the energy of incident photons and charged particles, and to determine the direction and energy of photons and charged particles for which the TKR 
did not provide direction information, either because their trajectories did not cross the TKR or because they did not pair-produce in the TKR. Its imaging properties are also a key ingredient in seeding the track reconstruction process in TKR data analysis and in the rejection of charged-particle background [1].

The CAL consists of 16 identical modules. Each module is composed of $96 \mathrm{CsI}(\mathrm{Tl})$ scintillation crystals arranged in a hodoscopic configuration with eight layers each containing 12 crystals. Each layer is rotated $90^{\circ}$ with respect to its neighbors, forming an $x-y$ array. Crystals are read out by two dual-PIN-photodiode assemblies, one at each end, that measure the scintillation light produced in the crystal. Each photodiode assembly contains a largearea photodiode to measure small energy depositions and a small-area photodiode to measure large energy depositions. The active areas of the large and small diodes have a ratio of 6:1 with a spectral response well matched to the scintillation spectrum of $\mathrm{CsI}(\mathrm{Tl})$. Each of the 3072 photodiode assemblies is read out by an amplifier-discriminator ASIC, the GLAST Calorimeter Front-End Electronics (GCFE). To cover a large dynamic range of $5 \times 10^{5}$ in each GCFE with commercially available 12-bit ADCs for digitization, the low and high energy photodiodes each have their own independent signal chains, the low-energy and the high-energy, and each chain operates with two track and hold gains (low and high). This arrangement results in four overlapping energy ranges from $2 \mathrm{MeV}$ to $70 \mathrm{GeV}$ overall, as shown in Table 5. Range overlap allows cross-calibration of the electronics. Table 5 also shows the approximate factors used to convert ADC readout to energy units $(\mathrm{MeV})$.

A detailed description of the CAL is found elsewhere [8].

\begin{tabular}{|c|c|c|l|c|}
\hline Name & Energy & Gain & Energy range & MeV/ADC \\
\hline LEX8 & low & high & $2 \mathrm{MeV}$ to $100 \mathrm{MeV}$ & 0.033 \\
\hline LEX1 & low & low & $2 \mathrm{MeV}$ to $1 \mathrm{GeV}$ & 0.30 \\
\hline HEX8 & high & high & $30 \mathrm{MeV}$ to $7 \mathrm{GeV}$ & 2.3 \\
\hline HEX1 & high & low & $30 \mathrm{MeV}$ to $70 \mathrm{GeV}$ & 20 \\
\hline \hline
\end{tabular}

Table 5: Four overlapping readout ranges for the CAL and their conversion factors.

Here we describe the on-orbit measurements of the following: CAL pedestals; crystal energy scale, derived from the electronics linearity and crystal light output; crystal light asymmetry, which calibrates position measurements along the crystal; and threshold settings. These calibrations allow determination of the location and amount of energy deposited in each crystal. Processes for estimation of incident photon energy and the resulting overall energy resolution are discussed elsewhere [11, 14].

\section{$5.1 \quad$ Pedestals}

As discussed in Section 4.1, pedestals are offset voltages for each of the four CAL energy ranges that set the "zero point" for the energy scale. We measure pedestals on orbit from the periodic triggers issued at $2 \mathrm{~Hz}$ by the LAT trigger system during all nominal science data acquisitions. Chance coincidence energy deposits result in a small tail to the pedestal distribution, but this is suppressed both by the pedestal distribution fitting techniques used and by comparison of the various energy ranges. 

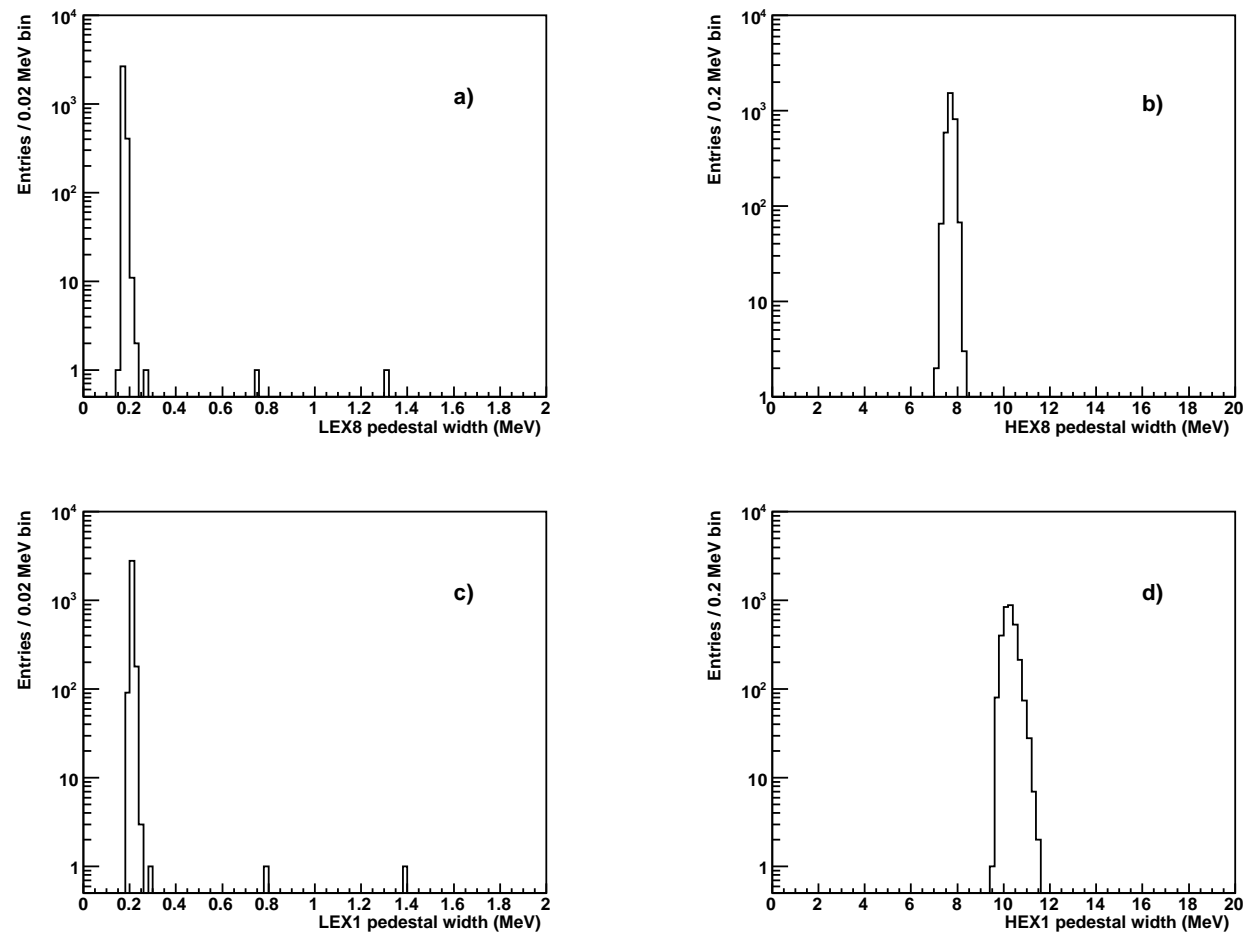

Figure 9: On-orbit pedestal widths (for all channels) for: a) LEX8, b) HEX8, c) LEX1 and d) HEX1 ranges.

Figure 9 shows that typical pedestal widths (RMS) are $0.2 \mathrm{MeV}$ for the LEX1 and LEX8 ranges and 7-10 MeV for the HEX1 and HEX8 ranges. The pedestal values are regularly monitored on orbit and have been extremely stable since an initial settling period.

Pedestal measurements made during thermal-vacuum tests in January 2008 indicated a channel-dependent linear dependence of pedestal position with temperature, where the drift magnitude varies from -3 to +3 ADC units per degree for the LEX8 and HEX8 ranges and $\sim 10$ times smaller for LEX1 and HEX1, reflecting their smaller gains.

When Fermi is in Pointed observing mode, the exposure of the LAT thermal control system to the warm Earth changes relative to the Sky Survey observing mode, and the LAT detector temperatures change modestly. In the CAL, the temperature changes 1-2 degrees within a few hours, leading to a temperature drift of the pedestals of a few ADC units at most. Since Log Accept (LAC) thresholds, which determine whether a given crystal readout is included in the data stream, are set in absolute ADC unit values (i.e. not relative to the pedestal), changes in the pedestal values change the corresponding threshold energies. A large enough pedestal change could thus influence data volume. However, only very large temperature changes $(\sim 20$ degrees Celsius), which are not anticipated during the mission, could lead to significant increases in data volume. 

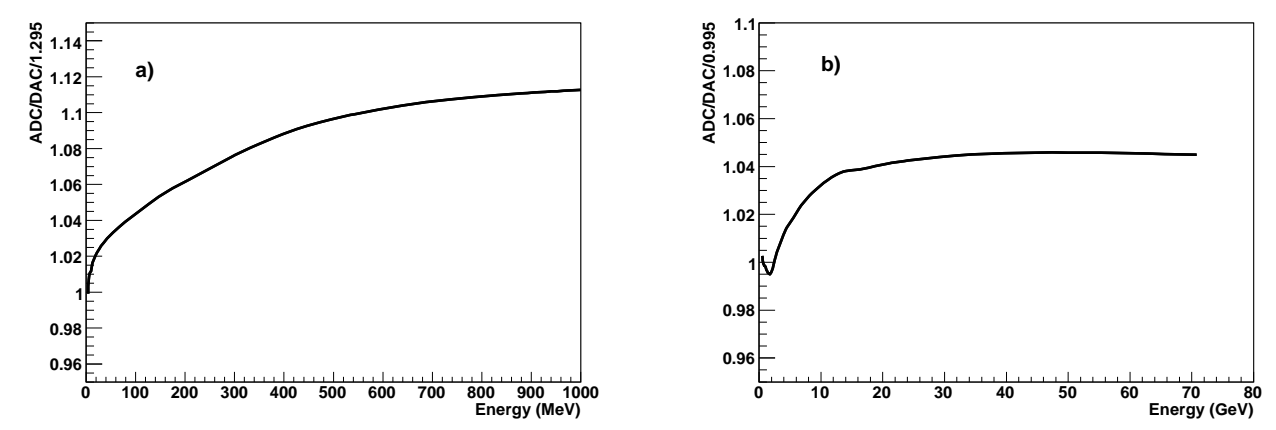

Figure 10: Characterization of electronics non-linearities. Normalized ADC/DAC versus energy for: a) LEX1 and b) HEX1 ranges.

\subsection{Individual crystal energy scales}

Calibration of the individual crystal energy scales involves the determination of the parameters of a transfer function between the energy deposited in the crystal and the signal output in ADC units. The transfer function consists of the response of the front-end electronics and the light emission and collection properties of the scintillation crystals. We use charge injection calibrations to describe the electronics and ionization energy depositions from cosmic-ray muons or protons and various nuclei to calibrate crystal response. Ionization energy losses over a known path length are very predictable and hence make a good calibration tool.

Charge injection calibrations are used to characterize the non-linear behavior of the electronics chain. A pulsed signal of known amplitude (controlled by a charge injection calibration DAC) is sent to the preamplifier input of each CAL front-end electronics channel. The nonlinearity of this DAC is specified by the manufacturer to be $<0.1 \%$ and hence negligible when compared to the nonlinearity of the front-end electronics.

For each fixed DAC setting we inject 100 pulses onto each of the electronics channels and average the resulting ADC output values. A spline function is used to fit the resulting DAC versus ADC curve. The functions (one for each channel), describe both electronics gain and non-linearity. Using these functions, signals from each channel can be converted to a linear scale. Figure 10 shows the normalized ADC/DAC versus energy, where deviations from one indicate non-linear behavior for measurements made at fixed energy values. The largest deviations $(\sim 12 \%)$ are seen in the LEX1 range displayed in Figure 10a. Figure 10b illustrates the case for the HEX1 range with $4 \%$ non-linearities. The feature around $2 \mathrm{GeV}$ in the HEX1 curve corresponds to cross-talk between LEX1, which saturates around $1 \mathrm{GeV}$, and HEX1. For the other ranges (LEX8 and HEX8) deviations are $<1 \%$. After applying the measured nonlinearity calibration, residual nonlinearity is $\leq 1 \%$ of the measured energy, resulting in a negligible systematic effect in spectrum determination.

The crystal response calibration, i.e. the function that relates deposited energy to the linearized signal described above, has been performed using different ionizing particles on the ground and in orbit. In both cases, ion incident energies are, for the most part, in the slow relativistic rise region of the Bethe-Bloch curve, so the predicted energy loss per unit path length $\left(\frac{d E}{d x}\right)$ is only weakly dependent on incident energy. Using simulated incident spectra for ground and orbit environments, we determine an expected $\frac{d E}{d x}$ for each incident 
particle species. We then collect spectra of the these species, correcting each event for path length, and compare the peak position in pulse height units to the predicted position in energy to yield a calibration. Variations in incident spectrum with orbital position result in slight broadening of the energy deposit peaks, but, given integration over multiple orbits, the peak most-probable-value is well-determined and usable for calibration.

On the ground, the low-energy scales were calibrated using sea-level cosmic ray muons while high energy ranges were calibrated using muons with the HEX1 and HEX8 channels set to a special "muon mode" gain setting that increased the gain by a factor of $\sim 10$.

On-orbit, we used a technique we refer to as "proton inter-range calibration". The lowenergy scales are calibrated using protons. Higher energies are calibrated by using energy deposits that meet two criteria: first they must be in the overlap range between LEX1 and HEX8 and second they must result in a "heavy ion" trigger, the only common trigger that produces the required 4 energy range readout rather than the normal single range readout. These events are a combination of galactic cosmic ray (GCR) primary carbon nuclei, interacting protons and other interacting or ionizing GCRs. From events that meet these criteria, we can construct a cross calibration of the low and high energy ranges. In both ground and on-orbit cases, the ionization calibration, together with the charge injection results yield a usable energy scale that converts ADC units to deposited energy. A week of nominal science operations data is sufficient to calibrate the energy scales using relativistic protons and heavy ion trigger events in the overlap energy range.

Protons that are accepted by either the MIP filter (when active) or the diagnostic filter (see Table 3) are required to pass a number of cuts. In order to reject all events that are not contained in a single CAL module and to eliminate "corner clipping" events, for which path length determination is not sufficiently accurate, we require extrapolated TKR tracks to cross the top and the bottom surfaces of a single CAL crystal, and be at least $5 \mathrm{~mm}$ away from the crystal edges. In addition, we require single TKR track events with $>20$ TKR hits and a chi-square for the track $<3$. To reject low-energy re-entrant albedo protons that could broaden and bias the energy distribution, we require the multiple scattering angle, calculated by the Kalman filter used for TKR track reconstruction, to be $<0.01$. Finally, we reject nuclear-interacting protons by selecting events with two or fewer hits in each CAL layer and no additional crystals hit in the layer containing the crystal being calibrated.

In order to determine a calibration peak shape that represents the data well but minimizes the number of free parameters, we use a two-step process. First, we produce a spectrum of path length corrected signals for all the crystals together. Each peak is fit with a Landau distribution convolved with a Gaussian, for which all parameters are left free. From this fit, we determine both the Landau and Gaussian widths, which are then fixed.

In the second step, we fit the Gaussian-convolved Landau function to spectra from each crystal separately, allowing peak position and amplitude to vary but using the fixed widths determined above. The peak most probable value (MPV) in energy units from the simulations (10.6 MeV for protons) is divided by the peak MPV in pulse height units determined by the fitting process just described to yield the desired calibration quantity for each crystal. Figure 11 shows the results, where the most probable value is the peak position.

Originally, we intended to use "heavy" GCR primary nuclei to calibrate the higher energy scales. This would be desirable since $\frac{d E}{d x}$ varies as $Z^{2}$ where $Z$ is the atomic number of the incident ion. The major difference between use of GCR heavy nuclei and protons or muons 


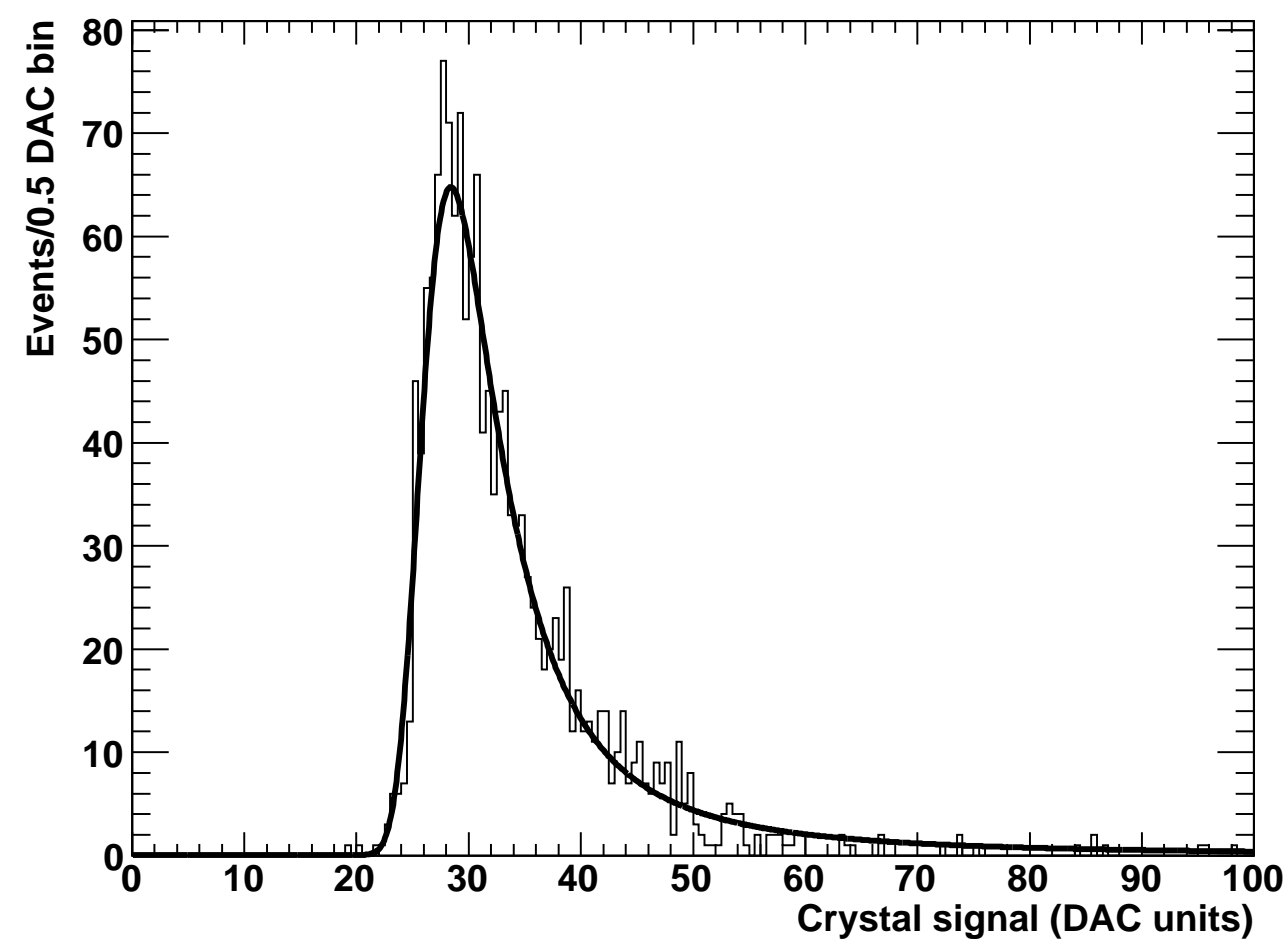

Figure 11: Energy deposited in a crystal (pathlength corrected). The position of the proton peak is given by the fit. The signal is corrected for electronics non-linearities. 

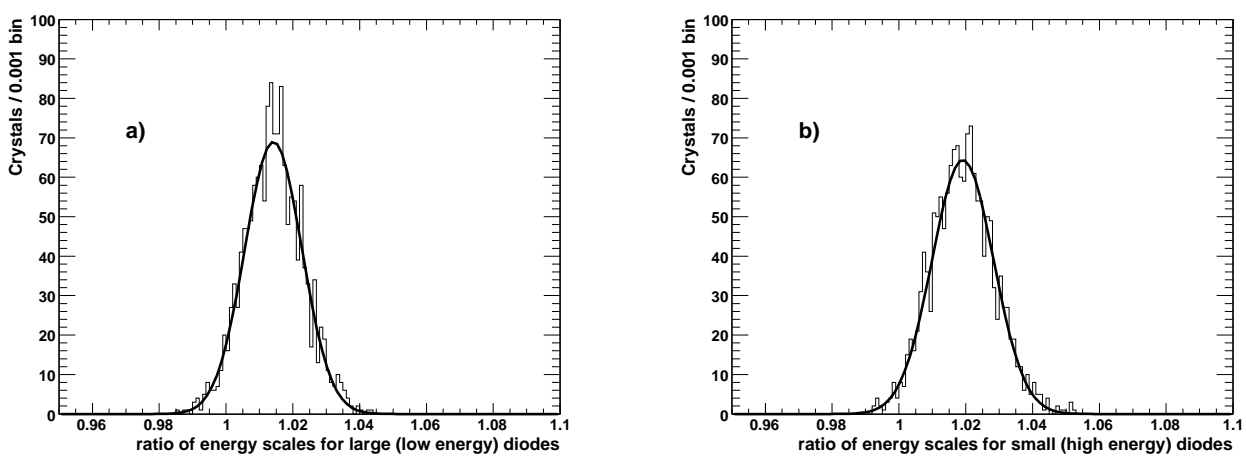

Figure 12: Ratio of the energy scales for the on-orbit calibrations performed in October and July 2008: a) low-energy diodes (mean 1.014, sigma 0.009) and b) high-energy diodes (mean 1.019, sigma 0.009).

lies in a phenomenon known as "quenching", in which the crystal light output per unit deposited energy $\left(\frac{d L}{d E}\right)$ is thought to be less for higher $Z$ nuclei than for protons or muons. In addition to being $Z$ dependent, this phenomenon also depends on the ion incident energy.

Since quenching is not well studied for the combination of $Z$ and incident energies relevant to on-orbit CAL calibration, we measured the response of the calorimeter CsI(Tl) crystals to relativistic nuclei (from carbon to iron) at the GSI facility in 2003 and 2006. The results of these studies indicated that for the ion energies examined at GSI, which were rather higher than those measured previously for $\mathrm{CsI}(\mathrm{Tl}), \frac{d L}{d E}$ was actually higher for measured nuclei with $6 \leq Z \leq 14$ than for protons [17]. Due to the lack of a physical model or understanding for this "anti-quenching" behavior, we felt that the systematic uncertainties introduced in using the heavy ion GCR calibration were unacceptable at this time and have used the proton inter-range technique instead.

In order to study any possible changes in the energy scale calibration between pre and post launch measurements and during early on-orbit operations, we calculate, for each crystal, the ratio of the energy scale measured after launch to that measured prior to launch. A Gaussian fit to this distribution leads to a mean bias, for low-energy diodes, of $\sim 1 \%$ and a standard deviation which indicates crystal-to-crystal variations of $0.8 \%$. The latter characterizes the statistical precision of this calibration procedure.

For the high-energy diodes, the bias with respect to ground calibration is $5 \%$, which is explained by the lack of any high-energy signal to reliably calibrate the ratio between the two diodes on the ground, as the muon signal is too small to be visible in the high-energy diode with flight gain settings.

The comparison of on-orbit results from July and October 2008 shows a shift of only 1-2\% and a spread, crystal-to-crystal, of $<1 \%$. The stability of our calibrations is demonstrated in Figure 12a for the low-energy diode and Figure 12b for the high-energy diode.

Despite the fact that they are not suitably well understood for absolute calibrations, we do use energy deposits from from GCR heavy nuclei $(500 \mathrm{MeV}$ for carbon nuclei and $8 \mathrm{GeV}$ for iron) for independent monitoring of the energy scale at high energies. The pathlengthcorrected spectrum shown in Figure 13 is obtained by selecting crystal hits in low-multiplicity layers, thus rejecting nuclear interactions. Narrow peaks (the carbon peak has a $5 \%$ width) 


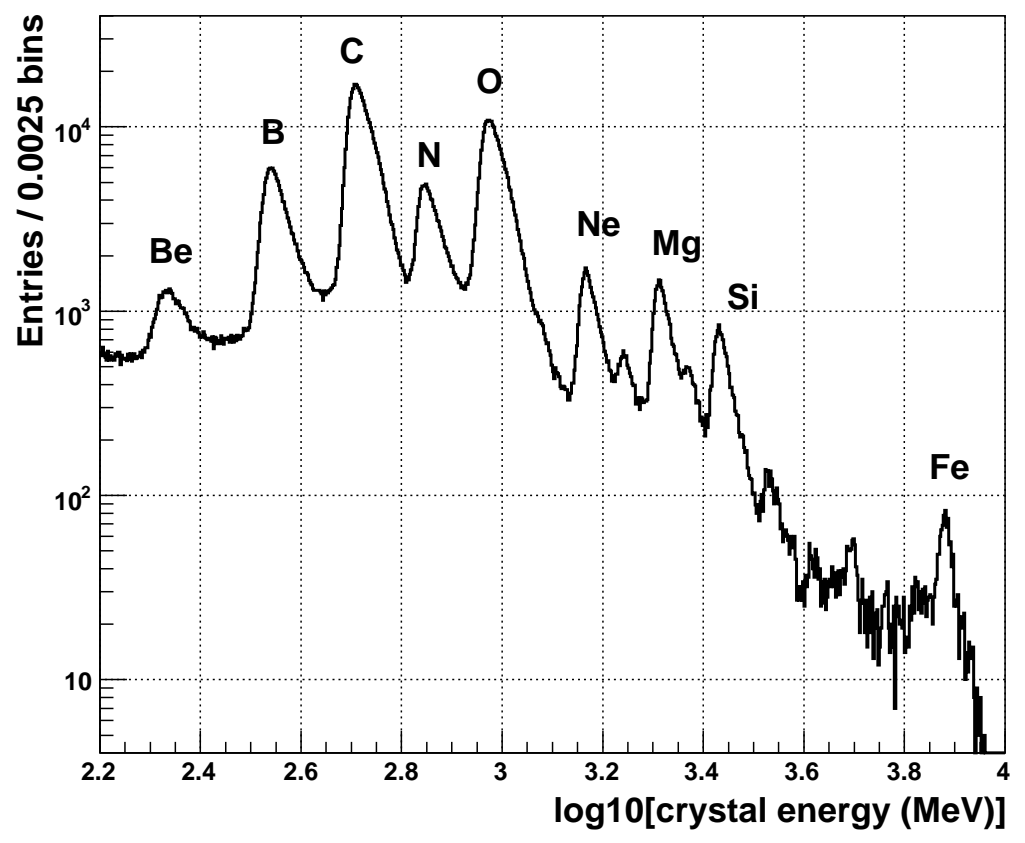

Figure 13: Energy deposited in all crystals from heavy nuclei collected during 4 days of on-orbit operations. Pathlength corrections are applied.

are easily identified. It is worth noting that the measured energies deposited by cosmic rays are consistent with the anti-quenching effect observed in the beam test data acquired at GSI. For example, the $\frac{d E}{d x}$ for carbon is observed to be about $20 \%$ higher than expected from a $Z^{2}$ scaling of the $\frac{d E}{d x}$ for protons. The count rate in the charge peaks is similar to that of the primary galactic cosmic-ray abundance, modified by loss of particles through charge-changing interactions above the CAL and by the decreasing efficiency of the on-board heavy-ion filter for higher $Z$ nuclei. For example, Figure 14 shows that, although there is a slow systematic shift, the carbon peak position is stable to within $0.5 \%$ (for the whole CAL) after 2 months of operations.

\subsection{Light asymmetry}

The design of the CAL crystals deliberately "tapers" the light propagation properties of the crystal so that we can determine the longitudinal position of an energy deposit by comparison of the signal at each crystal end. We refer to the measured quantity as the signal asymmetry, defined as the logarithm of the ratio between the signals read out at the two ends of the same crystal.

To calibrate light asymmetry, we select non-interacting heavy nuclei in a similar way to protons (see Section 5.2), but with looser TKR track quality. The TKR-determined position in a CAL crystal is obtained by extrapolating the TKR track to the center of the crystal (half-way through its thickness). Each track enters the crystal through one of the twelve evenly spaced bins defined along its length. The distribution of asymmetry signals for each 


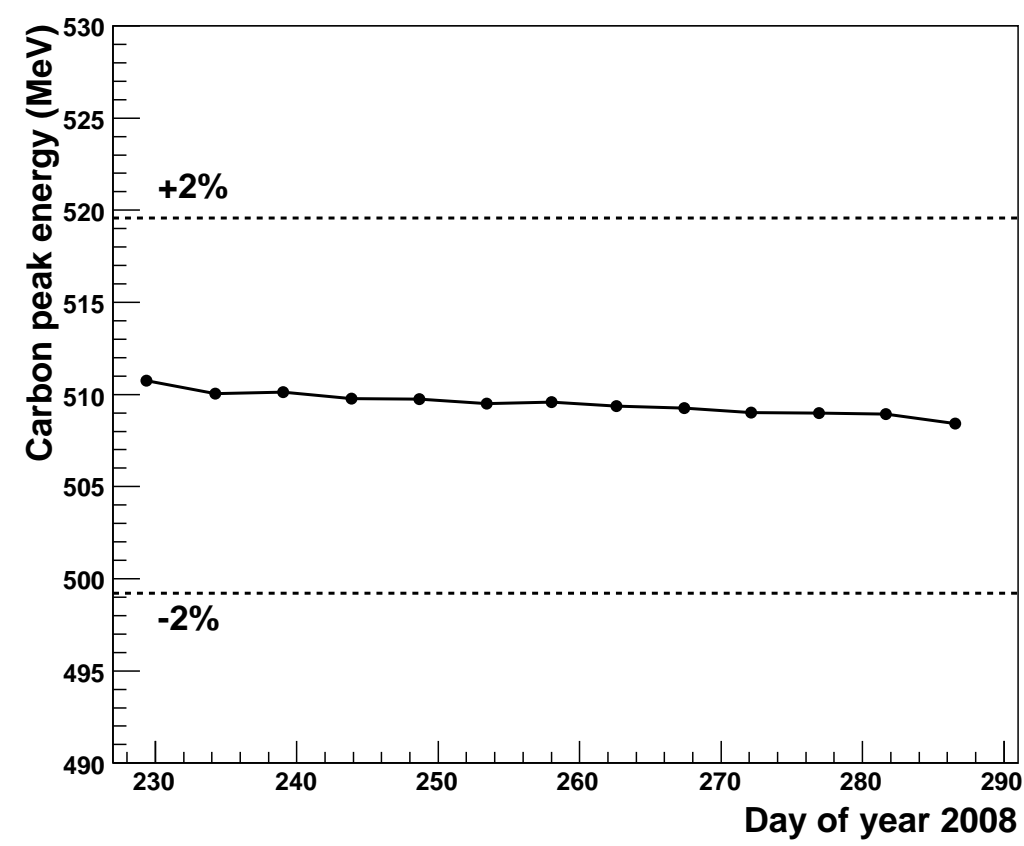

Figure 14: Position of carbon peak for 2 months of on-orbit data.

bin is collected by computing the asymmetry for each event for the appropriate bin. The first and the last bins, near the crystal ends, are not used because of known non-uniformities in light collection near the diodes. The average asymmetry is calculated for each of the ten central bins along each crystal and is fit with a spline function for purposes of interpolation.

We use the signals from the two ends of each crystal to obtain the weighted centroid of the energy depositions along the crystal. The position resolution along the crystal is defined as the RMS of the difference of the position from light asymmetry to that from track extrapolation.

We calibrated the light asymmetry of each crystal on the ground with sea-level cosmic muons, and we recalibrated on orbit with GCRs. The calibration constants derived on orbit are more precise than those derived on the ground because the GCRs suffer less multiple scattering than muons and create larger scintillation signals. The position resolution measurement for energy depositions from 200 to $900 \mathrm{MeV}$, for both LEX1, and HEX8 ranges, improves from $4 \mathrm{~mm}$ to $2 \mathrm{~mm}$ and from $13 \mathrm{~mm}$ to $9 \mathrm{~mm}$, respectively. Figure 15 shows position resolutions with pre- and post-launch constants in the energy range from 200 to 900 $\mathrm{MeV}$. Results are dominated by the carbon events which peak $~ 500 \mathrm{MeV}$. The improvement by using flight calibration constants is clearly seen.

\subsection{Trigger thresholds and upper level discriminators}

To reduce the data volume generated by the CAL and the additional dead time that would be created by moving large events through the LAT data acquisition system, each GCFE has a zero-suppression discriminator with a programmable threshold DAC. This threshold, 

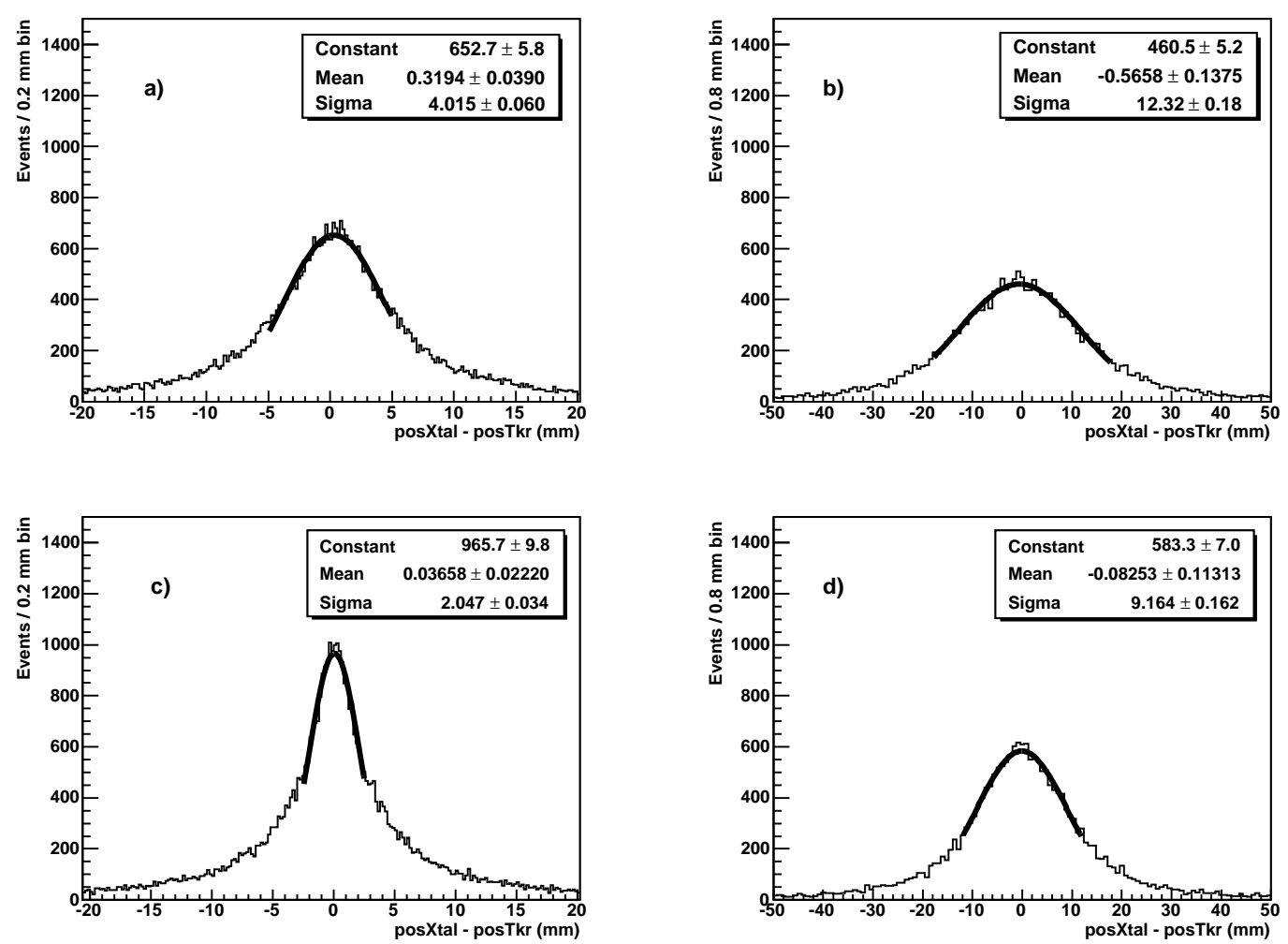

Figure 15: CAL position resolution from 200 to $900 \mathrm{MeV}$ using ground calibration constants a) LEX1 and b) HEX8, and using flight calibrations for: c) LEX1 and d) HEX8. 


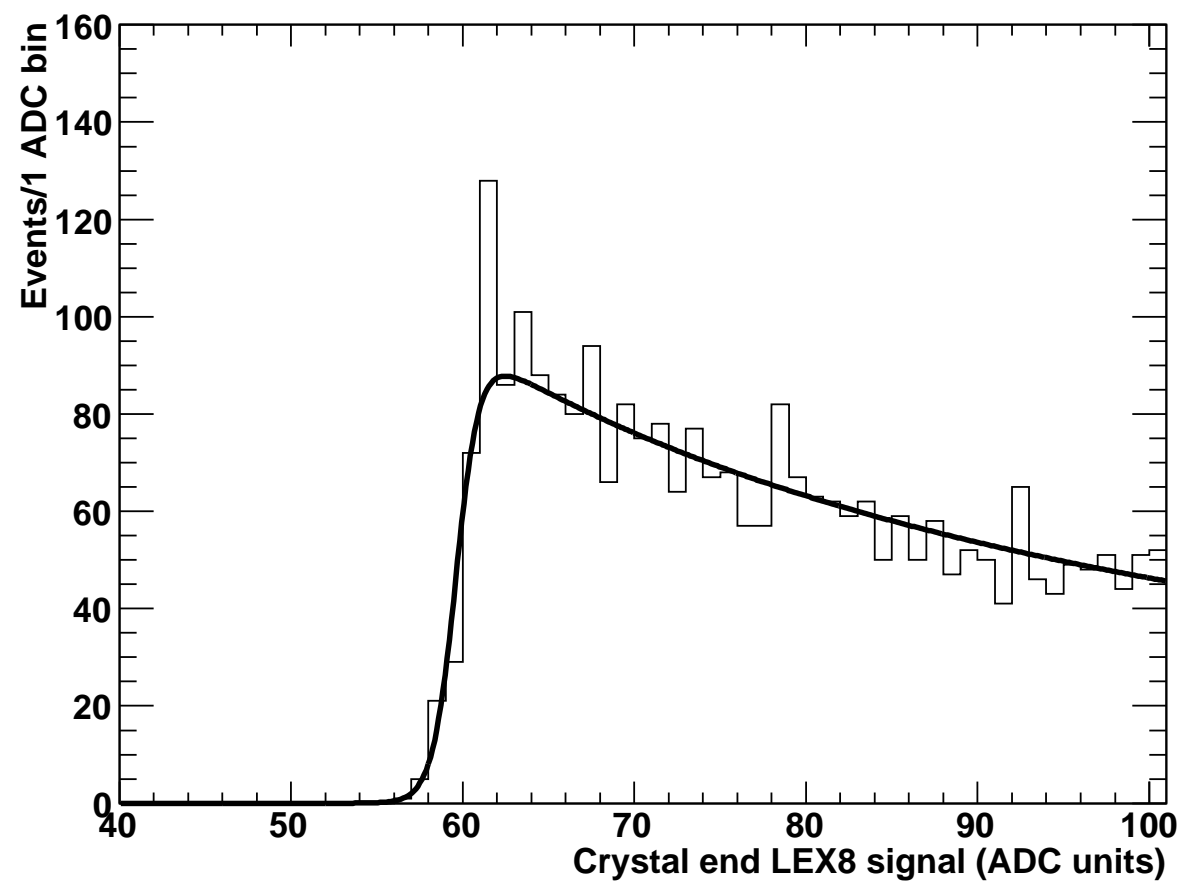

Figure 16: Signals from one end of a crystal (LEX8 range) and a fit used to determine the LAC threshold.

known as the log-accept ( $\mathrm{LAC}$ ) threshold, is nominally set to $2 \mathrm{MeV}$, which is approximately 10 times higher than the average electronic noise. The zero-suppression for an entire crystal is performed on the logical OR of the LAC discriminator states at the two ends; thus data from both ends of a crystal are included in the CAL data stream if the LAC discriminator on either end fires.

Extensive ground testing with the LAT charge injection system and sea-level cosmic ray muons established the functionality, linearity, and energy scale for each LAC discriminator. We launched with LAC settings derived from the ground calibrations, but the LAC settings are temperature dependent, primarily because the pedestal values are temperature dependent. Thus we revised the LAC calibration constants on orbit once the LAT had achieved its stable operating temperature. For each discriminator, we characterize the relationship between DAC setting and $\mathrm{LAC}$ value (in $\mathrm{MeV}$ ) with a linear model that is derived from calibration data acquired with the LAC set at two values near the nominal setting. We calibrate one end of all crystals at a time using a set of four configurations such that the LAC threshold at the crystal end not being calibrated is set to its maximum possible value, preventing it from initiating the readout, and the LAC threshold at the end being calibrated is set to the test value. This process give a LAC measurement for each channel with a statistical precision of $5 \%(\sim 0.1 \mathrm{MeV})$. Figure 16 shows an example of the signals measured at one end of a crystal (LEX8 range) and the fit that determines the LAC threshold. The values can be easily converted into energy by using results from Table 5. We are monitoring four GCFEs with out-of-family electronic noise (out of 3072 in the CAL). In February 2009, we inhibited one of the channels from participating in zero-suppression decisions because its 
noise level reached 1.5 MeV. This has no impact on the scientific performance of the CAL.

We monitor the stability of each LAC threshold in all nominal science operations data acquisitions. To measure the threshold value, we select events for which we are certain which discriminator qualified the crystal for inclusion in the data stream. Because the LAC value at each crystal end is within $10 \%$ of the setting at the opposite end, we achieve this certainty by selecting events where the signal from the two ends differs by more than $10 \%$.

The CAL provides two fast signals that participate in the formation of LAT trigger, the low energy CAL_LO and high energy CAL_HI triggers. The CAL_LO and CAL_HI trigger requests are formed as the logical OR of the outputs of the programmable fast low-energy and fast high-energy trigger discriminators, respectively FLE and FHE, at each end of a crystal. The nominal values for the FLE and FHE thresholds are $100 \mathrm{MeV}$ and $1000 \mathrm{MeV}$ per crystal, respectively, as measured at the center of the crystal by each GCFE.

Extensive testing on the ground with the LAT charge injection system clearly demonstrated the functionality and linearity of each FLE and FHE discriminator; however it gave only an approximate absolute calibration (i.e. in MeV deposited) of the threshold DACs.

The FLE and FHE thresholds are calibrated on orbit using background events recorded in dedicated-mode with additional information provided by the tower electronics module. However, this additional trigger diagnostic information only provides the logical OR of the combination of all 12 FLE or all 12 FHE discriminators for each CAL layer-end; thus it does not clearly identify which crystal end produced the trigger signal. Distinct procedures for FLE and FHE thresholds are necessary to resolve this 12 -fold ambiguity. To calibrate the FLE discriminators near the nominal $100 \mathrm{MeV}$ setting, we separately enable the trigger for each of two groups of six crystals in a layer (the six odd-numbered and the six even-numbered crystals) and require that five of the six enabled crystals have a signal below $50 \mathrm{MeV}$. Most showers share their energy between adjacent crystals in a layer, so the separation into two sets of six non-adjacent crystals readily resolves that ambiguity. The $50 \mathrm{MeV}$ energy cut ensures that only one of the six is the source of the trigger signal. To calibrate the FHE discriminators, we allow only the CAL_LO signal to initiate a LAT trigger, and we enable the FHE discriminators in two groups of six crystals per layer while we read the state of the FHE trigger diagnostic information.

The efficiency of a discriminator as a function of signal (in $\mathrm{MeV}$ ) is determined by calculating the ratio of the spectrum from events for which the discriminator fires to the spectrum for all events. As shown in Figure 17, the value of the threshold is obtained by fitting a step function to this ratio. Having established that the FLE and FHE discriminators are linear in threshold DAC setting, we calibrate each discriminator at two settings near nominal value and fit the measurements with a linear model. We calibrate FLE at $100 \mathrm{MeV}$ and $150 \mathrm{MeV}$, and we calibrate FHE at $1000 \mathrm{MeV}$ and $1500 \mathrm{MeV}$. The statistical error in determining the threshold values is $<1 \%$ for FLE and $<2 \%$ for FHE thresholds. Figure 17 illustrates how these efficiencies are obtained for the FLE and FHE thresholds for one GCFE. These data are from dedicated calibration runs taken during early operations (July 2008), when thresholds were set using calibration coefficients derived from ground tests. As it happens, the ground calibration gave threshold values for FLE and FHE somewhat higher than intended, viz. $\sim 140 \mathrm{MeV}$ and $\sim 1200 \mathrm{MeV}$, respectively. The ground FLE and FHE calibration relied on the charge-injection system, which gives pulse shapes that differ from those produced by $\mathrm{CsI}(\mathrm{Tl})$ scintillation signals. Since the trigger signal is fast $(\sim 250 \mathrm{~ns})$ and the energy 

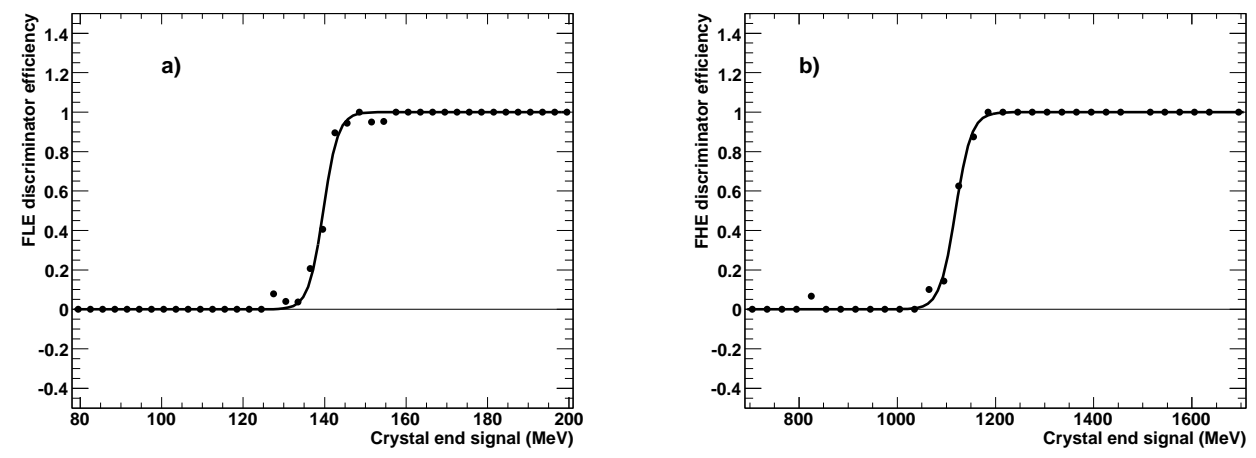

Figure 17: Efficiency versus energy for the FLE and FHE thresholds.

measurement depends on the slow shaper ( $\sim 4000 \mathrm{~ns})$, the difference in the shape of the pulses is important and creates the $40 \%$ and $20 \%$ bias we observed. We then adjusted the FLE and FHE settings using the calibration constants derived from the on-orbit calibration. We continue to monitor the FLE and FHE threshold values with data from the nominal science acquisitions.

The programmable Upper Level Discriminator (ULD) in each GCFE is responsible for switching between CAL energy ranges. To select the best range for digitization, three ULDs in each GCFE compare the outputs of three ranges (LEX8, LEX1 and HEX8) with corresponding ULD threshold (one setting per GCFE). The output of these discriminators is analyzed by the range selection logic which selects the best range as the highest range without an ULD signal [8]. All three ULD thresholds of each crystal end are defined by one DAC and are set to $\sim 5 \%$ below the saturation level of the ADC. We calibrated the ULD threshold DACs on the ground with charge injection and verified those settings and the linear calibration model on orbit during nominal science continuous acquisitions. We measured the ULDs on orbit by finding, for each energy range, the highest observed signal for each individual crystal end. Most on-orbit data-taking configurations read out only one of the four available ranges, namely the one providing largest signal below the ADC saturation, but the nominal science data explore all four ranges fully.

We updated the LAC, FLE, FHE, and ULD threshold settings after their initial on-orbit calibrations and later adjusted them to accommodate the small settling drift in pedestal values (see Section 5.1 for details). Figure 18 shows the distribution of all thresholds for all channels. The LAC threshold data from August (dashed) and November 2008 (solid) are used in Figure 18, where the effect of pedestal evolution since launch is seen as a slight broadening of the distribution. This effect is negligible for the other thresholds. The slight asymmetry in the distribution of ULD values (Figure 18d) has absolutely no effect on CAL performance; it means only that for a small fraction of channels the range switching will happen at a slightly lower energy than for the majority of channels. 

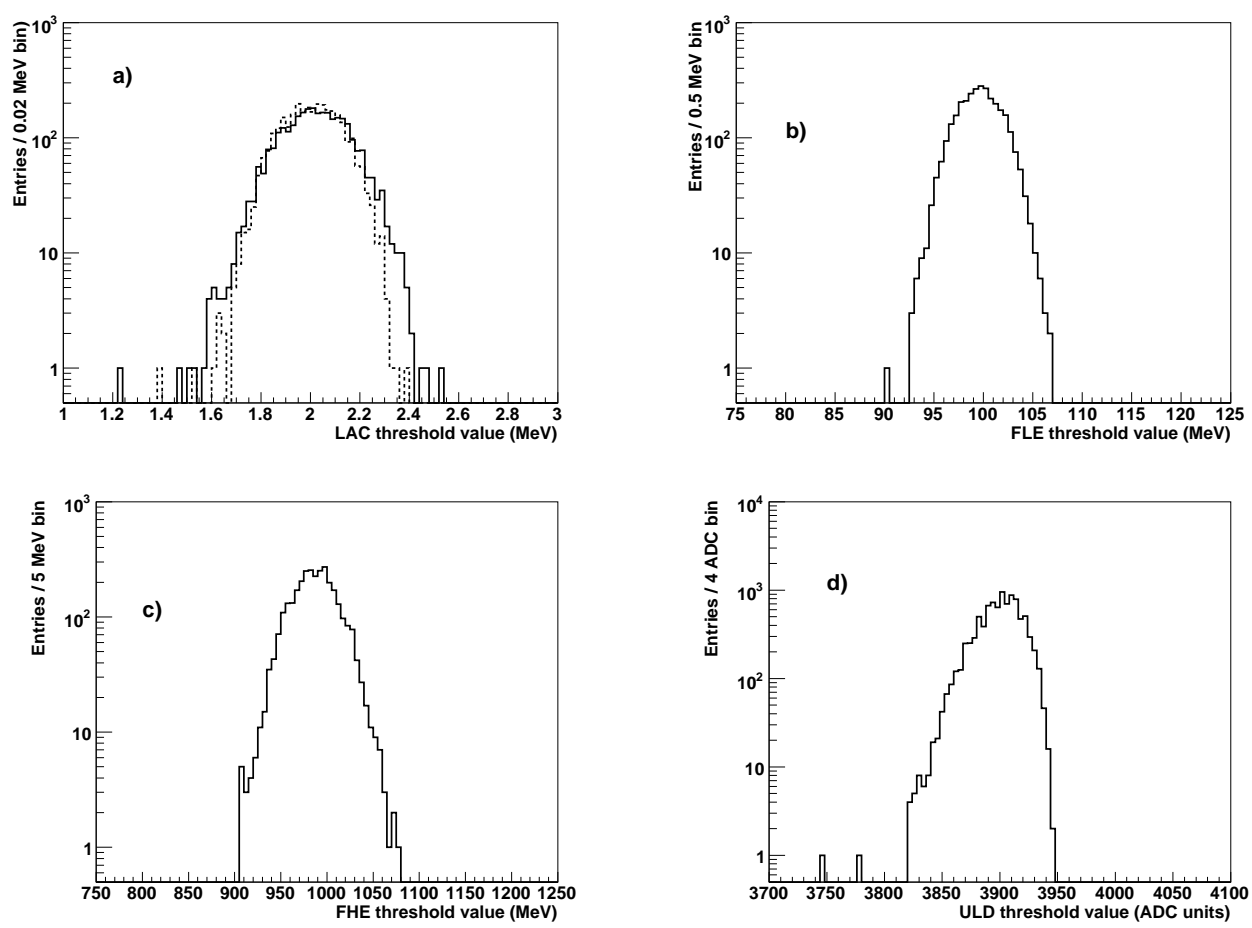

Figure 18: On-orbit measurements of threshold values for November 2008: a) LAC, b) FLE, c) FHE and d) ULD. The dashed line in the LAC histogram shows the effects of pedestal drifts seen in August 2008, prior to stabilization. The ULD thresholds are expressed in non-pedestal-subtracted ADC units. This makes it easier to judge how thresholds are set. The saturation limit corresponds to 4095 ACD units. 


\section{TKR calibrations}

The TKR is used to convert the photon to an $\mathrm{e}^{+} / \mathrm{e}^{-}$pair and to determine the incoming photon direction. It is also the main contributor to the LAT trigger. It consists of sixteen modules each composed of a stack of 19 trays. A tray is a stiff, lightweight carbon-composite panel with silicon-strip detectors (SSDs) mounted on both sides with strips oriented along the same direction. All but the three bottommost trays in each TKR module, contain an array of tungsten foils, which matches the active area of each SSD. These foils act as photon converters. Depending on their location within the tower (front or back), foils are $3 \%$ and $18 \%$ of a radiation length. Each tray is rotated $90^{\circ}$ with respect to the one above and the one below. Therefore, two consecutive trays are needed to provide orthogonal measurements of the $x, y$ coordinates. Details of the TKR design are described elsewhere [4].

Each side (top or bottom) of the tray consists of 1536 silicon strips read out by twenty four 64-channel amplifier-discriminator ASICs, GLAST Tracker Front-end Electronics (GTFE), which are controlled by two digital readout-controller ASICs, GLAST Tracker Readout Controller (GTRC). Each channel in the GTFE has a preamplifier, shaping amplifier, and discriminator similar, although not identical, to the prototype circuits described in [6]. The amplified detector signals are discriminated by a single threshold per GTFE chip; no other measurement of the signal size is made within the GTFE. The TKR electronics is discussed in detail elsewhere [7.

The trigger information is formed within each GTFE chip from a logical OR of the 64 channels. Any latched, noisy or inoperable channel can be masked. The OR signal is passed to the left or to the right, depending on how the chip is configured, and combined with the OR of the neighbor. This procedure continues down the line, until the GTRC receives a logical OR of all non-masked channels it controls. This "layer-OR" initiates a one-shot pulse of adjustable length in the GTRC, which is sent as a fast trigger signal input for the trigger decision. In addition, a counter in the GTRC measures the length of the layer-OR signal, i.e. the time-over-threshold (ToT), and buffers the result for inclusion in the event data stream. Upon receipt of a signal that acknowledges the trigger decision, each GTFE chip latches the status of all 64 channels into one of the four internal event buffers. Another 64-bit mask, which is separate from the trigger mask mentioned above, can be used to mask any subset of channels from contributing data, as may be necessary in case of noisy channels.

TKR calibrations include the determination of the noisy channels that form the trigger and data masks, of the trigger threshold settings, and ToT calibrations.

\subsection{Noisy channels}

Since noisy channels can increase the false trigger rate, and can affect instrumental dead time, ToT measurements and data volume, they are disabled in trigger and/or data masks.

The trigger mask determines the active channels that can participate in the formation of the layer-OR trigger signals. The noise occupancy for each strip is measured using not only periodic triggers but all available events, thus increasing statistics by almost 2 orders of magnitude. The computation of noise occupancy for non-periodic triggered events excludes consecutive (2 or more) layers with at least one hit in each. The occupancy measured by this method is consistent with that obtained from periodic triggers. 
Noisy channels produce off-timing trigger signals resulting in a dead time of $\sim 1 \mu$ s for the layer involved. Noisy channels also lead to incorrect ToT measurements if the noise hit occurs at the tail of the main pulse. To minimize this effect, we mask any channel with occupancy greater than $0.7 \%$. Furthermore, if the occupancy of layer-OR is greater than $8 \%$, we mask the highest-occupancy channels until we reduce it to this fraction.

The data mask determines the active channels whose data can be transmitted to the ground. Since the offline track reconstruction software is tolerant of high-occupancy channels, data mask is driven by the constraint on the data rate given by noisy channels. We mask any channel with occupancy greater than $50 \%$ since it does not carry any useful information. We limit the data size due to noisy channels to be less than $10 \%$ of TKR total data size, by requiring the average strip occupancy to be less than $5 \times 10^{-5}$, which corresponds to 44 strip hits per event. The typical strip occupancy of $\sim 2-3 \times 10^{-6}$ is dominated by accidental hits due to off-timing cosmic-ray tracks. The strip occupancy due to electronics noise is $10^{-7}$ or less. We mask highest-occupancy channels until the TKR average occupancy is reduced to $<5 \times 10^{-5}$.

The number of masked channels for trigger and data purposes was 203 before launch and changed to 206 in July 2008, to 220 in August 2008, to 284 in Octobter 2008 and finally to 316 in January 2009. These additional 113 channels are distributed across seven SSDs, while 60 of these channels are concentrated in a region of one SSD. The total number of disabled channels corresponds to only $0.04 \%$ of the total number of TKR channels.

\subsection{Trigger and data latching thresholds}

In order to minimize the noise occupancy while maximizing the hit efficiency, the nominal threshold level is set to $1.4 \mathrm{fC}(\sim 0.28 \mathrm{MIP})$. The threshold DAC value for each GTFE is calibrated using charge injection. The charge injection DAC is set to the value corresponding to $1.4 \mathrm{fC}$ and the threshold DAC is scanned (see Section6.4). The best threshold for each channel is determined by a fit to the occupancy versus threshold using the error function (integral of a Gaussian). The average threshold for each GTFE is obtained by calculating the mean value of the threshold DAC values after removing all dead and masked channels, and $5 \%$ of the channels with the largest and the smallest values.

The calibration of the threshold DAC performed after launch yields identical DAC values to those before launch for $86 \%$ of GTFEs. Only $0.04 \%$ of the total number of GFTEs (6 out of 14 000) exhibits a difference of more than one DAC value. This was already known from pre-launch measurements and it corresponded to additional noise in the system.

The GTFE data is latched following a trigger acknowledge signal, which is $0.8 \mu \mathrm{s}$ later than the typical peak of the TKR pulse shape. This value was determined using an external trigger during pre-launch tests $\mathrm{T}$. Due to the delayed data latch timing, the effective threshold for the data is different from the trigger threshold. Once the threshold DAC value is determined for all GTFEs, we measure the effective thresholds at the time of the data capture by scanning charge injection calibration DAC values. The best threshold for each channel

${ }^{\S} 0.7 \%$ corresponds to the value in which the loss in efficiency due to dead time is comparable to that from masking noisy channels.

『Although this implies that the TKR delay should be negative, the smallest allowed value is zero. This has no effect on the TKR performance. 
is determined by a fit to the occupancy versus threshold data using the error function. The ratio of data latching threshold measurements before and after launch yields a $2 \%$ shift in RMS and $0.5 \%$ shift in the mean value, which implies no significant changes from values measured prior to launch. Therefore, trigger thresholds were assumed not to have changed after launch and were not recalibrated.

Figure 19 shows the trigger thresholds obtained prior to launch and the data latching thresholds measured on-orbit. Because of the delay in latching the data, the threshold for the data capture is slightly higher and has a broader peak than that of the trigger threshold. The RMS dispersion is $\sim 5 \%$ and $\sim 12 \%$ for the trigger and data latching thresholds, respectively.

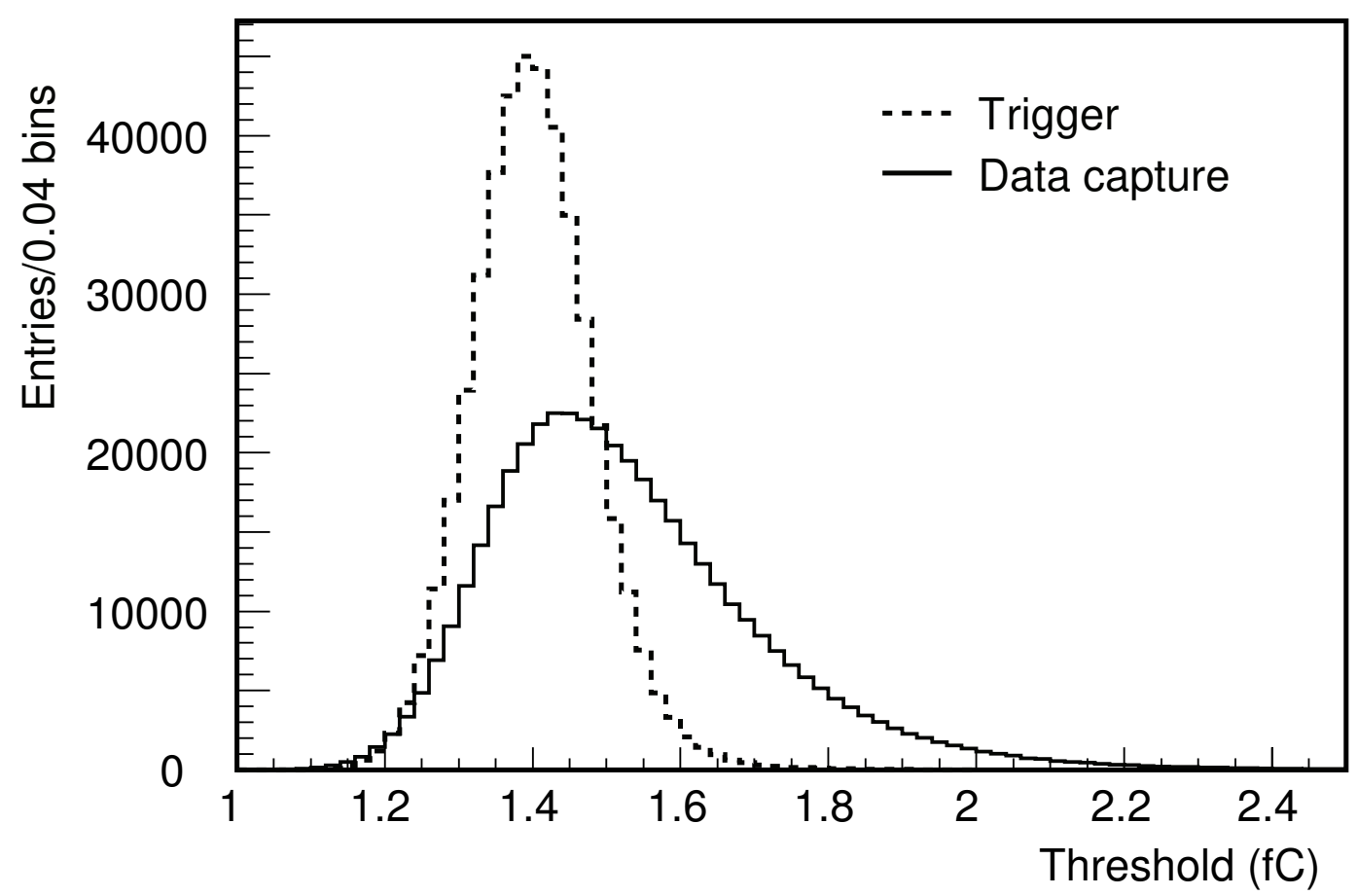

Figure 19: Effective threshold for data capture (solid) and trigger threshold (dashed).

\subsection{Time-over-threshold conversion parameters}

To determine the conversion parameters from ToT (ns) to charge deposit (fC), we measure for each channel, the ToT value for several settings of the charge injection calibration DAC. Since by definition the ToT values cannot be negative, the ToT measurements near threshold are biased toward positive values and result in slightly biased conversion parameters. Figure 20 shows the amplitude of charge injected versus ToT values, where the values for charge injection have not been corrected by the MIP scale calibration described in Section 6.4 In the fit shown in Figure 20, the ToT is described as a second order polynomial of injected charge, whose offset corresponds to the threshold value. To avoid biases near the threshold, the fit uses a fixed value for the intercept that corresponds to the calibrated threshold. The 
statistical error in the fit of order $8 \%$ and is estimated by comparing two measurements of the same curve done prior to launch. This calibration is important since the ToT gain may vary by as much as a factor of three within a GTFE. We have not performed this calibra-

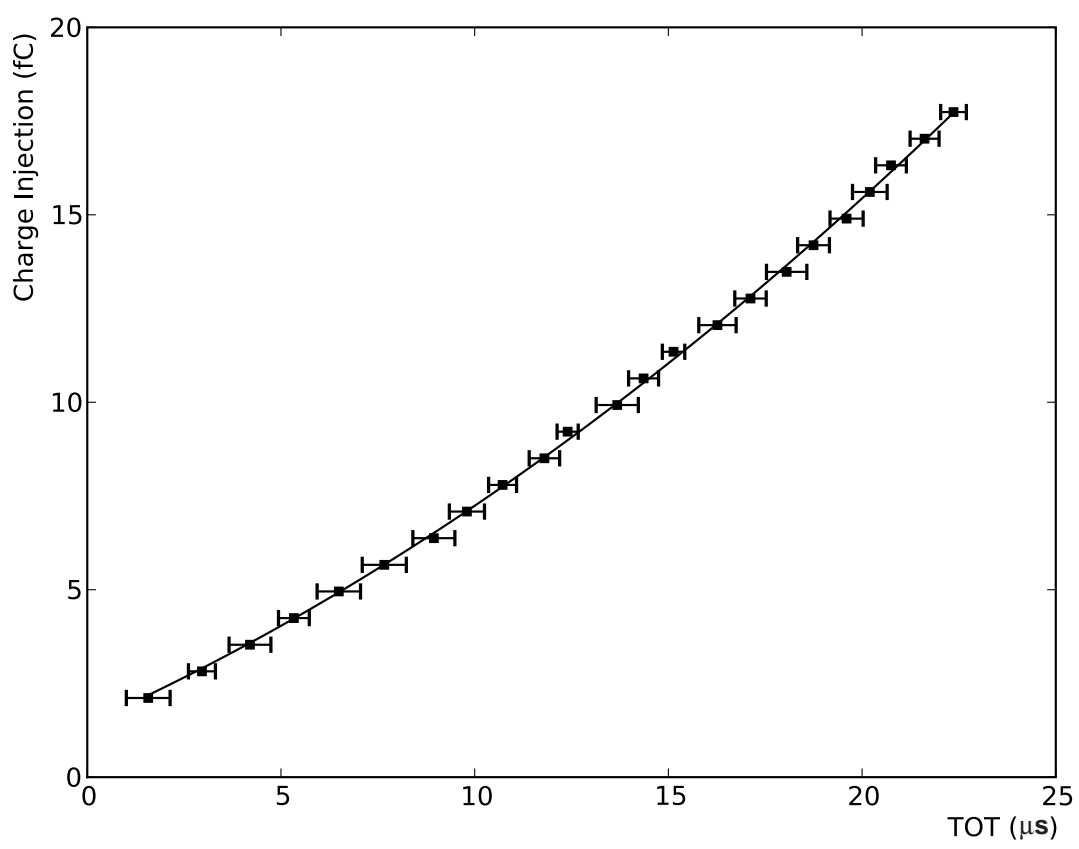

Figure 20: Amplitude of charge injected versus ToT values. The curve corresponds to a second order polynomial fit to the data.

tion after launch, since other TKR results (see Section 6.2) indicated little change in the response of TKR pulses and this calibration requires more than 10 hours of data-taking in dedicated-mode. We expect to recalibrate these values annually.

\subsection{MIP scale calibration}

The absolute calibration of the charge injection DAC was performed prior to launch using the charge deposited by surface cosmic rays. A correction factor was defined by the ratio of MIP peaks between data and Monte Carlo simulations. The distribution of this ratio shown in Figure 21 exhibits an RMS dispersion of $~ 9 \%$. The MIP deposited in each channel was calibrated using these correction factors. On-orbit, we select single-track events, close to normal incidence $(\cos \theta<-0.85)$ and with CAL energy consistent with that of a MIP. We require the RMS values of TKR hit positions with respect to the reconstructed track position to be consistent with the resolution needed to reject low-energy tracks. To avoid confusion with charge sharing between adjacent strips, we only consider layers with single hit strips. The charge deposited by a particle is corrected by taking into account its path length in the silicon. The measured ToT of the hit associated with the track is converted to charge using the conversion parameters. Following that, we fit the data for each GTFE with a Landau distribution convolved with a Gaussian. 


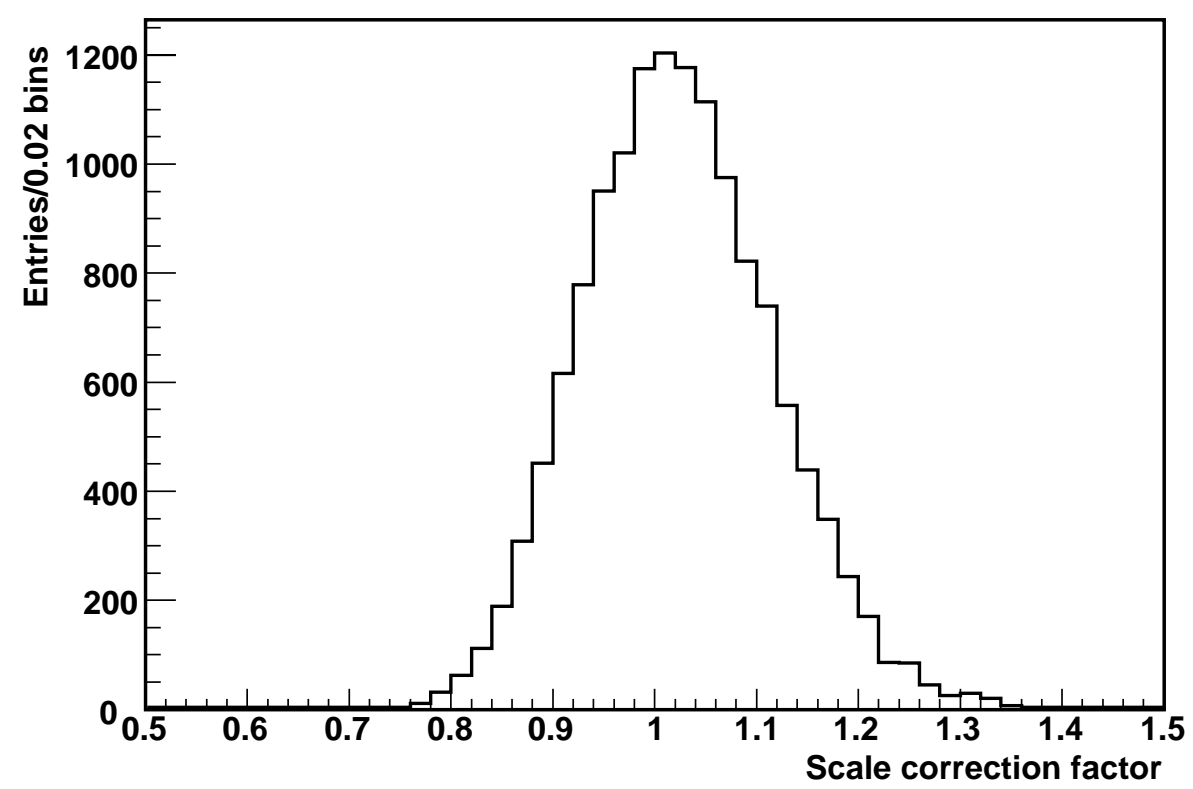

Figure 21: Measured MIP peak divided by simulated MIP peak obtained prior to launch. This charge scale correction factor is obtained for all GTFEs.

More than 10 million MIP tracks are required to accumulate sufficient entries for all GTFEs, which takes 5 days during nominal science operations. Figure 22 shows the MIP charge deposit distribution for all channels before (dotted histogram) and after (solid histogram) each GTFE is calibrated. The dispersion correction factor of $9 \%$ is included in the calibrated results.

\section{Determination of SAA polygon}

The orbit of Fermi intersects the Earth's inner radiation belt in a region which is known as the South Atlantic Anomaly (SAA). This region features geomagnetically trapped protons with energies up to hundreds of $\mathrm{MeV}$ and electrons with energies up to tens of $\mathrm{MeV}$. The flux of protons and electrons in the LAT energy range reach levels which are several orders of magnitudes above those of primary cosmic rays. This extreme particle flux imposes constraints on LAT operations. The TKR electronics saturate due to the increase in the charge deposited per live time, leading to large dead time fractions, thereby hampering scientific observations. The continuous influx of particles generates high currents in the ACD photomultiplier tubes (PMT), thus exceeding safe operating limits, which leads to slow deterioration. Therefore, during SAA passages, triggering, recording and transmission of science data are stopped and the bias voltages of the PMTs are lowered from 900V to $\sim 400 \mathrm{~V}$. Only LAT housekeeping data are recorded and transmitted to the ground.

The position along the orbit defined by the GPS receiver aboard the Fermi spacecraft determines the transition between nominal science operations and the SAA transit mode. 


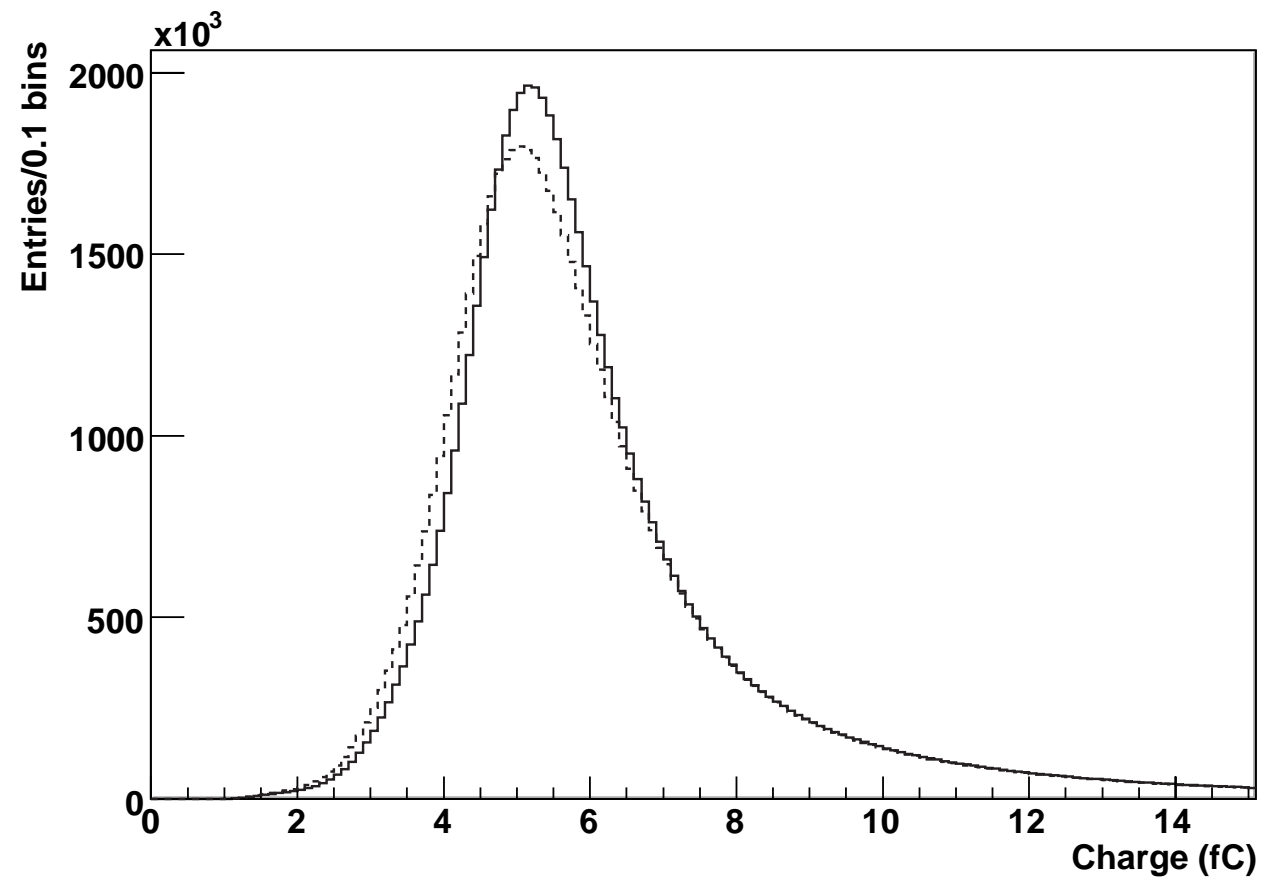

Figure 22: MIP signal (pathlength corrected) before (dotted) and after (solid) calibrations.

The latitude and longitude of the Fermi position are compared to the bounds of a polygon defined by 12 latitude-longitude vertices stored in the spacecraft memory. As the spacecraft position crosses this polygonal boundary it triggers the SAA transit mode. To avoid multiple entries and exits during a single orbit, a convex polygon is used to define the SAA region.

We chose a conservative definition for this initial SAA boundary, with the expectation that we would update the boundary based on particle rate measurements made with the LAT once it was on orbit. The first version of the polygon, or SAA boundary, was determined before launch based on models of the Earth radiation belts and data from other spacecraft. The inner radiation belt was modeled using trapped radiation models: AP-8 [18] and PSB97 [19] in conjunction with the current version of the International Geomagnetic Reference Field (IGRF-10) [20]. The 12-edge SAA boundary polygon was calculated from these models based on the contour in latitude and longitude, where the $E>20 \mathrm{MeV}$ trapped proton flux reached $1 \mathrm{~cm}^{-2} \mathrm{~s}^{-1}$. For regions where two models predictions disagreed, we chose the larger flux. The smallest convex polygon circumscribing this contour was selected and padded by a $4^{\circ}$ margin. Figure 23 a shows the trapped proton flux profiles above $20 \mathrm{MeV}$ predicted by trapped radiation models versus geographic latitude and longitude. Figure. 23b shows top scintillator count rates in the altitude range between $532 \mathrm{~km}$ and $575 \mathrm{~km}$, reported by the PAMELA experiment [21], which is similar to that of the Fermi orbit. The red polygon shows the locations of the SAA boundary edges determined before launch and used during the initial phase of the Fermi LAT mission. This definition of the SAA boundary resulted in a loss of observation time of about $17 \%$.

After launch, diagnostic data of the LAT were used to refine the size of the polygon. Even though science triggers are disabled during SAA passages, fast trigger signals remain 

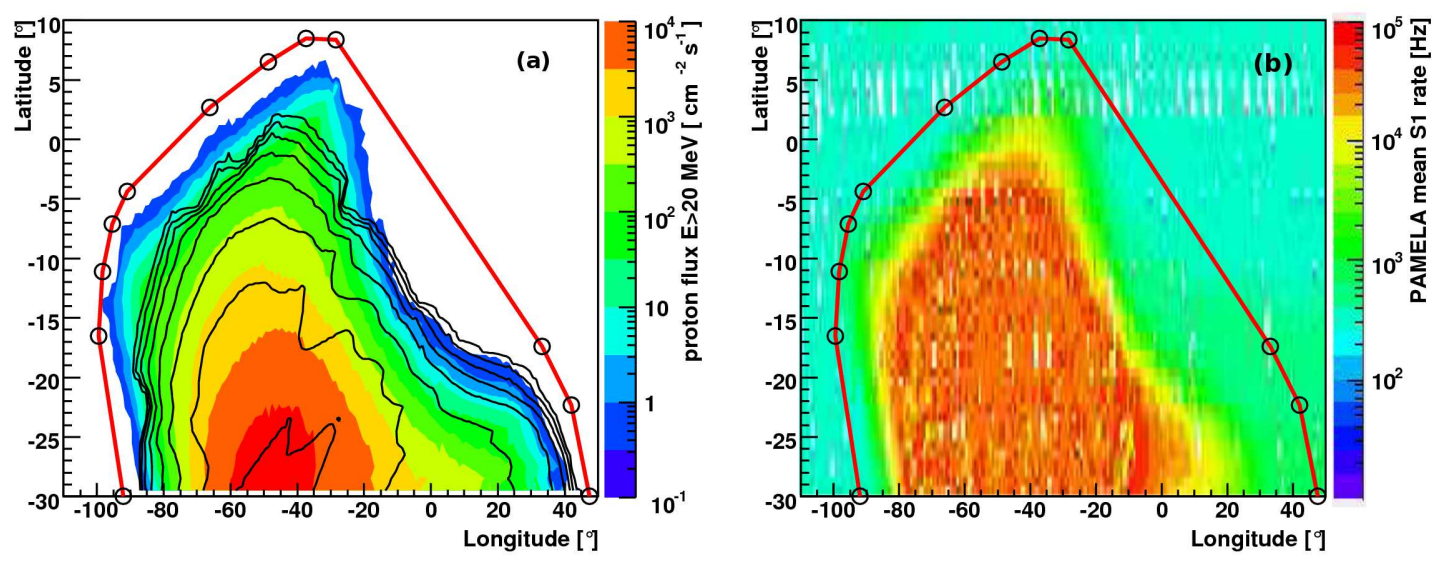

Figure 23: Trapped radiation models versus geographic latitude and longitude: a) trapped proton flux above $20 \mathrm{MeV}$ predicted by the AP-8 [18] (black contour lines) and PSB97 [19] (color contours), b) top scintillator count rates reported by the PAMELA experiment [21]. In both of the plots, the red polygon shows the locations of the SAA boundary edges determined before launch and used during the initial phase of the LAT operations.

operational. Special TKR and ACD counters can sample the rate of fast trigger signals to determine position-dependent rates of the LAT along the orbit. Figure 24 shows the rates recorded in the TKR counters versus spacecraft position. A rate increase is visible at the edges of the SAA before the TKR electronics saturates and suppress fast trigger signals, thus bringing the count rates to zero.

To define the SAA boundary using these data one has to account for particle rates and rate variations from primary and secondary cosmic rays, where both depend on the local geomagnetic cutoff rigidity at the Fermi location. Therefore, we used data from the region outside the pre-launch SAA boundary to determine these rates and set an upper limit on the expected number of cosmic-ray counts per second as a function of the local geomagnetic rigidity cutoff. The optimized SAA boundary polygon is calculated including the points that exceeded this limit. A padding of $1^{\circ}$ is applied to account for the limited resolution and sensitivity of the measurement.

Figure 24 shows the average rate of TKR counters obtained during 26.6 days of LAT nominal science operations versus geographic latitude and longitude. Superimposed are the pre-launch SAA boundary (red) used during the initial phase of the mission, and the refined polygon (yellow) uploaded to spacecraft memory. The updated polygon reduced the loss in observation time to approximately $13 \%$ of the total on-orbit time. This polygon has been the default for the LAT operations since July 28, 2008. A cross-check during nominal science operations is performed with the ACD trigger signal counters. These are more sensitive to the low-energy component $(\mathrm{E} \approx 10-60 \mathrm{MeV})$ of the trapped particle flux than those from the TKR. There is no significant increase in the rate of ACD fast trigger signals as Fermi approaches the SAA boundary, thus validating the optimized polygon.

Since the SAA moves at a rate of a few tenths of a degree per year and its size and particle fluxes vary with the solar cycle, we expect annual updates to the SAA boundary. 


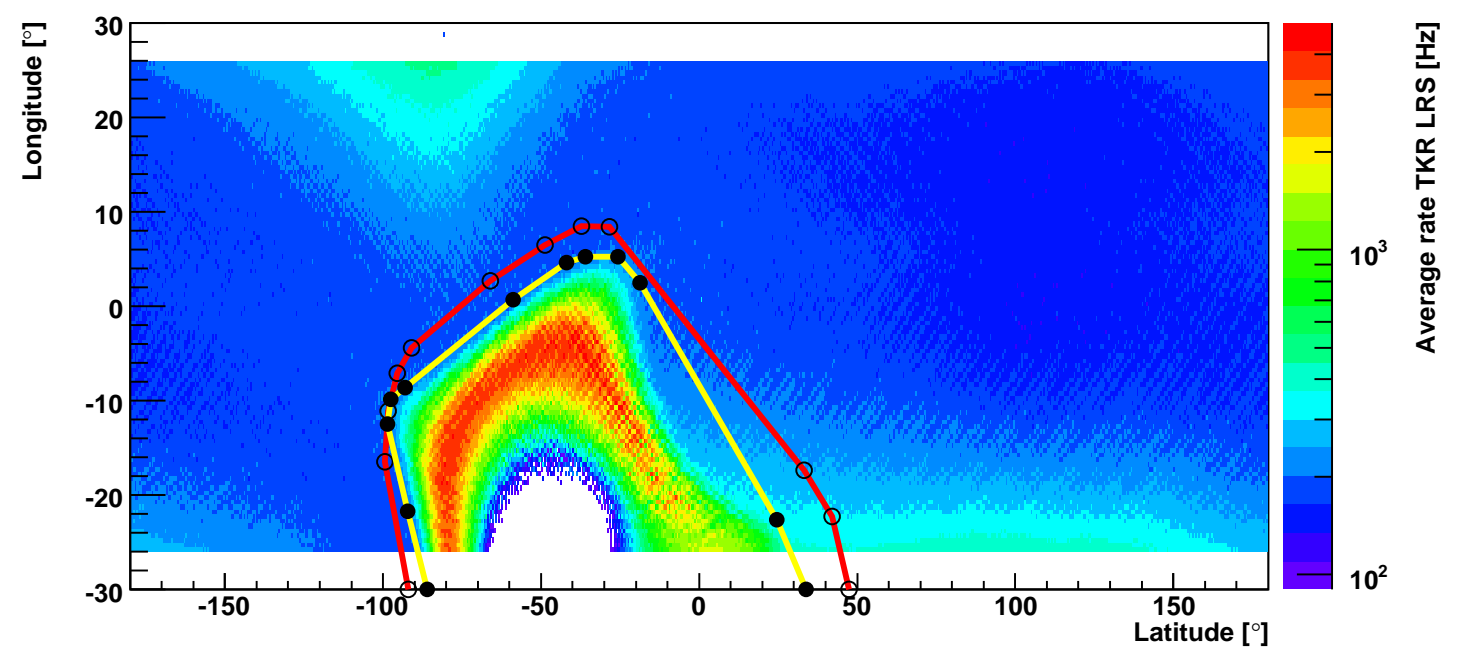

Figure 24: Average rate of TKR counters obtained during 26.6 days of LAT nominal science operations versus geographic latitude and longitude. Superimposed are the pre-launch SAA boundary (red) used during the initial phase of the mission, and the updated SAA boundary (yellow) derived from measurements of the TKR counter data (see text)

\section{Live time}

The live time is accumulated taking into account the variety of dead time effects. Science data taking is disabled during SAA passages (see Section 7). Instrumental dead time, during event latching and readout, is about $8 \%$ on average outside the SAA, although this fraction depends on the trigger rate (primarily background). The dependence on the geomagnetic latitude of Fermi is secondary. Other losses are caused by failures in transmission and ground processing and dedicated-mode calibrations.

Accurate accounting for live time is essential for obtaining calibrated fluxes and spectra for astrophysical sources of gamma rays. The live time relates the effective collecting area of the LAT to the overall exposure. Owing to the very large field of view of the LAT ( $>2 \mathrm{sr}$ ) and the relatively slow scanning rate $\left(\sim 4 \mathrm{deg} \mathrm{min}^{-1}\right)$ the accumulated live time typically is needed only coarsely ( $\sim 30 \mathrm{~s}$ intervals) for accurate exposures to be calculated. For very bright transient sources, finer accounting for live time is used, owing to the high and variable rates of triggers. For example, a bright GRB in the field of view of the LAT, such as GRB08019C [22], can induce a dead time fraction of about $16 \%$ during the impulsive phase of the burst.

The dead time for event latching and readout is tracked on-board the LAT every $50 \mathrm{~ns}$ using the $20 \mathrm{MHz}$ LAT system clock. The stability of the $20 \mathrm{MHz}$ clock is closely tracked with a 1 pulse-per-second (PPS) signal from the GPS system of the spacecraft.

During nominal science operations, the instrumental dead time is dominated by the fixed time to read out the event timing and trigger information, which imposes a minimum dead time of $26.5 \mu$ s per event. Every time the LAT triggers, further data taking is disabled until the data from the event are read out. After the end of the time coincidence window the 
latency of the trigger system is 100 ns before the new time coincidence window is available, even if the previous event was not read out. Single front-end electronic channels can be dead for several microseconds while the pulse is above threshold. In special cases, such as when the periodic trigger is enabled $(2 \mathrm{~Hz})$, the entire CAL is read out with no zero suppression and in this case, the dead time can be as large as $620 \mu \mathrm{s}$. During nominal science operations one of the trigger combinations is configured to read the CAL with all four energy ranges and zero suppression for which the dead time is about $65 \mu \mathrm{s}$.

Losses of data in transmission can effectively remove events from the data stream both directly and indirectly. The indirect effects relate to how the events are assembled in the telemetry stream. In each packet, the event times are encoded as times relative to the start of the packet. In addition, the $20 \mathrm{MHz}$ counters mentioned above roll over every 1.6 $\mathrm{s}$ and the times of the roll overs are also encoded in the packets. Loss of certain parts of a packet can cause times and live time accumulations to be lost for up to $\sim 200$ events. At the $450 \mathrm{~Hz}$ nominal event rate in telemetry, these losses can be as large as $0.4 \mathrm{~s}$. The mission specification for data loss is less than $2 \%$, and in practice loss due to transmission errors has been much less than this, in part because on-board the LAT data are retained for approximately 24 hours and retransmissions can be requested. At the ground station, Fermi telemetry is buffered for 1 week and losses in ground transmission generally can be recovered. The overall average loss has been much less than $1 \%$ to date.

Losses in ground processing of event data are also rare and are significantly less than $1 \%$.

\section{Overall timing accuracy}

Recording accurate arrival times of LAT photons is essential for studies of gamma-ray bursts and pulsar timing. Absolute timing tests were performed during pre- and post-launch activities. A discussion of LAT pulsar timing can be found elsewhere [23].

During pre-launch tests we recorded cosmic rays to measure the time difference between two GPS systems. As shown in Figure 25 a pair of scintillator tiles provided a reference for the LAT timestamps. The coincidence signal from these tiles triggered a VME-based GPS time system previously used by the ground gamma-ray telescope CELESTE. Its absolute time accuracy was previously demonstrated by measuring the Crab optical pulsar [24]. Reconstructed muon tracks traversing the LAT detector were extrapolated to their impact point on the laboratory floor and their timestamps were measured with respect to the GPS of the Fermi satellite. If a muon passed through the pair of scintillators placed next to Fermi, a GPS timestamp from a standalone VME data acquisition system was also recorded. Figure 26] shows that the LAT timestamps agreed with the reference GPS to within $0.3 \mu$ s.

GPS receivers use the arrival times of reference signals from other GPS satellites to calculate their time and position and transmit that information to processors on Fermi. This is accompanied by an electronic "Pulse Per Second" (PPS) at the moment of validity of the timestamp word. The processors, using precision oscillators on-board the spacecraft, maintain the PPS accuracy in the case of occasional short losses of GPS signal reception.

Bright gamma-ray pulsars were used to verify that the integer seconds of absolute time from the GPS receiver conform to Coordinated Universal Time (UTC), since an integer offset in the Fermi clocks would make a large shift in observed gamma-ray phase, different for each 


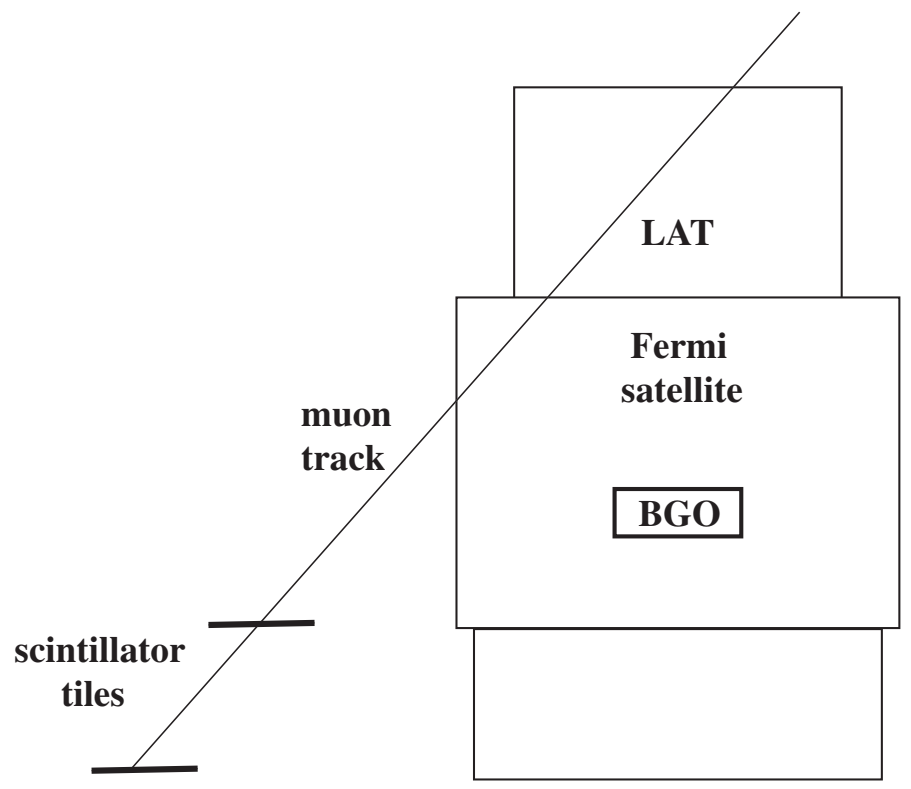

Figure 25: Diagram of the muon scintillator telescope placed next to the Fermi satellite during pre-launch tests.

pulsar. The rotational phase of the gamma-rays peaks of the Vela pulsar relative to the radio peak agree with that measured by previous experiments [25]. The first gamma-ray peak of the Crab pulsar is aligned with the radio reference, since Crab radio and gamma-ray beams appear to come from the same part of the neutron star magnetosphere [24].

The fractional part of LAT event timestamps is obtained from the counts of a $20 \mathrm{MHz}$ oscillator recorded by scalers latched at the reception of a GPS PPS signal and at the reception of an event trigger. The behavior of the oscillator was extensively characterized during the ground tests, and its frequency is recalibrated each second using the PPS-latched scaler values. On-orbit telemetry monitoring shows that the internal spacecraft timing signals behave as before launch, from which we conclude that LAT timestamps are still well within $1 \mu \mathrm{s}$ of the GPS times used by the spacecraft. GPS times are maintained within 20 ns (1 sigma) of UTC [26].

At present the best observational validation of the on-orbit clock performance comes from the pulsars PSR J0030+0451 and PSR J1939+2134. The peak width of $<120 \mu \mathrm{s}$ reported for PSR J0030+0451 in [27] demonstrates the stability of the LAT event times over six months of data-taking, but not their absolute accuracy. However, the $1.56 \mathrm{~ms}$ pulsar PSR J1939+2134 appears to have a gamma peak aligned with the radio peak to better than $1 / 20$ of a rotation of the neutron star, that is, $80 \mu \mathrm{s} \mathrm{[28].} \mathrm{This} \mathrm{translates} \mathrm{to} \mathrm{an} \mathrm{absolute}$ time accuracy if one assumes that both the gamma and radio emission come from the same region in the pulsar's magnetosphere. 

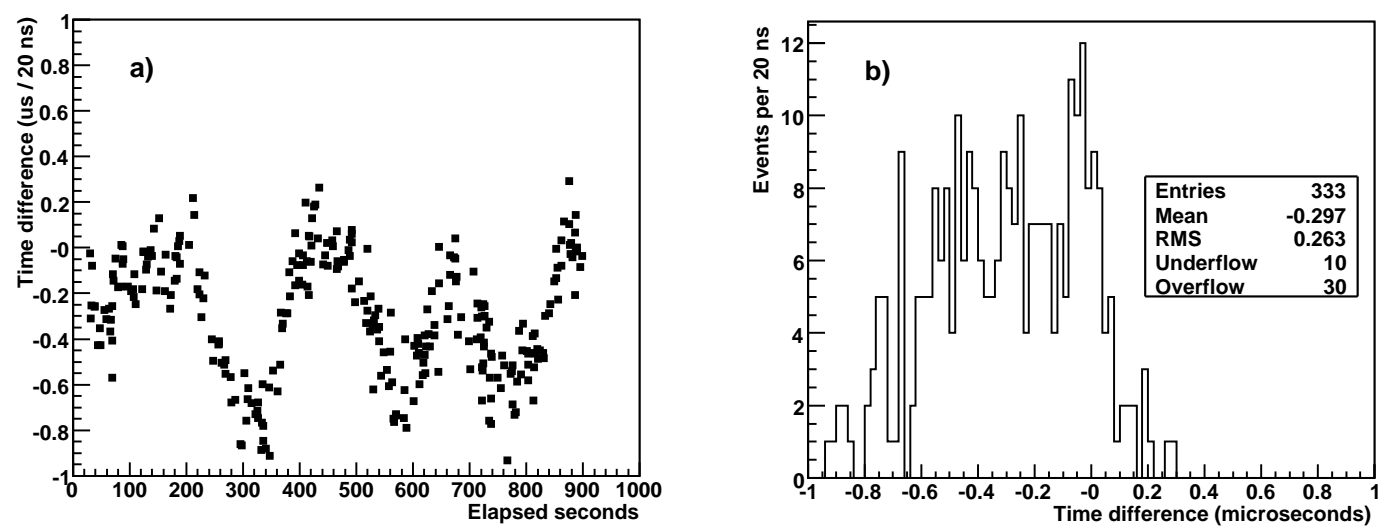

Figure 26: Results from pre-launch tests: a) differences in the times recorded with the two acquisition systems, versus elapsed time; b) histogram of time differences indicating mean and RMS values around $0.3 \mu \mathrm{s}$.

\section{Internal and spacecraft boresight alignments}

The accuracy of the directions of reconstructed events dependes on the knowledge of the exact position of each element of the TKR, i.e individual SSD or planes. Misalignments of any element can broaden the instrument response function, thus lowering the LAT sensitivity. We perform the following alignment procedures:

1. intra-tower alignment to determine the position and orientation of each element of a single tower with respect to an ideal coordinate system;

2. inter-tower alignment to determine the position and orientation of each tower with respect to an ideal coordinate system;

3. spacecraft alignment to determine the rotation of the LAT with respect to the Fermi on-board guidance, navigation and control system.

\subsection{Intra-tower alignment}

As described in Section 6, each TKR tower consists of 36 silicon planes each instrumented with sixteen silicon microstrip detectors. All strips in a plane are oriented along the same direction. The intra-tower procedure aligns the planes horizontally (along the measured coordinate) and vertically (perpendicular to the silicon plane), and determines all rotations of the planes within the TKR tower.

Due to the procedures for construction and assembly of towers, we expect only minor deviations from the original positions. This simplifies the formalism used for the alignment, and we assume first-order approximations to deviations.

The trajectory of a particle traversing the TKR is characterized by a reconstructed track, which consists of a list of associated silicon strip hits joined by a straight line. As the particle 


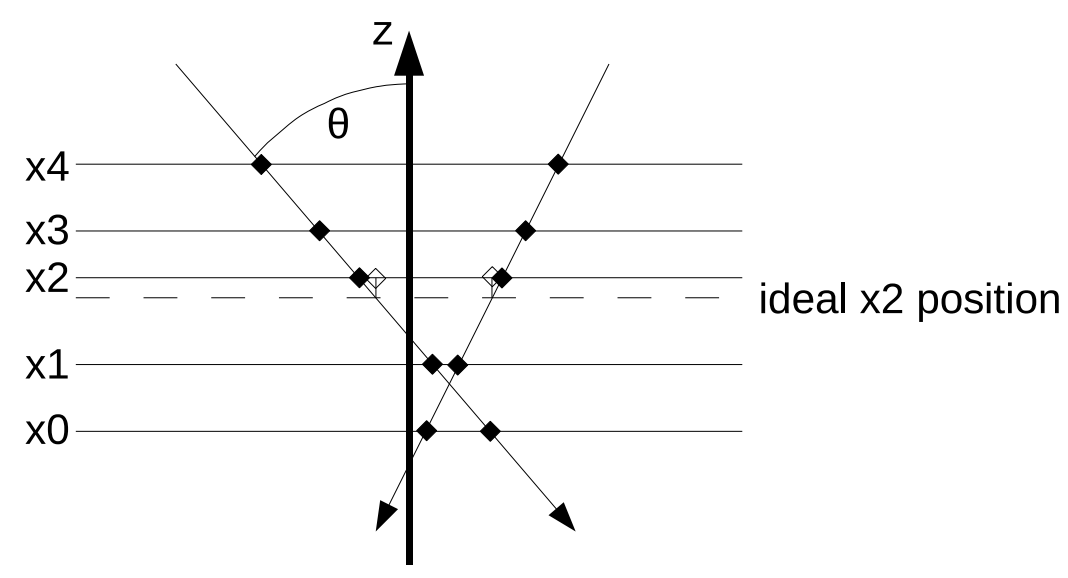

Figure 27: The diagram shows five silicon layers ( $\mathrm{x} 0$ to $\mathrm{x} 4$ ) and the ideal $\mathrm{x} 2$ layer, crossed by two tracks. Measured hits are denoted as filled diamonds, extrapolated hits (assuming the ideal position of the $\mathrm{x} 2$ plane) as open diamonds. The residual is composed of two contributions: $\Delta x$ due to horizontal misalignment, and $\Delta z \cdot \tan (\theta)$ due to vertical misalignment.

encounters material in its path its direction is affected by multiple scattering. The effect is more pronounced in the presence of dense materials such as the tungsten converter foils. Thus, a fit to an otherwise straight track results in deviations of the real hit positions from an ideal straight track referred to as residuals. The diagram in Figure 27 shows two tracks in the $x z$ plane and illustrates how residuals relate to measured and ideal positions. A distribution of residuals should be centered at 0 , and any deviation from zero is an indication that the element studied is not at its assumed position. Figure 28 illustrates how straight tracks are used to determine the horizontal and vertical misalignments. It shows the hit residuals versus $\tan (\theta)$ (i.e. inverse of the slope) of the track, for one silicon plane (arbitrarily chosen). A straight line fit through these points produces an offset and a slope, which correspond to deviations of the real position from the assumed one. Results from the fit determine horizontal $(\Delta x)$ and vertical $(\Delta z)$ deviations, corresponding to shifts along and perpendicular to the strip coordinates, respectively.

Intra- and inter-tower TKR alignments are iterative processes that use reconstructed events recorded during nominal science operations with no requirement on any on-board filter. As a result, each TKR element is subject to a wide range of event types, thus minimizing the possibility of selection bias.

Here we briefly describe a method common to both intra- and inter-tower alignments. The event reconstruction associates hits to tracks and classifies them according to their track length and straightness. The internal TKR alignment procedures benefits from track reconstruction by using only the first (best) track in the event, i.e. all other tracks are discarded. The list of the hits is then input to the alignment algorithm.

The alignment algorithms rely on two important pieces: the raw position information, i.e. the plane and strip containing a hit, and the smoothed position, which results from track finding and optimization through the Kalman filter algorithms [1]. Since the raw position cannot be used to determine the location of the hit along the strip, the algorithm uses the 


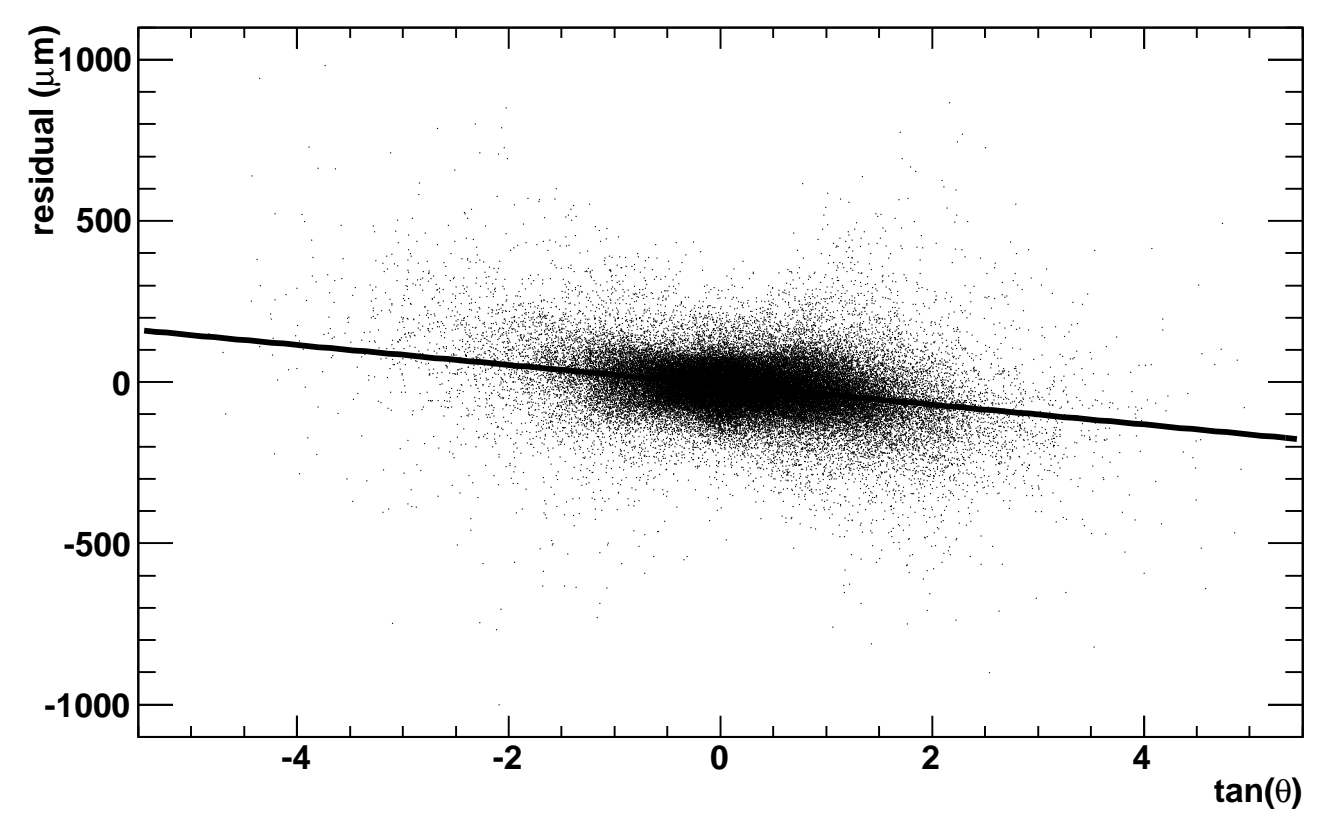

Figure 28: Residual versus $\tan (\theta)$ of the track, for a silicon plane arbitrarily chosen. A fit with a straight line provides an offset and a slope, which correspond to deviations of the real position to the assumed one.

corresponding smoothed position, instead. Finally the algorithm fits the hit positions with a straight line, computes the $\chi^{2}$ of the fit, and evaluates the residuals, and the angle $\theta$ of the track with respect to the tower axis.

Correlations similar to those depicted in Figure 28 are used to determine all alignment parameters listed in Table 6. Note that the angle $\theta$ is always measured in the coordinate of the silicon plane (e.g. $\theta_{x}$ for $x$-planes). The sign of the correlation for $\operatorname{rot} z$ is different for the $x$ and the $y$-planes. There are six parameters to determine, but only four correlations. Each correlation gives two parameters: the horizontal position, either $\Delta x$ (for $x$ planes) or $\Delta y$ (for $y$ planes), and $\Delta z$ or any of the three rotations. For each TKR element the iterative

\begin{tabular}{|c|c|}
\hline Correlation of track parameters & Alignment Parameters \\
\hline residual versus $\tan (\theta)$ & $\Delta h+\Delta z$ \\
\hline residual versus $x \cdot \tan (\theta)$ & $\Delta h+\operatorname{rot} x$ \\
\hline residual versus $-y \cdot \tan (\theta)$ & $\Delta h+\operatorname{rot} y$ \\
\hline residual versus $\mp$ position along the strip & $\Delta h+\operatorname{rot} z$ \\
\hline \hline
\end{tabular}

Table 6: Correlations of track parameters, and resulting alignment parameters. $\Delta h$ is the measured horizontal coordinate of a plane, i.e. $\Delta x$ for $x$-planes and $\Delta y$ for $y$-planes. See text for more details.

algorithm determines five parameters that are measured relative to other elements whose positions are also unknown. The knowledge of the precise positions and rotations increases with successive iterations, and each parameter converges to its correct value. The decision 
for convergence comes from comparing the largest deviation of any positional (rotational) parameter between two successive iterations and by requiring the maximum deviations to be $0.01 \mu \mathrm{m}(1 \mu \mathrm{rad})$. Convergence typically occurs after 100 iterations. Currently, the smallest elements considered in intra-tower alignment are single silicon planes (top and bottom of trays as defined in Section [6). In the future, we will extend the method to align SSD's.

Table 7 shows the results from the on-orbit intra-tower alignment of 576 TKR planes. Offsets and slopes (as in Figure 28) are entered in a histogram and the standard deviation $(\sigma)$ of each distribution is shown in Table 7 . Since planes are aligned with respect to an ideal frame, by construction, the sum of all shifts (for all parameters) average to the mean value of 0 . Results clearly demonstrate the quality of the assembly of individual towers since standard deviations are within $\pm 61 \mu \mathrm{m}$ and $\pm 220 \mu \mathrm{rad}$.

\begin{tabular}{|c|c|}
\hline Parameter & Standard deviation $(\sigma)$ \\
\hline$\Delta \mathrm{x}$ & $\pm 43 \mu \mathrm{m}$ \\
$\Delta \mathrm{y}$ & $\pm 59 \mu \mathrm{m}$ \\
$\Delta \mathrm{z}$ & $\pm 61 \mu \mathrm{m}$ \\
$\operatorname{rot}_{x}$ & $\pm 220 \mu \mathrm{rad}$ \\
$\operatorname{rot}_{y}$ & $\pm 220 \mu \mathrm{rad}$ \\
$\operatorname{rot}_{z}$ & $\pm 210 \mu \mathrm{rad}$ \\
\hline
\end{tabular}

Table 7: On-orbit intra-tower alignment constants, for 576 planes (averaged for all towers).

\section{$10.2 \quad$ Inter-tower alignment}

The inter-tower alignment procedure aligns towers spatially and determines their rotations with respect to the LAT reference frame, and is performed only after the intra-tower alignment constants have been determined. While the latter uses the residuals of each hit to determine the alignment constants, the former relies on track segments of events that cross tower boundaries. We determine the orientation of each tower by evaluating the angles between pairs of track segments. The rotation in one axis is given by the scalar product of the track segments, projected on the plane spanned by the two other axes, and averaged over all selected events:

$$
\cos \left(\operatorname{rot}_{i}\right)=\left\langle\sum \frac{\vec{t} \overrightarrow{t^{\prime}}}{|t|\left|t^{\prime}\right|}\right\rangle,
$$

where $\vec{t}$ and $\overrightarrow{t^{\prime}}$ are the track segments in vectorial form and $i$ corresponds to $x, y$, or $z$. For convenience, Eq.(1) can be rewritten in terms of track slopes $\cot (\theta)$, yielding

$$
\operatorname{rot}_{i}=\left\langle\sum \frac{\Delta \cot \left(\theta_{i}\right)}{1+\cot \left(\theta_{i}\right)^{2}}\right\rangle
$$

After fixing rotations, each pair of track segments is projected onto a reference plane between both towers, as depicted in Figure 29. Each intersection defines a position on this plane. For perfect alignment, these positions are identical. The differences between both positions are analyzed as in Section 10.1, i.e. by searching for correlations between the 


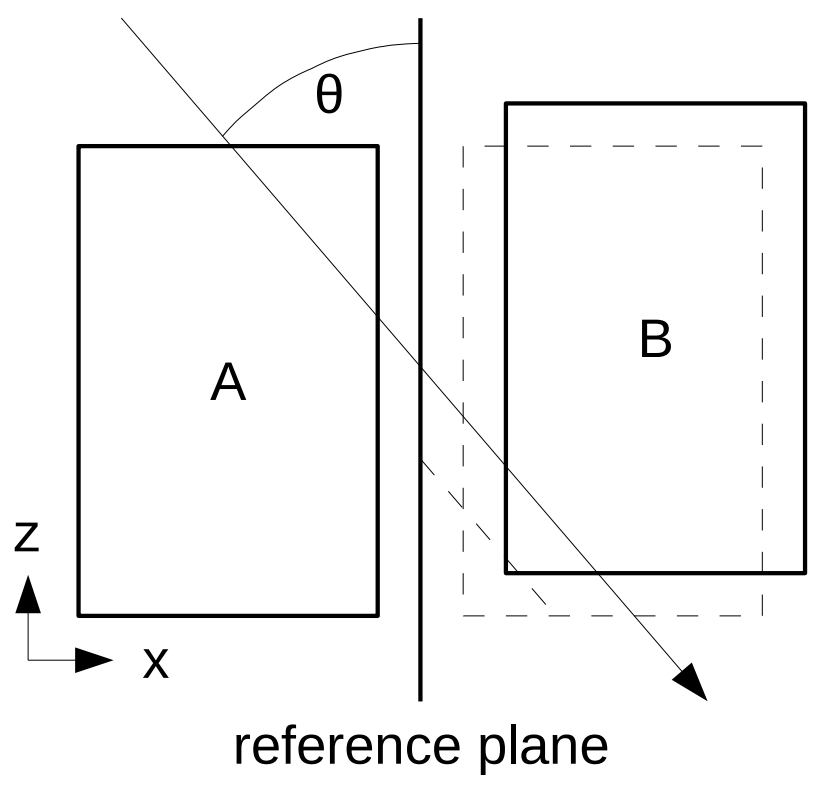

Figure 29: Horizontal and vertical alignment for a pair of towers. the real positions are drawn with solid lines, the assumed position of tower B with dashed ones. The black line between the towers marks the reference plane. The real particle track is shown with solid line, the extrapolated track based on the assumed position dashed.

position differences and $\cot (\theta)$ of the track. In the example of Figure 29, the residual has a $\Delta \mathrm{z}$ and a $\Delta \mathrm{x} \cdot \cot (\theta)$ contribution due to vertical and horizontal misalignments.

Table 8 summarizes the results from the on-orbit inter-tower alignment of 16 TKR towers. Contrary to the intra-tower alignment, only a small number of values (16 towers instead of 576 planes) are available to determine the $\sigma$ of the distribution for each of the alignment parameters. Because of that, Table 8 has an additional column that shows the largest and smallest value for each parameter. Results clearly demonstrate the quality of the assembly of towers in the LAT since standard deviations are within $\pm 120 \mu \mathrm{m}$ and $\pm 260 \mu \mathrm{rad}$. Alignment

\begin{tabular}{|c|c|c|}
\hline Parameter & Values (mix,max) & Standard deviation $(\sigma)$ \\
\hline$\Delta \mathrm{x}$ & $(-250,+190) \mu \mathrm{m}$ & $\pm 119 \mu \mathrm{m}$ \\
$\Delta \mathrm{y}$ & $(-90,+130) \mu \mathrm{m}$ & $\pm 68 \mu \mathrm{m}$ \\
$\Delta \mathrm{z}$ & $(-150,+150) \mu \mathrm{m}$ & $\pm 87 \mu \mathrm{m}$ \\
$\operatorname{rot} x$ & $(-450,+400) \mu \mathrm{rad}$ & $\pm 260 \mu \mathrm{rad}$ \\
$\operatorname{rot} y$ & $(-360,+480) \mu \mathrm{rad}$ & $\pm 250 \mu \mathrm{rad}$ \\
$\operatorname{rot} z$ & $(-360,+360) \mu \mathrm{rad}$ & $\pm 230 \mu \mathrm{rad}$ \\
\hline
\end{tabular}

Table 8: On-orbit inter-tower alignment constants for 16 towers.

procedures were validated using a simulated dataset of cosmic protons, generated with randomly misaligned geometry for the LAT. Figure 30 shows the results when the alignment procedure is applied to data acquired before and after launch. Here the standard deviations $\left(\sigma_{\Delta}\right)$ are calculated from a histogram of differences between ground and on-orbit measurements. Figure 30 a shows the correlation for intra-tower positional alignment constants, while 

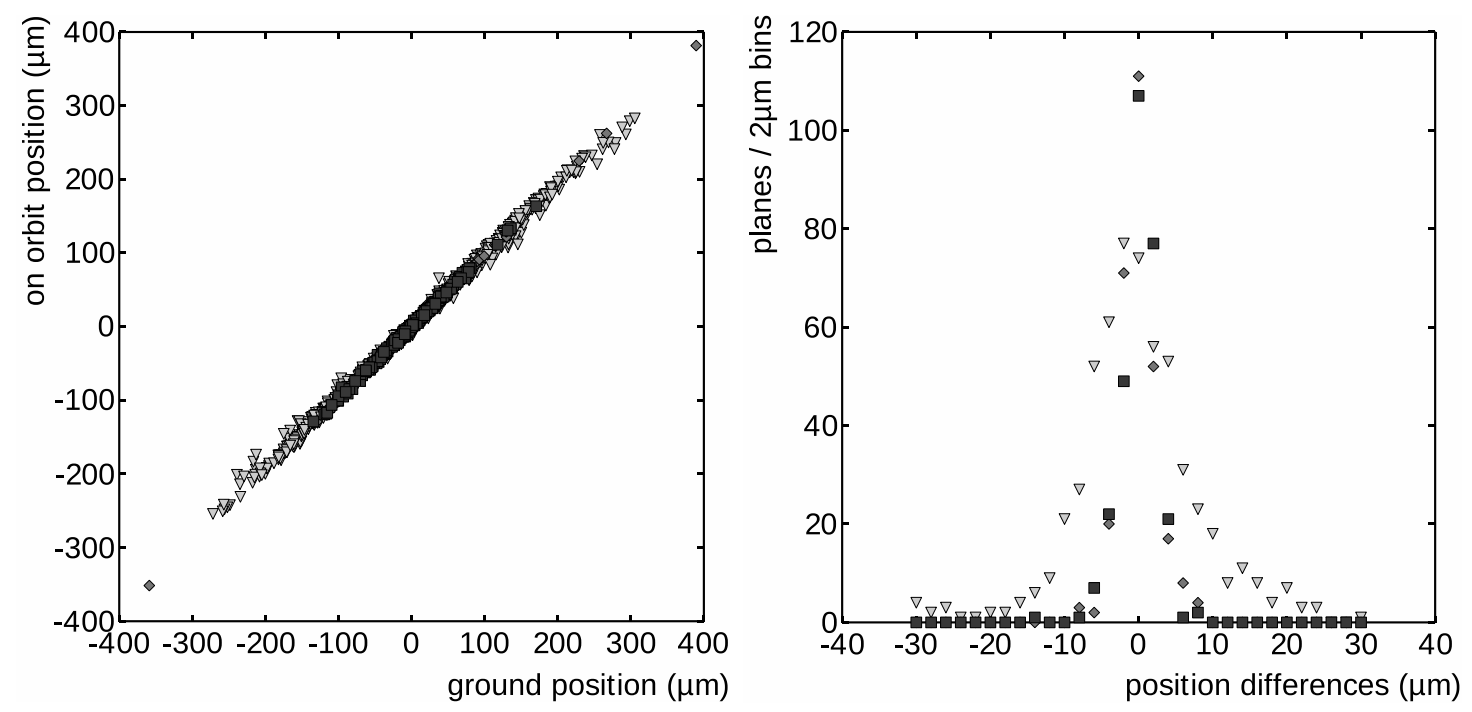

Figure 30: On-orbit data: a) correlation between ground and on-orbit inter-tower positional alignment constants, b) the histogram of the differences $(\Delta \sigma)$, where $x, y$ and $z$ correspond to squares, diamonds and triangles, respectively.

Figure 30b displays the histogram of these residual differences. Table 9 compares unbiased datasets obtained on ground ( $\sim 8$ million surface muons) with orbit data ( $\sim 20$ million events, mostly cosmic protons) and lists the average differences for both intra- and inter-alignment,

\begin{tabular}{|c|c|c|}
\hline Parameter & $\sigma_{\Delta}$ (intra-tower) & $\sigma_{\Delta}$ (inter-tower) \\
\hline$\Delta \mathrm{x}$ & $2.4 \mu \mathrm{m}$ & $30 \mu \mathrm{m}$ \\
\hline$\Delta \mathrm{y}$ & $2.5 \mu \mathrm{m}$ & $32 \mu \mathrm{m}$ \\
\hline$\Delta \mathrm{z}$ & $8.8 \mu \mathrm{m}$ & $17 \mu \mathrm{m}$ \\
\hline $\operatorname{rot} x$ & $51 \mu \mathrm{rad}$ & $53 \mu \mathrm{rad}$ \\
\hline $\operatorname{rot} y$ & $53 \mu \mathrm{rad}$ & $42 \mu \mathrm{rad}$ \\
\hline $\operatorname{rot} z$ & $36 \mu \mathrm{rad}$ & $24 \mu \mathrm{rad}$ \\
\hline
\end{tabular}

Table 9: Standard deviations $\left(\sigma_{\Delta}\right)$ of the differences between ground and on-orbit alignments. Inter-tower values are averaged over all three positions and rotations.

for all parameters. Results from Figure 30 and Table 9 demonstrate that misalignments due to launch or temperature variations on-orbit are small and limited to within $\pm 35 \mu \mathrm{m}$ and $\pm 55 \mu \mathrm{rad}$.

Finally, Figure 31 shows all correlations between the rotation angles obtained for each tower using on-orbit data acquired by the end of October 2008. Tower numbers are labeled from 0 to 15. Squares denote the correlation between $\operatorname{rot} x$ ( $x$-axis) and roty (left $y$-axis label), and diamonds between $\operatorname{rot} x$ and $\operatorname{rot} z$ (right $y$-axis label). The spread in the correlation between $\operatorname{rot} x$ and rot $y$ translates directly into a pointing inaccuracy on the celestial sphere, e.g. a misalignment of $0.3 \mathrm{mrad}$ in $\operatorname{rot} x$ or rot $y$ for one tower would cause a source position error of the same size. The absence of clustering in this distribution indicates that there are no obvious systematic effects. 


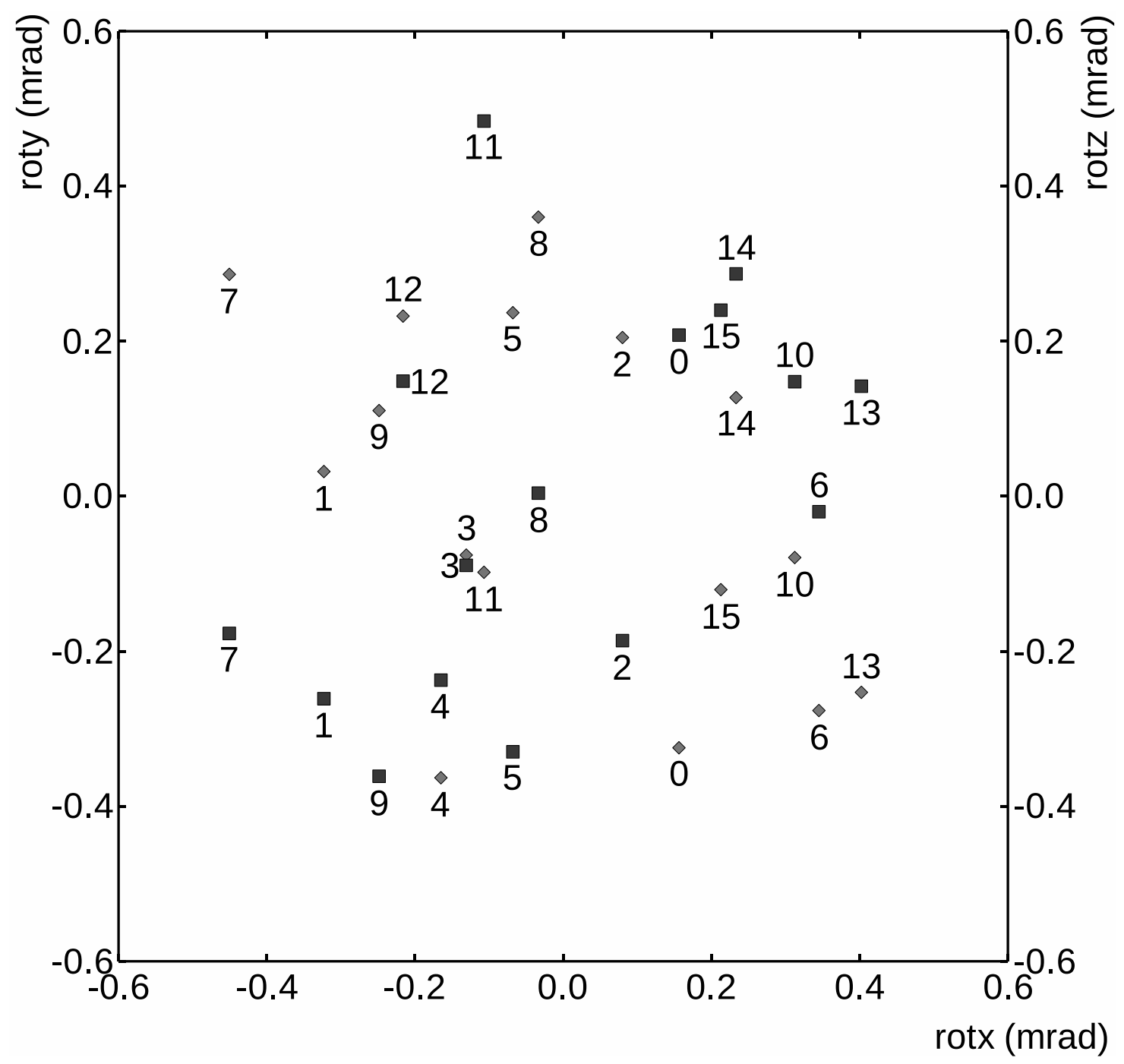

Figure 31: Correlation between the different rotation angles of each tower (the number of each tower is denoted in the diagram). Squares denote the correlation between $\operatorname{rot} x$ ( $x$-axis) and roty (left $y$-axis label), and diamonds between $\operatorname{rot} x$ and $\operatorname{rot} z$ (right $y$-axis label).

The alignment of the TKR has been checked twice before launch (since October 2006) and four times after launch (since June 2008). No significant changes of the positions and rotations were observed. We expect updates to be made annually.

\subsection{LAT alignment with respect to the spacecraft}

The LAT reconstructs the direction of each photon in the LAT reference system. To convert this to celestial coordinates, we require the celestial orientation of the LAT. The spacecraft orientation is provided by the Guidance, Navigation and Control system, or star-tracker. This system involves optical telescopes on an optical bench, and a star field pattern recognition system, which was calibrated before launch. The LAT system is nominally the same as the spacecraft system, but small deviations are expected due to uncertainty in the ground 
alignment process, thermal variations, launch vibrations, relaxation in $0 \mathrm{~g}$, or the inter-tower alignment process. Measuring these deviations, by comparing the LAT view of the sky with that seen by the star-tracker is called "boresight alignment".

The boresight alignment is determined via likelihood maximization for gamma rays near bright, identified celestial point sources of gamma-ray emission. The likelihood analysis is "binned" in the sense that the gamma rays are not considered individually but binned into maps in celestial coordinates. Separate maps are used for different energy ranges because the point-spread function (PSF) depends strongly on energy. The likelihood is defined in terms of the Point Spread Function (PSF), the background (sum of celestial sources and residual charged particle bacgrounds, estimated independently for each source), and the boresight alignment angles. We measure the boresight alignment by maximizing the total binned likelihood of the point source model (background is included) with respect to three angles characterizing an arbitrary rotation. We chose the convention of performing consecutive rotations about the $x, y$, and $z$ axes. These are defined by the spacecraft: $z$ is along the symmetry axis (pointing direction), and $y$ is along the solar panels. Since the angles are small, less than a degree, the order of the rotations is irrelevant. Rotations about the $x$ and $y$ axes thus correspond to angular deviations of the same size, while the rotation about the $z$ axis affects only tracks that traverse the detector at off-axis angles. The procedure for calculating the likelihood transforms both the source position (i.e. celestial coordinates) and the photon position into spacecraft coordinates, rotates the photon's position, and determines the likelihood that the photon is consistent with the source location. Details of the likelihood method are discussed elsewhere [29].

We first define a set of reference bright point sources using the likelihood itself to select the most significant. We select photons within the $99 \%$ PSF containment of the sources. Since the angular containment is energy dependent, photons are divided into energy bands, using an average PSF for each band to define the expected deviation distribution. The PSF is non-Gaussian, and has been found to be well modeled by the following power-law expression [11],

$$
\operatorname{PSF}\left(\delta, E_{i}\right)=\left(1-\frac{1}{\gamma\left(E_{i}\right)}\right)\left(1+\frac{\delta^{2}}{2 \sigma\left(E_{i}\right)^{2} \gamma\left(E_{i}\right)}\right)^{-\gamma\left(E_{i}\right)},
$$

where $E_{i}$ is the energy bin of the photon, $\delta$ is the angular separation between reconstructed and true direction, and $\gamma\left(E_{i}\right)$ and $\sigma\left(E_{i}\right)$ are energy dependent parameters. The parameter $\gamma\left(E_{i}\right)$ determines the tails of the distribution and at low energues has a value of $\sim 2.25$, which decreases at high energies, creating longer PSF tails. The parameter $\sigma\left(E_{i}\right)$ has a power-law dependence at lower energies and reaches an asymptotic value at high energies determined by the silicon detector readout pitch. We compute $\delta$ for all spatial bins and weight the likelihood by the number of counts per bin. The parameters of the PSF were estimated by extensive Monte Carlo simulations, but have been since refined with on-orbit data. We select photons from energy bands above $500 \mathrm{MeV}$, which contain little or no background. We calculate a likelihood for each source by modeling it as point source in a uniform background. Treating the remaining background on the angular scale of the PSF as uniform is a good approximation, and would be significant only in the tails where the contribution to the likelihood is small. 
The bright point sources for daily alignment check are selected based on the Test Statistic (TS), which is twice the difference of the log likelihood of a point source in a uniform background and the log likelihood of a purely uniform background for a particular energy band. The total TS is then the sum of the TS's for the energy bands above $500 \mathrm{MeV}$. Since the background fraction is already small, the assumption of uniformity is a good approximation to the distribution of the background events, and we select all sources with a total TS greater than 25. For the example shown in Figure 32, 6 sources match this criterion, and these are used as seeds for the alignment procedure. The largest contribution comes from the Vela pulsar, which is dominated by photons between $1 \mathrm{GeV}$ and its $10 \mathrm{GeV}$ cutoff.

The likelihood is maximized with respect to the background for each energy band, since the shape of the PSF depends on energy. The total likelihood for a point source is then the product of the likelihood in each energy band.

Figure 32 shows typical projected error ellipses resulting from the likelihood fit. The crosses correspond to the ideal location and the dashed and solid contour lines represent 1 and 2 sigma contours derived from the log likelihood, respectively. A typical day in orbit yields a precision of 1.5 arcmin in $x$ and $y$, and 2.4 arcmin in $z$, when using 174 photons collected from six astronomical sources. We expect, after the analysis of the first year data to reach the required value of 4 arcsec for the boresight alignment residuals.

As shown in Figure 33 we make independent measurements of the boresight alignment for each week, to monitor the stability. The figure shows the cumulative mean (dash-dotted line) for the rotation angles about the $x, y$ and $z$ axes for a period of 3 months. The cumulative mean is just the average of the parameters weighted by the errors. We use the same sources and data to optimize the combined likelihood with respect to the PSF parameters themselves, and feed this back into the analysis. Weekly measurements indicate a stability of 0.3 arcmin, constant with the statistical errors.

We repeated the likelihood fits for a period of about 5 months. The results for the rotation angles are shown in Figure 34. We clearly see the improvement with more statistics. As expected, rotations about $x$ and $y$ axis lead to similar results (6 arcsec), while for rotations around $z$ we obtain values approaching 8 arcsec. These values should be added in quadrature to other contributions to obtain the systematic uncertainties for localizing point sources.

\section{Conclusions}

We have discussed the on-orbit calibrations for the Fermi LAT, which include synchronization of trigger signals, optimization of delays for latching data, determination of detector thresholds, gains and responses, evaluation of the perimeter of the South Atlantic Anomaly (SAA), measurements of live time and of absolute time and internal and spacecraft boresight alignments. The results summarized in Table 10 were obtained using known astrophysical sources, galactic cosmic rays, and charge injection. There were only minor changes to calibration constants since launch and these quantities have been stable during the first eight months of operations.

The LAT with almost a million channels is a remarkably stable instrument and is expected to operate during the next few years with dedicated calibration runs of about three hours every three months. The frequency with which calibration updates are expected to 

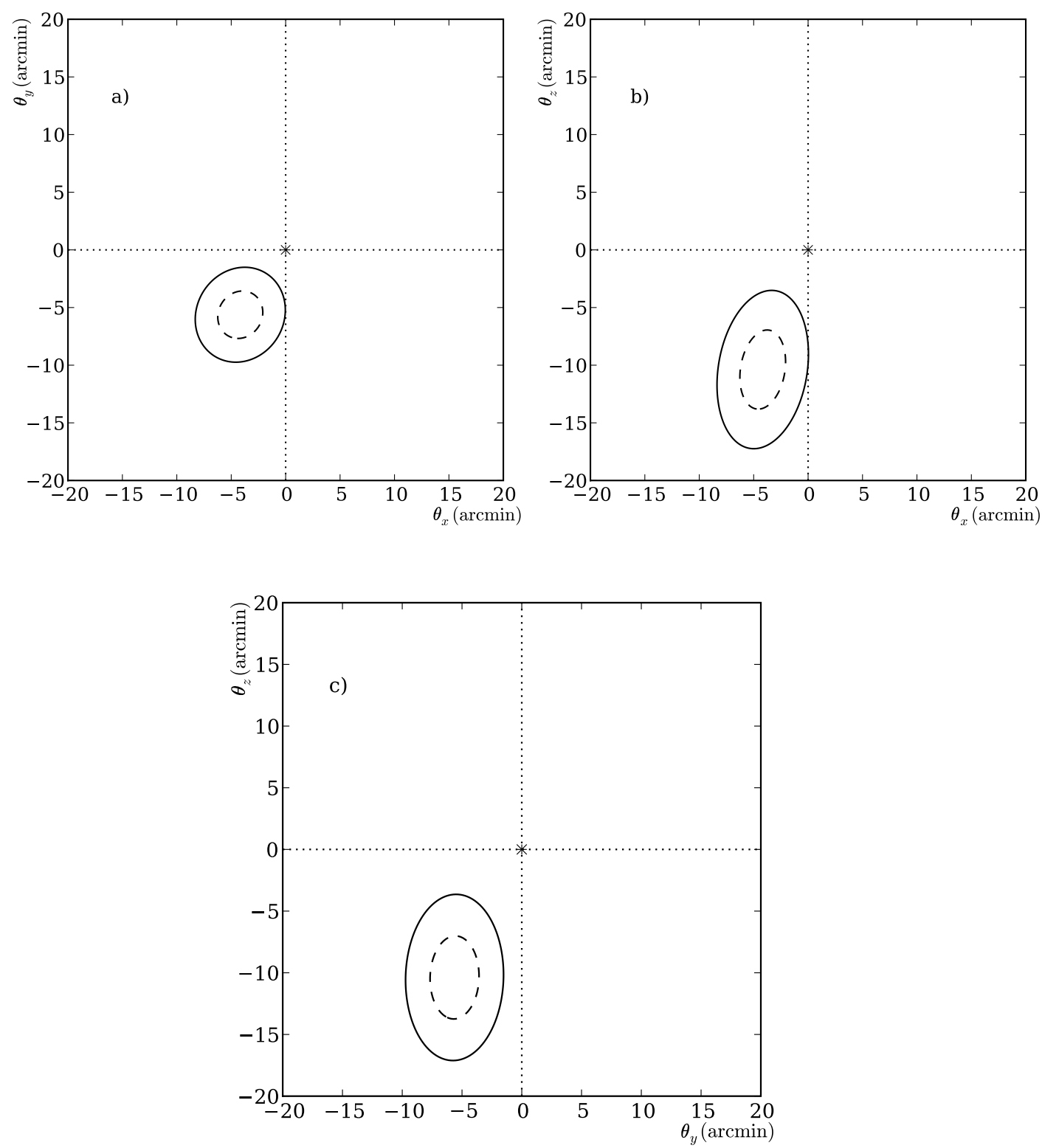

Figure 32: Projection of the likelihood surfaces into each of the planes a) $y x, b) z x$ and c) $z y$ for one day of data (August 3, 2008). Crosses correspond to the ideal location (perfect alignment), and contours are 68\% (dashed) and 95\% (solid) containment. 

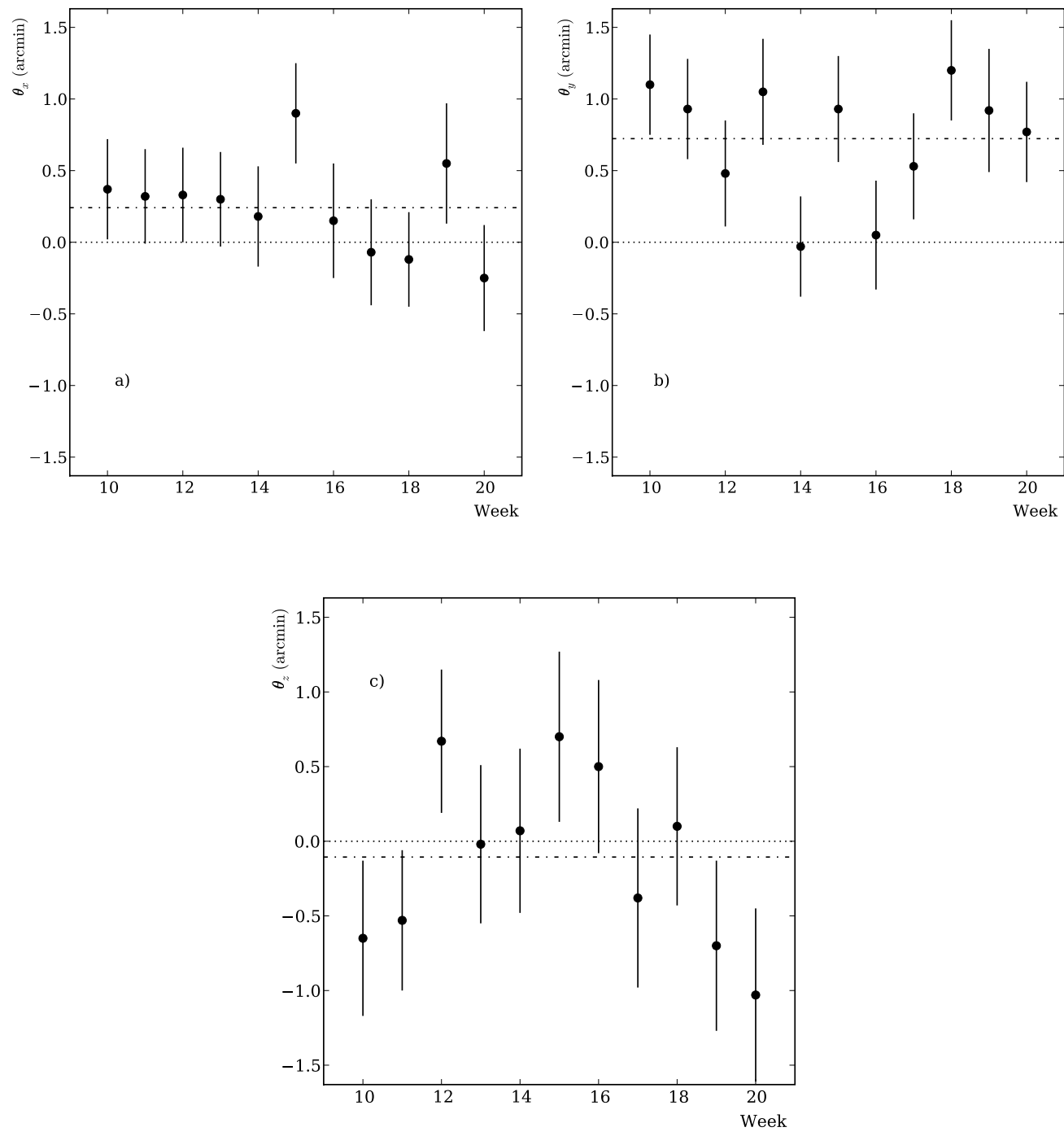

Figure 33: Results of the likelihood fit for weekly measurements over a period of 3 months for the rotation angles about the a) $x$, b) $y$ and c) $z$ axes. The cumulative mean is displayed as dash-dotted line, while the dashed line shows the reference at zero $(y$ axis scales are different). The start date for these measurements on Mission week 10 corresponds to August $10,2008$. 


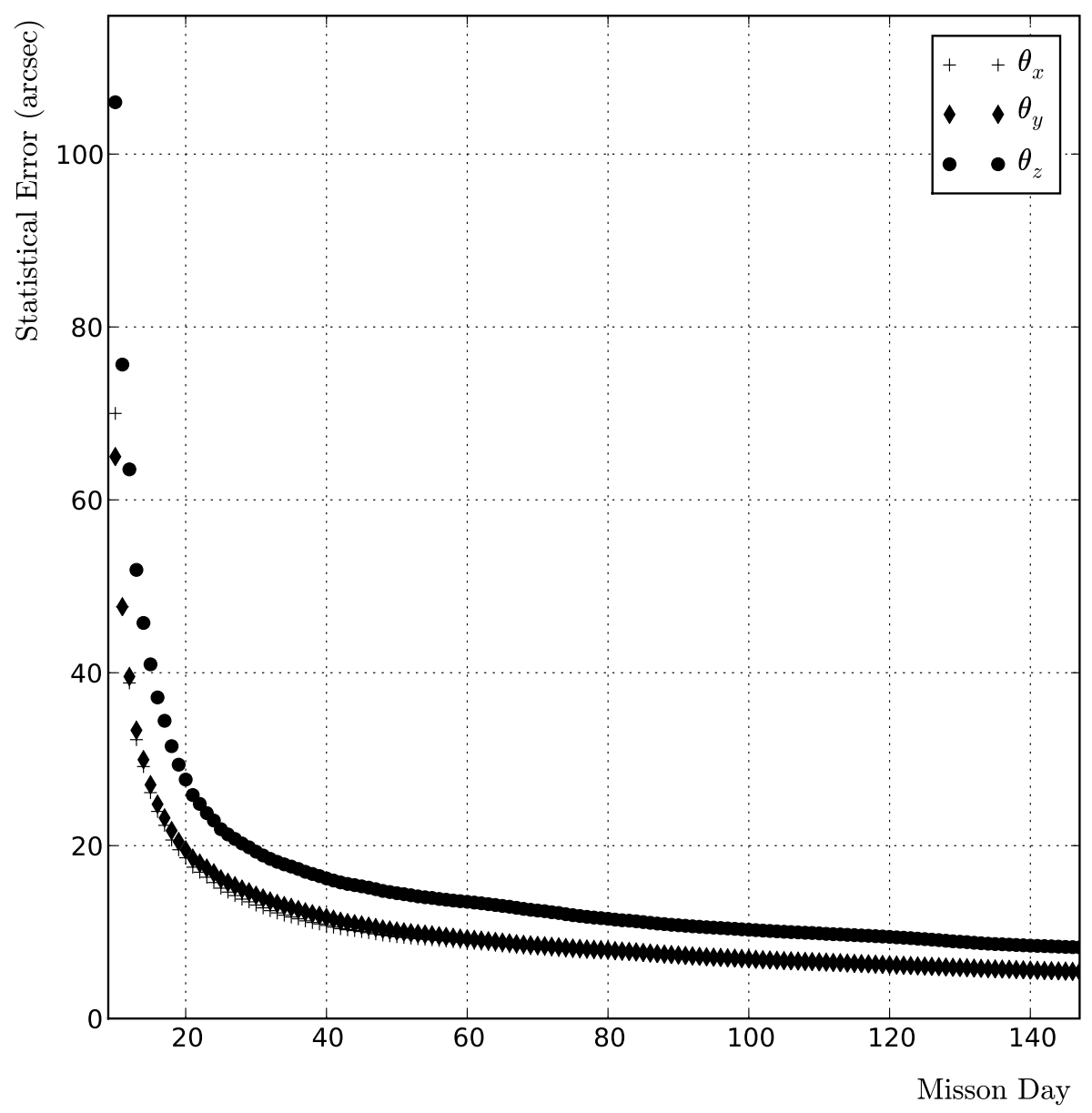

Figure 34: Data correspond to results of the likelihood fit for measurements over a period of 5 months for the rotation angles about the a) $x$, b) $y$ and c) $z$ axes. 
occur varies from three to twelve months, but these do not necessarily impact the LAT performance in any significant way. As a consequence, changes to high level datasets due to these calibrations will be infrequent. The results reported here have been used to calibrate the LAT datasets to be publicly released in August 2009.

\section{Acknowledgments}

The Fermi LAT Collaboration acknowledges the generous ongoing support of a number of agencies and institutes that have supported both the development and the operation of the LAT as well as scientific data analysis. These include the National Aeronautics and Space Administration and the Department of Energy in the United States, the Commissariat à l'Energie Atomique and the Centre National de la Recherche Scientifique / Institut National de Physique Nucléaire et de Physique des Particules in France, the Agenzia Spaziale Italiana and the Istituto Nazionale di Fisica Nucleare in Italy and the Istituto Nazionale di Astrofisica, the Ministry of Education, Culture, Sports, Science and Technology (MEXT), High Energy Accelerator Research Organization (KEK) and Japan Aerospace Exploration Agency (JAXA) in Japan, and the K. A. Wallenberg Foundation and the Swedish National Space Board in Sweden.

Additional support for science analysis during the operations phase from the following agencies is also gratefully acknowledged: the Istituto Nazionale di Astrofisica in Italy and the K. A. Wallenberg Foundation in Sweden for providing a grant in support of a Royal Swedish Academy of Sciences Research fellowship for JC.

\section{References}

[1] W. B. Atwood et al., in press Astrophysical Journal.

[2] C. Meegan et al, in preparation.

[3] D.J. Thompson et al., ApJ. Suppl. Vol 86 (1993) 629.

[4] W. B. Atwood et al., Astropart. Phys. 28, (2007) 422.

[5] T. Ohsugi et al, Nucl. Inst. and Meth. A 541 (2005) 29.

[6] R. P. Johnson, P. Poplevin, H. F.-W. Sadrozinski, and E. N. Spencer, IEEE Trans. Nucl. Sci. 45, (1998) 927.

[7] L. Baldini et al., IEEE Trans. Nucl. Sci. 53, (2006) 466.

[8] J. E. Grove et al., in preparation.

[9] A. Moiseev et al., Astropart. Phys. 27, (2007) 339.

[10] A. Moiseev et al., Nucl. Inst. and Meth. A 583, (2007) 372.

[11] A. Abdo et al., in preparation. 


\begin{tabular}{|c|c|c|c|}
\hline Category & Title & Summary & Sec. \\
\hline Trigger & Time coincidence window & $700 \mathrm{~ns}$, settings within $50 \mathrm{~ns}$ & 3.2 \\
\hline Trigger & Fast trigger delays & synchronization within $50 \mathrm{~ns}$ & 3.2 \\
\hline Trigger & Delays for latching data & optimized within $50 \mathrm{~ns}$ & 3.2 \\
\hline $\mathrm{ACD}$ & Pedestal & width $<4$ ADC bins or $<0.01$ MIPs & 4.1 \\
\hline ACD & Coherent noise & removed down to 0.005 MIP & 4.1 \\
\hline ACD & MIP peak & stability $<10 \%$ & 4.2 \\
\hline $\mathrm{ACD}$ & High range $(\mathrm{CNO})$ & width of carbon peak $\sim 20 \%$ of peak & 4.2 \\
\hline $\mathrm{ACD}$ & Veto threshold & turn-on at $0.4-0.5$ MIP, set within \pm 0.01 MIP & 4.3 \\
\hline $\mathrm{ACD}$ & High level discriminator & turn-on at 24-26 MIP, set within \pm 1 MIP & 4.3 \\
\hline CAL & Pedestal & RMS within $0.1-0.2 \mathrm{MeV}$ & 5.1 \\
\hline CAL & Electronics linearity & corrected to $\leq 1 \%$ of the measured energy & 5.2 \\
\hline CAL & Energy scales & $\begin{array}{c}\text { spread crystal-to-crystal } \leq 1 \% \\
\text { stability of peaks: } \operatorname{MIP}(\leq 2 \%), \text { carbon }(\leq 1 \%)\end{array}$ & 5.2 \\
\hline $\mathrm{CAL}$ & Light asymmetry & $2 \mathrm{~mm}$ (LEX1), $9 \mathrm{~mm}$ (HEX8) from 200-900 $\mathrm{MeV}$ & 5.3 \\
\hline CAL & Zero-suppression threshold & set at $2 \mathrm{MeV}, \sim 10 \mathrm{x}$ electronics noise & 5.4 \\
\hline CAL & Low-energy threshold & set at $100 \mathrm{MeV}( \pm 1 \%)$ & 5.4 \\
\hline CAL & High-energy threshold & set at $1 \mathrm{GeV}( \pm 2 \%)$ & 5.4 \\
\hline CAL & Upper level discriminators & set at $5 \%$ below saturation level & 5.4 \\
\hline TKR & Noisy channels & $\begin{array}{l}\text { avg strip occ. }\left(10^{-5}\right) \text {, add } \leq 10 \% \text { to the TKR data } \\
\text { electronic noise occupancy }\left(10^{-7}\right), 0.04 \% \text { disabled }\end{array}$ & 6.1 \\
\hline TKR & Trigger threshold & $\begin{array}{l}\text { set at } \sim 0.28 \text { MIP, spread channel-to-channel } \sim 5 \% \\
\leq 1 \text { ADC shift since launch ( } 99.96 \% \text { of channels) }\end{array}$ & 6.2 \\
\hline TKR & Data latching threshold & spread channel-to-channel 12\% & 6.2 \\
\hline TKR & ToT conversion parameters & fitted to $\sim 8 \%$ (statistical error) & 6.3 \\
\hline TKR & MIP scale & RMS of correction factor $(9 \%)$ & 6.4 \\
\hline SAA & SAA polygon & in SAA for $\sim 13 \%$ of the orbit time & 7 \\
\hline Timing & LAT timestamps & $<0.3 \mu$ s with respect to a reference GPS & 9 \\
\hline Alignment & Intra tower & for details see Tables 7 and 9 & 10.1 \\
\hline Alignment & Inter tower & for details see Tables 8 and 9 & 10.2 \\
\hline Alignment & LAT boresight & $\theta_{x}, \theta_{y} \leq 6^{\prime \prime}$ and $\theta_{z} \leq 8^{\prime \prime}$ (5 months) & 10.3 \\
\hline
\end{tabular}

Table 10: Summary of the on-orbit Fermi LAT calibrations. 
[12] W. Atwood et al., Nucl. Inst. and Meth. A 446 (2000) 444.

[13] E. do Couto e Silva et al., Nucl. Inst. and Meth. A 474, (2001) 19.

[14] A. Abdo et al., in preparation.

[15] A. Abdo et al., in preparation.

[16] A. Moiseev et al., Astropart. Phys. 22, (2004) 275.

[17] B. Lott et al., Nucl. Inst. and Meth. 560, (2006) 395.

[18] D. M. Sawyer and J. I. Vette, AP-8 Trapped Proton Environment for Solar Maximum and Solar Minimum, NSSDC/WDC-A-R\&S 76-06, 1976.

[19] D. Heynderickx, M. Kruglanski, V. Pierrard, J. Lemaire, M. D. Looper, and J. B. Blake, IEEE Trans. Nucl. Sci., 46, (1999) 1475.

[20] IGRF-10, IAGA Division V-MOD Geomagnetic Field Modeling, http://www.ngdc.noaa.gov/IAGA/vmod/igrf.html

[21] M. Casolino, et al. Observations of primary, trapped and quasi-trapped particles with the PAMELA experiment. to appear in the proceedings of the $30^{\text {th }}$ International Cosmic Ray Conference (ICRC 2007) 3-11 Jul 2007, Merida, Yucatan, Mexico.

[22] A. Abdo et al submitted to Science (2008).

[23] D. A. Smith et al. A\&A 492 (2008) 923.

[24] M. de Naurois, J. Holder et al. ApJ 566, (2002) 343.

[25] A. Abdo et al., in press Astrophysical Journal (2008).

[26] Global Postioning System Standard Positioning Service Performance Standard', 4th edition (September 2008), http://pnt.gov/public/docs/2008-SPSPS.pdf, p. A-16, section A.4.8

[27] A. Abdo et al., submitted to ApJ.

[28] A. Abdo et al., in preparation.

[29] A. Abdo et al., in preparation. 